\title{
A semi-microscopic model of synaptic transmission and plasticity
}

\author{
Dissertation \\ zur Erlangung des Doktorgrades \\ der Mathematisch-Naturwissenschaftlichen Fakultäten \\ der Georg-August-Universität zu Göttingen
}

vorgelegt von

Julia Trommershäuser

aus Frankfurt am Main

Göttingen, den 13. März 2000 
Referentin: Prof. Dr. A. Zippelius

Korreferent: Prof. Dr. T. Geisel

Tag der mündlichen Prüfung: 26. April 2000 


\section{Contents}

1. Introduction 3

1.1. Synaptic transmission in the brain . . . . . . . . . . . . . . . 3

1.2. Glutamatergic synapses . . . . . . . . . . . . . . . . . . 5

1.3. Theoretical approaches to synaptic transmission . . . . . . . . . . . . 6

1.4. Overview and objectives of this work . . . . . . . . . . . . . . . . . 9 9

\begin{tabular}{ll}
\hline 2. Presynaptic vesicle dynamics & 11
\end{tabular}

2.1. Two-Pool-model of vesicle recruitment . . . . . . . . . . . . . . . . . . . . 13

2.2. Resting conditions and release following a single action potential . . . . . . 16

2.3. Facilitation of release-probability . . . . . . . . . . . . . . . . 17

2.3.1. Buffered calcium diftusion and tacilitation of release . . . . . . . . . 17

2.3.2. Calcium-binding site model of facilitation . . . . . . . . . . . . . . 20

2.4. Calculating EPSCs evoked by a sequence of action potentials . . . . . . . . 22

2.5. Results . . . . . . . . . . . . . . . . . . . . . . . . 25

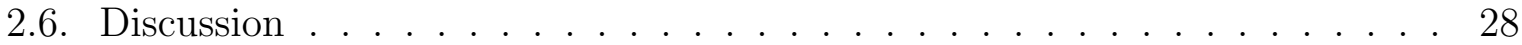

3. Transmitter dynamics within the synaptic cleft 33

3.1. Diffusion of transmitter in the synaptic cleft . . . . . . . . . . . . . . . . 34

3.2. Two-dimensional model of the synaptic cleft . . . . . . . . . . . . . . 36

3.3. Estimate of the diffusion coefficient . . . . . . . . . . . . . . . . 37

3.4. Outlook: Postsynaptic currents yield information about the transmitter time course in the cleft . . . . . . . . . . . . . . 39

\begin{tabular}{ll}
\hline 4. Studying the postsynaptic side & 41
\end{tabular}

$4.1 . \quad$ Receptor kinetics . . . . . . . . . . . . . . . . . . . . . . . . 43

4.1.1. Kinetic seven-state model of AMPA receptors . . . . . . . . . . . . 44 
4.1.2. Kinetic three-state model of AMPA receptors . . . . . . . . . . . 45

4.1.3. Fit to experimental data: insight from outside-out patch recordings . . . . . . . . . . . 45

4.1.4. Non-stationary fluctuation analysis . . . . . . . . . . . . . . . 46

4.1.5. Estimate of kinetic rate constants from experiments . . . . . . . . . 47

4.2. Monte Carlo simulations of miniature EPSC . . . . . . . . . . . . . . 49

4.3. Receptor dynamics calculated by local chemical kinetic equations . . . . . 52

4.4. Correspondence of kinetic and Monte Carlo model . . . . . . . . . . . . . . 55

4.5. Results for hippocampal synapses . . . . . . . . . . . . . . . . 56

4.6. Results for brainstem interneurons . . . . . . . . . . . . . . 65

4.7. Discussion . . . . . . . . . . . . . . . . . . . . 73

5. Transmission properties of a single synaptic connection 75

5.1 . Model and Methods . . . . . . . . . . . . . . . . . . . 77

5.2. Synaptic transmission of information . . . . . . . . . . . . . . 82

5.3. Results . . . . . . . . . . . . . . . . . . . . . . . . . . . 83

5.4 Discussion . . . . . . . . . . . . . . . . . . . . . . . . . . . . . . 88

6. Conclusion and outlook 91

A. Release-sites, calcium microdomains and global residual calcium 95

A.1. Concentration profile due to a single conducting calcium channel . . . . . . 95

A.2. Calcium concentration at the release-site . . . . . . . . . . . . . . . 98 


\section{Introduction}

\subsection{Synaptic transmission in the brain}

The synapse is the point of functional contact between one neuron and another. It is the primary place at which information is transmitted from neuron to neuron in the central nervous system (CNS). Most inter-neural communication relies on the use of a chemical intermediary, or neurotransmitter, which is secreted following an action potential (AP) by presynaptic cells to influence the activity of postsynaptic cells. These sites of functional contact are called chemical synapses.

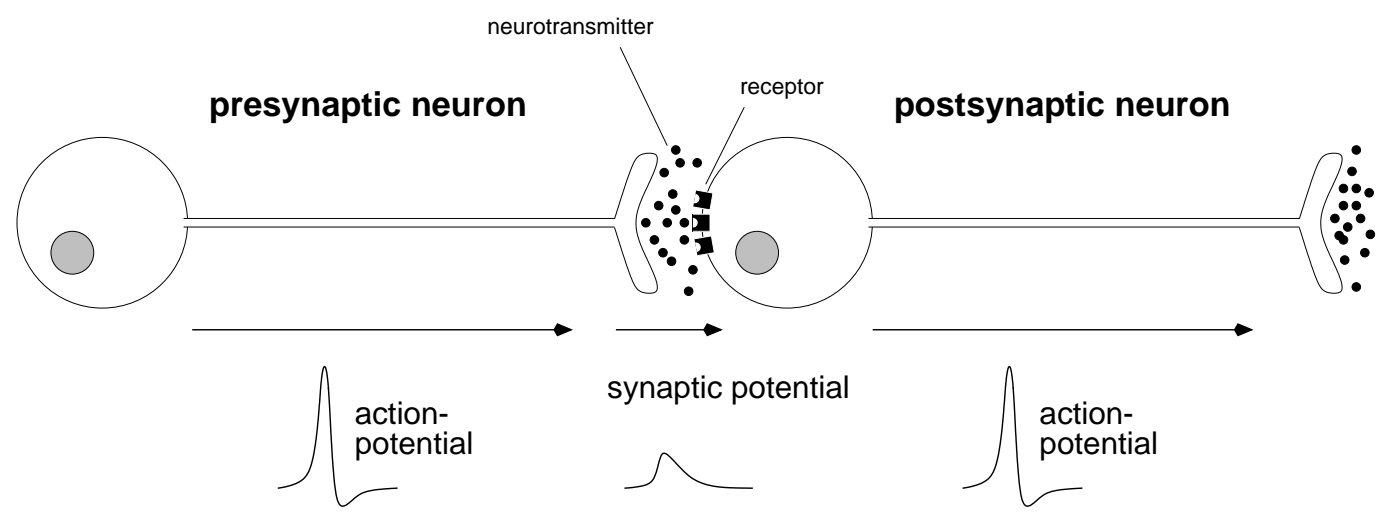

Figure 1.1.: Transmission of information between neurons by chemical and electrical signals. Following an electrical signal neurotransmitter is secreted from the presynaptic nerve terminal of a chemical synapse. The neurotransmitter binds to postsynaptic receptors to mediate flux of ions across the membrane, driving the membrane potential away from resting value and causing a so called postsynaptic potential. Synaptic potentials may trigger an action potential in the cell body, which runs down the axon to be conveyed from the nerve terminal onto the next cell (from Hall, 1992).

At each chemical synapse a region of membrane in the presynaptic cell that is specialized for rapid secretion is closely and firmly attached to a particular region on the postsynaptic cell containing a high density of receptors for the neurotransmitter. In this way, chemical communication in the CNS is specifically directed from one cell to another. Electrical 
signals in the presynaptic cell cause the release of neurotransmitter; its binding to surface receptors triggers an influx of ions into the postsynaptic cell causing the synaptic potential (see Fig. 1.1), i.e. a shift of the postsynaptic membrane potential away from resting value.

The number of synaptic connections a neuron forms can be extraordinarily large and many afferents can interact and influence a postsynaptic neuron, by either excitatory or inhibitory effects, depending on the ions that permeate the channels on the postsynaptic side operated by the receptor. The resulting responses are either excitatory postsynaptic potentials (EPSPs) or inhibitory postsynaptic potentials (IPSPs), depending on whether they drive the cell towards a point above or below its firing threshold.

Most central neurons exhibit a clearly defined cell body, called soma, from which neurites arise. In most cases these neurites can be divided into a single axon, which may branch into a few or many collaterals at some distance from the cell and in an often elaborated, highly branched network of dendrites. In general, axons form the presynaptic elements enlarging into a single axon terminal at the end of each collateral. The postsynaptic element is most commonly the dendritic spine, the shaft of the dendrite, or the cell soma.

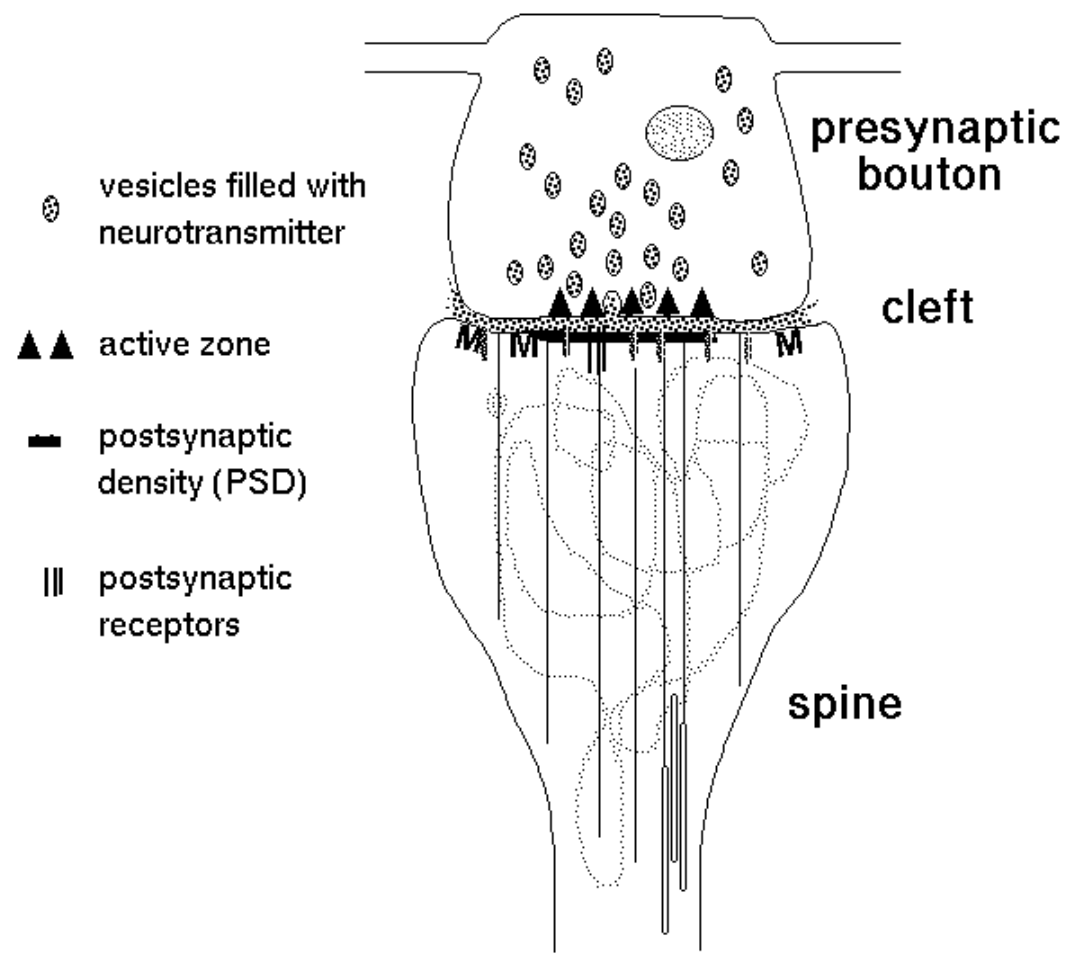

Figure 1.2.: Schematic picture of a simple spine synapse. Pre- and postsynaptic part are separated by a very narrow synaptic cleft. The presynaptic bouton contains vesicles, some of which are docked at the active zone. The postsynaptic density opposite the active zone contains postsynaptic receptors (from Edwards, 1995a). 


\subsection{Glutamatergic synapses}

The major part of excitatory contacts between neurons in the cortex release glutamate as their transmitter. In most parts of the work presented here we will focus on the common form of glutamatergic synapse displayed in Fig. 1.2, which is formed between axons and dendritic spines and is found throughout the cortex, on hippocampal CA1 and granule cells, on cerebellar Purkinje cells, interneurons in the brainstem and in many other areas of the brain. This synapse consists of three basic elements (Fig. 1.2): First, the axonal enlargement forming the presynaptic terminal, which we also refer to as presynaptic bouton, second, the gap between the pre- and postsynaptic part, the synaptic cleft, and third, the small extension from the dendrite of the postsynaptic cell, the dendritic spine, which contains the postsynaptic receptors.

Upon arrival of an AP voltage-gated calcium channels, which are strategically colocalized with the synaptic vesicles docked within the active zone (= readily-releasable vesicles), give way for calcium entering the postsynaptic terminal. The local intense rise in $\mathrm{Ca}^{2+}$ concentration triggers the fusion of docked vesicles with the cell membrane and the release of their contents in the synaptic cleft, which is approximately $15 \mathrm{~nm}$ wide (Edwards, 1995b). The fusion of one vesicle releases about 1000 to 4000 transmitter molecules in less than a millisecond, which activate glutamatergic postsynaptic receptors that mediate the current (= excitatory postsynaptic current, EPSC) recorded on the postsynaptic side (Clements, 1996; Edwards, 1995b). After release transmitter molecules are rapidly removed from the cleft by diffusion or binding to glutamate transporters which ensure glial and neuronal uptake and a rapid clearance of abundant transmitter molecules from the cleft and its vicinity (Diamond and Jahr, 1997; Dzubay and Jahr, 1999; Wang et al., 1998; Rusakov and Kullmann, 1998; Kullmann and Asztely, 1998).

The release of glutamate activates two different types of ligand-gated ion channels, first AMPA ( $\alpha$-amino-3-hydroxy-5-methylisoxazole-4-propionic acid)/kainate receptors and, second, NMDA ( $N$-methyl-D-aspartate) receptors. AMPA receptors activate quickly in less than a millisecond and mediate the major contribution of glutamate activated excitatory transmission. The activation of NMDA receptors occurs on a much slower time-scale of several milliseconds. Around resting potential of $-70 \mathrm{mV}$ NMDA receptors are nonconducting due to a voltage dependent magnesium block and activate after membrane depolarization (Kaczmarek et al., 1997). The number of AMPA receptors within a glutamatergic synapse has been estimated as 30 to 100 receptors, whereas there are only few, i.e. less than ten NMDA receptors (Edwards, 1995b; Spruston et al., 1995). The latter hardly contribute to fast excitatory transmission and phenomena of short-term plasticity, but may be important for development and long-term changes in synaptic efficacy (Castro-Alamancos and Connors, 1997; Kaczmarek et al., 1997; Markram et al., 1998b).

Although a variety of electrophysiological and anatomical studies have been performed on glutamatergic synapses (Edwards, 1995a; Walmsley et al., 1998, for reviews), essential steps of the transmission process are not understood in detail. This has inspired several attempts to gain further insight in the primary determinants of synaptic transmission on 
the basis of theoretical models. In the following section we will briefly summarize previous theoretical work before giving an outline of our approach chosen in the present work.

\subsection{Theoretical approaches to synaptic transmission}

Approaches to model synaptic transmission usually follow two different lines: first, phenomenological approaches trying to comprise the essentials of synaptic transmission by a few model parameters which do not necessarily need a physiological or biophysical counterpart in the "real" cell. The aim of these models is a complete characterization of certain aspects of synaptic transmission, e.g. the average synaptic activity for a given presynaptic stimulus pattern. These models use a minimal number of parameters to allow a quantitative description and prediction of changes in synaptic efficacy and aim for a general applicability to a variety of experimental data. Phenomenological models of dynamic synapses as well as the analysis of distributions of EPSC amplitudes by quantal analysis belong in this category of modeling approaches.

Second, a different strategy of modeling is based on electrophysiological experiments and intends to formulate kinetic models of the underlying physiological mechanisms in order to reproduce experimentally observed responses and time scales. These models attempt to be closely tied to underlying physiological mechanisms. For instance, kinetic schemes are used to model the dynamics of presynaptic release and form the theoretical fundament of Monte Carlo simulations of transmitter diffusion and receptor kinetics.

\section{Phenomenological models of dynamic synapses}

A phenomenological model of synaptic transmission has been designed in oder to study synaptic transmission between pairs of neurons (Tsodyks and Markram, 1997; Varela et al., 1997; Zador and Dobrunz, 1997). Without specifying the underlying physiological mechanisms it is assumed that a synapse is characterized by a finite amount of resources. These resources occur in the three states "effective", "inactive", and "recovered". Each AP activates a fraction of the resources which are available in the recovered state and subsequently quickly inactivate, from where they return on a much slower time-scale into the recovered state. In addition facilitation of release as experimentally observed after repetitive presynaptic stimulation is implemented by increasing the amount of resources activated per AP.

After fit of the model to experiments this theoretical approach yields the synaptic activity as function of input-frequency. It manages to reproduce averaged experimentally recorded synaptic responses between pyramidal neurons (Abbott et al., 1997; Tsodyks and Markram, 1997). The model has been implemented in larger models of neural networks and shown to generate complex patterns of regular and irregular regimes of network activity (Tsodyks et al., 1998). It computes properties of synaptic transmission as function of "limited synaptic resources". The model, however, does not allow to differentiate between 
pre- or postsynaptic processes contributing to synaptic transmission. Furthermore, only average synaptic responses as generated by taking the mean over many measurements are calculated, whereas the experimental outcome of individual measurements is usually very noisy.

\section{Quantal analysis}

The noise in synaptic responses can be seen in broad distributions of amplitudes of synaptic currents. Quantal analysis is a method used for analyzing these distributions and for infering functional significance from their shape. It is based on the idea that transmitter is packaged in individual vesicles and that synaptic currents are due to the stochastic release of individual vesicles of transmitter into the synaptic cleft (del Castillo and Katz, 1954; Boyd and Martin, 1956). It is assumed that a number of $n$ quanta of transmitter is available in the presynaptic terminal to be released, and every quantum gives roughly the same electrical signal in the postsynaptic cell. This is the quantal amplitude $Q$, which sums linearly with all other quanta released. If $p$ denotes the average release-probability for the release of a single quantum, the relative probability of observing $0,1, \ldots, n$ quanta released is then given by a binomial distribution.

This binomial function can be fitted to distributions of EPSC amplitudes to extract the three parameters $Q, p$, and $n$. The quantal size $Q$ represents the mean amplitude of the miniature distribution, 1 or the first peak in the distribution of evoked postsynaptic currents. The release-probability of individual vesicles $p$ is given by the relative height of the peaks and the number of (readily-releasable) vesicles $n$ by the total number of peaks in a distribution of indefinite sample size. Quantal analysis has not only been used to characterize properties of synaptic connections by estimating $Q, n$ and $p$ (Bekkers and Stevens, 1995; Edwards et al., 1990; Frerking et al., 1995; Hessler et al., 1993; Jonas et al., 1993; Rosenmund et al., 1993, for instance), but also to describe short- and long-term changes in synaptic efficacy (Ambros-Ingerson and Lynch, 1993; Bekkers and Stevens, 1990; Foster and McNaughton, 1991; Kullmann and Nicoll, 1992; Malinow and Tsien, $1990)$.

Originally designed for the analysis of synaptic responses at the neuromuscular junction (del Castillo and Katz, 1954; Boyd and Martin, 1956), the transfer of quantal analysis to central synaptic transmission requires some caution (see Edwards, 1995a and 1 for a detailed discussion): First, the quantal size as determined by the first peak in amplitude distributions is very small and often strongly fluctuating. This suggests that postsynaptic receptors are saturated by the release of a single vesicle and fluctuations in quantal size are due to variations in the receptor number. Additionally the assumptions of quantal analysis concerning the uniformity, independency and synchrony of vesicle release often appear problematic.

\footnotetext{
${ }^{1}$ Miniature synaptic currents occur in the absence of APs and are thought to be due to the release of the contents of individual vesicles.
} 


\section{Kinetic models}

Kinetic models which intend to incorporate specific properties of the underlying physiological mechanisms have been formulated either for presynaptic processes of vesicle recruitment and release or for the diffusion and interaction of neurotransmitter with the postsynaptic receptor population.

\section{Kinetic models of presynaptic vesicle dynamics}

The amount of presynaptic vesicles released for a given stimulus protocol can be theoretically modeled by kinetic rate equations. The idea is that upon stimulation presynaptic vesicles are released and the number of vesicles docked within the active zone, the so called pool of readily-releasable vesicles, is depleted. The readily-releasable pool is slowly replenished within a few seconds, a process that first has been been modeled by a kinetic two-state model, comprising a filled and empty release-site (Liley and North, 1953). Subsequently the comparison of theory and experiments has lead to modifications of this simple depletion model by suggesting activity-dependent recruitment rates (Fisher et al., 1997; Dittmann et al., 2000; Zucker, 1999).

\section{Analytical and Monte Carlo models of transmitter diffusion and postsynaptic receptor kinetics}

In close connection to electrophysiological experiments analytical and stochastic models of transmitter diffusion and receptor kinetics have been developed. Analytical descriptions of the diffusion of neurotransmitter in the cleft are coupled to kinetic models of receptor kinetics and are solved analytically (Eccles and Jaeger, 1958; Kleinle et al., 1996; Uteshev and Pennefather, 1996a) or numerically (Land et al., 1981; Holmes, 1995).

Monte Carlo simulations are performed to capture spatial and stochastic effects of neurotransmitter diffusion as well as the intrinsic noise of the receptors dynamics. Following the work of Bartol et al. (1991) transmitter motion is modeled by a random walk for individual transmitter molecules. The stochasticity of receptor kinetics is included by simulating individual receptors on the basis of their corresponding kinetic schemes for channel opening (see Sec. 4.2 for details and references). For a given set of synaptic parameters Monte Carlo simulations yield distributions of postsynaptic responses which can be compared to experiments on miniature EPSCs, i.e. postsynaptic currents caused by the release of a single vesicle, and are used to study transmission characteristics as function of the chosen synaptic scenario.

\section{Biophysical model comprising pre- and postsynaptic mechanisms}

Still, a quantitative dynamic model comprising pre- and postsynaptic processes, which is able to capture and explain the diversity of experimental observations, is missing. In this 
work we design a model of synaptic transmission by combining theoretical approaches to model both, presynaptic and postsynaptic processes. Notice, that experiments on central synapses suggest that synaptic transmission is influenced by a variety of factors, such as postsynaptic receptor number, amount of neurotransmitter released, synaptic morphology and the anatomy of the synaptic cleft, and that these properties most probably vary from one synapse to the next (Clements, 1996; Edwards, 1995a; Frerking and Wilson, 1996; Harris and Kater, 1994; Liu et al., 1999; Liu and Tsien, 1995; Min et al., 1998; Oleskevich et al., 1999; Rossi et al., 1995; Silver et al., 1996; Walmsley et al., 1998). Therefore our aim is to derive a model as simple, transparent and general as possible and, yet, detailed enough to comprise the essential underlying physiological mechanisms to account for specific conditions at distinct synaptic connections.

\subsection{Overview and objectives of this work}

The main goal of this work is the design of a biophysically motivated theoretical model of synaptic transmission, which quantifies the propagation of signals from the presynaptic towards the postsynaptic side on the basis of modeling the essential underlying physiological mechanisms. We pursue this aim by successively describing the sequence of processes that evolve from the presynaptic towards the postsynaptic side after the arrival of an AP. Step by step we will derive a model for presynaptic vesicles dynamics (Chapter 2), the dynamics of transmitter in the cleft (Chapter 3) and its interaction with postsynaptic receptors (Chapter 4). These different model-parts will be combined in (Chapter 5) to describe a single synaptic connection between two neurons, such that the postsynaptic response can be computed for a given presynaptic input.

The work is organized as follows:

Motivated by experimental work on a central mammalian synapse in the auditory pathway we will derive a model of presynaptic mechanisms in Chapter 2 that goes beyond the commonly used simple depletion model of vesicle release. In our approach we will consider stochastic release of presynaptic vesicles from two pools, which differ concerning the probability of release and recruitment of vesicles after exocytosis, as well as calcium related facilitation mechanisms. It will be shown that our model is able to account for the experimentally observed features of synaptic short-term depression. This is due to an intrinsic, i.e. calcium-independent, activity induced extra-recruitment of vesicles, which occurs as consequence of the different release-probabilities and rates of recruitment of the two pools. The work of this chapter has been done in cooperation with Erwin Neher and Ralf Schneggenburger who in the Department of Membrane Biophysics at the MPI für Biophysikalische Chemie in Göttingen have initiated and performed the experiments this chapter is based on.

Chapter 3 deals with the dynamics of neurotransmitter in the cleft. We present a simple model of the synaptic cleft and analytically compute the spatio-temporal concentration profile of transmitter after exocytosis from a vesicle. It will be demonstrated that trans- 
mitter motion within the cleft can be considered as a two-dimensional diffusion process. We will provide an estimate of the effective diffusion coefficient, which turns out to be considerably smaller than in aqueous solution.

In Chapter 4 we use the complementary theoretical approaches of Monte Carlo simulations and master equations to calculate miniature EPSCs and to compare our results to experimental recordings from hippocampal and brainstem interneurons. We find that the population of postsynaptic receptors in single bouton-like synapses is almost saturated by the release of single vesicles and that long-term changes in synaptic efficacy are most effectively achieved by an increase in receptor number and drastic structural modifications as suggested by Edwards (1995b). It is demonstrated that the theoretical analysis of distributions of amplitudes, rise and decay times of miniature currents yields information about the specific synaptic properties, in particular the synaptic morphology. The work on brainstem interneurons is directly related to experiments by Stefan Titz and Bernhard Keller in the Zentrum für Physiologie at the Georg-August University of Göttingen.

In Chapter 5 the modeling approaches of Chapters 2 to 14 are combined in a computer model of synaptic transmission. Simulations of the complete process of synaptic transmission are performed to generate individual postsynaptic responses of single synaptic connections: For a given stimulus protocol the change in presynaptic calcium is calculated, vesicles are released (considering a calcium dependent release-machinery) and the activation of postsynaptic receptors due to the exocytosis of neurotransmitter is computed. We find that synapses depress during repeated stimulation due to depletion of presynaptic vesicles as well as due to receptor desensitization. Only for stimulation frequencies below $50 \mathrm{~Hz}$ depression is caused solely presynaptically by depletion of vesicles. Under physiological conditions the steady-state depression current is shown to exhibit a frequency-dependency which allows a rate-coded propagation of the input stimulus over a wide frequency-range. This range of frequencies is limited for elevated release-probabilities and furthermore depends on the specific properties of the presynaptic release-machinery. 


\section{Presynaptic vesicle dynamics}

Synapses in the CNS are generally very small in size and hence do not allow a direct application of the initiating stimulus to a single presynaptic terminal. That is the reason why many models of presynaptic vesicle dynamics are based on studies of experimentally accessible synapses, such as the neuromuscular junction (Bain and Quastel, 1992; Betz, 1970; del Castillo and Katz, 1954; Katz and Miledi, 1968; Liley and North, 1953; Quastel, 1997; Zucker, 1996), neuroendocrine cells (Heinemann et al., 1993), or connections in invertebrates (Gingrich and Byrne, 1985; Kusano and Landau, 1975; Ravin et al., 1999). However, these experimental model systems differ in function and complexity, making a transfer of the derived models to central synaptic mechanisms questionable.

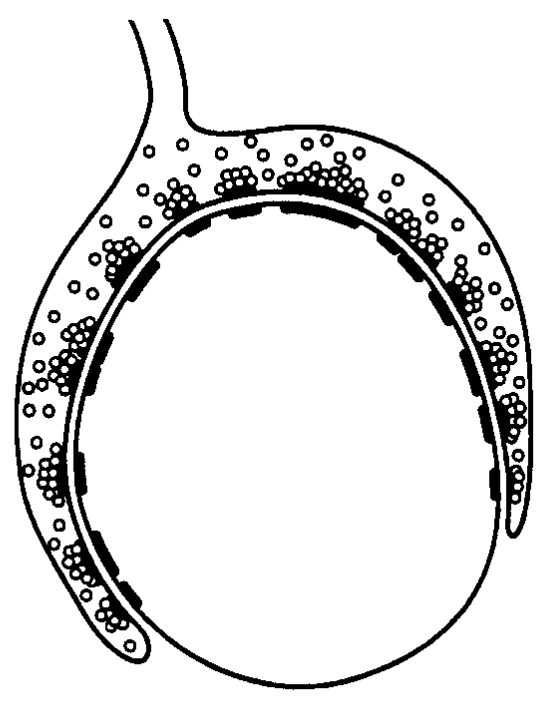

Figure 2.1.: Schematic picture of the calyx of Held synapse. The calyx-like presynaptic terminal encloses the postsynaptic cell and contains hundreds of individual synaptic specializations (from Walmsley et al., 1998).

Recently simultaneous electrophysiological recordings from the pre- and postsynaptic terminal of the calyx of Held, a giant excitatory central synapse in the mammalian auditory pathway (Held, 1893), have become feasible and allow a direct experimental approach to 
presynaptic mechanisms of central synaptic transmission (Borst et al., 1995; Forsythe, 1994).

The calyx of Held synapse (Fig. 2.1) is a fast excitatory, glutamate mediated connection, which is large in size (diameter of $\sim 12 \mu \mathrm{m}$ ) and contains hundreds of individual synaptic specializations. Although quite specific in function and morphology, the calyx of Held can be considered as an ensemble of individual, in parallel operating synaptic connections, activated by the same presynaptic input and acting on the same postsynaptic target. We therefore consider it as a useful model system to study synaptic transmission in the CNS.

During repetitive presynaptic stimulation EPSC amplitudes decay until reaching a steadystate level of depression (von Gersdorff et al., 1997; Weis et al., 1999). This decline of amplitudes occurs to the same extent in AMPA and NMDA mediated EPSCs (von Gersdorff et al., 1997), as well as under block of desensitization (Wang and Kaczmarek, 1998). Therefore it has been suggested that depression is induced presynaptically and reflects the depletion of presynaptic resources (von Gersdorff et al., 1997; Schneggenburger et al., 1999; Weis et al., 1999).

In the following chapter we will evaluate experimental observations during depression to derive a theoretical model of the underlying presynaptic mechanisms. An early approach to model release and recruitment of vesicles, the simple depletion model, dates back to Liley and North (1953). In this model every action potential (AP) depletes the pool of readily-releasable vesicles by a constant fraction, while the pool is simultaneously replenished with a single slow time constant. Several studies of synaptic short-term depression have revealed the limits of this model (Neher, 1998b; Weis et al., 1999; Zucker, 1989). At the calyx of Held the simple depletion model underestimates the observed steady-state activity during depression (Weis et al., 1999). Attempts to extend the simple depletion model by adding an activity dependent extra-recruitment of releasable vesicles (Dittmann et al., 2000; Gingrich and Byrne, 1985; Heinemann et al., 1993; Kusano and Landau, 1975; Stevens and Wesseling, 1998) are not supported by experiments at the calyx of Held (Weis et al., 1999; Wu and Borst, 1999). Nevertheless, experimental attempts to empty the pool of readily-releasable vesicles by strong continuous presynaptic stimulation yield an additional slow current contribution which points to an activity-induced extra-release of vesicles (Wu and Borst, 1999).

This observation has motivated us to modify the simple depletion model by including a second pool of more reluctantly-releasable vesicles in our theoretical model. Following an AP we will consider simultaneous release of presynaptic vesicles from two pools which differ in their probability of release. The major part of vesicles rests in pool $\mathbf{2}$ and is easily released, whereas a smaller fraction of vesicles in pool $\mathbf{1}$ exhibits a much lower releaseprobability. This idea of two types of readily-releasable vesicles which differ regarding the probability of release may serve as a first approximation to a continuum of heterogeneous probabilities of release. It should be noted that experimental evidence for a variability in release-probabilities has been obtained for hippocampal synapses (Rosenmund et al., 1993; Hessler et al., 1993; Murthy et al., 1997).

In our model vesicles in pool $\mathbf{2}$ are only recruited via pool 1, with a much slower time 
constant than the one for refilling of pool 1. We will show that this sequential two-pool model of vesicle release and recruitment contains an intrinsic dynamic of activity dependent vesicle replenishment and manages to explain a variety of experimental findings.

This chapter is organized as follows: We will introduce our model of vesicle dynamics (Sec. 2.1), as well as the implementation of resting conditions and evoked release (Sec. 2.2). Subsequently we will extend the model in order to account for mechanisms of presynaptic facilitation of release, which occur during high-frequency stimulation (Secs. 2.3). Finally we will compare the model to experiments (Sec. 2.5), discuss the results and suggest how to further validate our approach experimentally (Sec. 2.6).

\subsection{Two-Pool-model of vesicle recruitment}

The simple depletion model fails to account for experimental observations at the calyx of Held and a calcium related enhanced recruitment of vesicles failed to be verified experimentally (Weis et al., 1999). Based on the observation of two distinct time-scales during recovery from strong depletion ( $\mathrm{Wu}$ and Borst, 1999) we extend the simple depletion model by adding a second type of reluctantly-releasable vesicles. Like the immediatelyreleasable pool of vesicles we assume them to be located close to the cell membrane within the area of the active zone. Upon arrival of an AP, vesicles from both pools may be released. Reluctantly-releasable vesicles (pool 1, see Fig. 2.2) are released with a release-probability $w_{1}$, which is lower than the release-probability $w_{2}$ for vesicles from the immediately-releasable pool (pool 2).

Ultra-structural studies show that presynaptic vesicles are clustered adjacent to a specialization of the presynaptic membrane, the so called active zone (see for instance Edwards, 1995a). While small bouton-like synapses mostly exhibit a single active zone, which contains approximately four to ten docked vesicles (Schikorski and Stevens, 1997), several hundred active zones have been estimated for calyceal synapses (Lenn and Reese, 1966). It is not well understood how individual presynaptic docking-sites relate to the morphologically defined active zone, e.g. the exact number of functional release-sites per active zone is not known. Electrophysiological recordings at the calyx of Held yield an estimate of about 700 readily-releasable vesicles (Schneggenburger et al., 1999). Furthermore the analysis of fluctuations of EPSCs indicates that maximum amplitudes are caused by approximately $80 \%$ of the available presynaptic resources (Meyer, 1999). We therefore assume that the presynaptic terminal contains a limited number of $n_{\text {tot }}$ release-sites, whereof approximately twenty percent are empty and the rest is either occupied with immediately-releasable or reluctantly-releasable vesicles. In our model the $n_{\text {tot }}$ releasesites are identical, operate independently and appear either empty or occupied by a vesicle from pool 1 or $\mathbf{2}$ (see Fig. 2.2). Empty release-sites are refilled with vesicles of pool 1 with the constant rate $k_{r}$, which implies that recruiting of releasable vesicles occurs activityindependently and is provided by an infinite reserve pool. Experiments studying recovery from depletion ( $\mathrm{Wu}$ and Borst, 1999) allow the conclusion that the total number of re- 
leasable vesicles recovers faster than the immediately-releasable vesicles in pool 2 . In our model a release-site filled with a pool 2 -type vesicle has emerged from being occupied by a vesicle of pool 1 first. Transitions between the states of occupancy occur with the rates as denoted in Fig. 2.2. Vesicles from pool $\mathbf{1}$ are released with the probability $w_{1}$, which is lower than the probability of release $w_{2}$ for a vesicle from pool $\mathbf{2}$. Once a vesicle is released, the release-site immediately returns into the empty state.

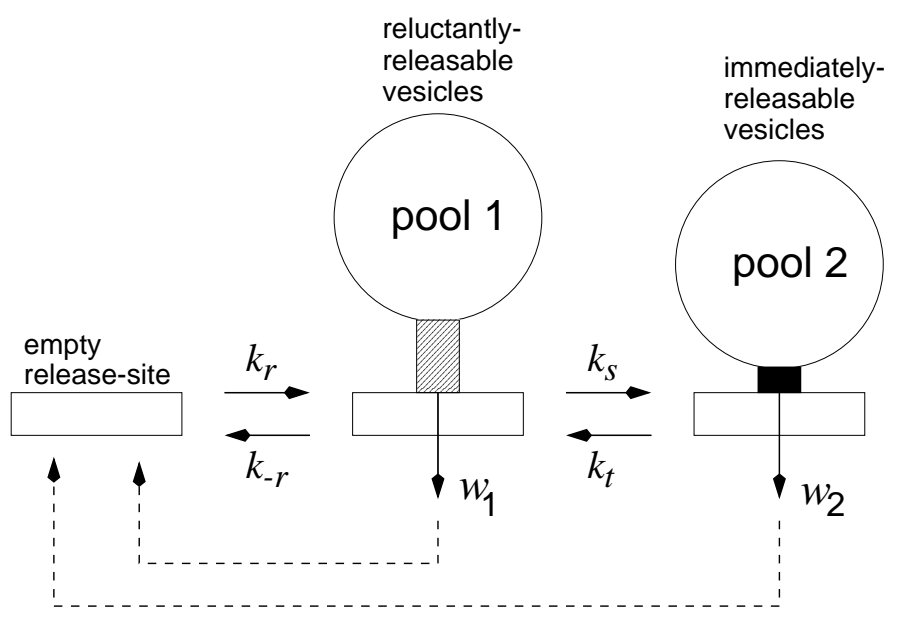

Figure 2.2.: Model of vesicle recruitment and release. Release-sites are either empty, occupied with immediately-releasable vesicles (pool $\mathbf{2}$, release-probability $w_{2}$ ) or filled with more reluctantly-releasable vesicles (pool 1, release-probability $\left.w_{1}\right) ; w_{2}>w_{1}$. Transition rates $k_{r}, k_{-r}, k_{s}$ and $k_{t}$ denote the corresponding kinetic rate constants for filling of sites. Vesicles of pool $\mathbf{2}$ have to be recruited via pool 1. Once a vesicle is released the release-site immediately returns into the empty state.

On the basis of the kinetic scheme in Fig. 2.2 (and neglecting spontaneous release events in the absence of presynaptic stimulation) we are able to formulate master-equations for the occupancy-probabilities $p_{0}(t), p_{1}(t)$ and $p_{2}(t)$ of a single release-site to be empty or filled with a vesicle from pool $\mathbf{1}$ or pool $\mathbf{2}$ :

$$
\begin{aligned}
\frac{d}{d t} p_{0} & =-k_{r} p_{0}+k_{-r} p_{1} \\
\frac{d}{d t} p_{1} & =-\left(k_{s}+k_{-r}\right) p_{1}+k_{r} p_{0}+k_{t} p_{2} \\
\frac{d}{d t} p_{2} & =-k_{t} p_{2}+k_{s} p_{1}
\end{aligned}
$$

with $k_{r}, k_{-r}, k_{s}$ and $k_{t}$ denoting the transition rates between the different states of occupancy as assigned according to Fig. 2.2. As the release-site is either empty or filled with vesicles from pool $\mathbf{1}$ or $\mathbf{2}$, the relation

$$
p_{0}(t)+p_{1}(t)+p_{2}(t)=1
$$


holds.

At rest the ratio of sites occupied with vesicles from pool $\mathbf{1}$ and $\mathbf{2}$ is given by the steadystate solutions of Eqs. 2.1]

$$
p_{1}^{s}=\frac{k_{r} k_{t}}{k_{s} k_{r}+k_{-r} k_{t}+k_{r} k_{t}} \quad \text { and } \quad p_{2}^{s}=\frac{k_{r} k_{s}}{k_{s} k_{r}+k_{-r} k_{t}+k_{r} k_{t}} .
$$

For given initial conditions $p_{1}\left(t_{0}\right)$ and $p_{2}\left(t_{0}\right)$ at time $t_{0}$ the solutions of Eqs. 2.1 yield the time-evolution of the occupancy-probabilities $p_{1}(t)$ and $p_{2}(t)$ :

$$
\begin{aligned}
p_{1}(t)= & \frac{\mathrm{e}^{-\left(t-t_{0}\right) / \tau_{P 1}}}{2 \Omega}\left[\left(k_{r}+k_{-r}+k_{s}-k_{t}+\Omega\right) \Delta p_{1}+2\left(k_{r}-k_{t}\right) \Delta p_{2}\right] \\
& +\frac{\mathrm{e}^{-\left(t-t_{0}\right) / \tau_{P 2}}}{2 \Omega}\left[-\left(k_{r}+k_{-r}+k_{s}-k_{t}-\Omega\right) \Delta p_{1}-2\left(k_{r}-k_{t}\right) \Delta p_{2}\right]+p_{1}^{s}, \\
p_{2}(t)= & \frac{\mathrm{e}^{-\left(t-t_{0}\right) / \tau_{P 1}}}{2 \Omega}\left[-2 k_{s} \Delta p_{1}-\left(k_{r}+k_{-r}+k_{s}-k_{t}-\Omega\right) \Delta p_{2}\right] \\
& +\frac{\mathrm{e}^{-\left(t-t_{0}\right) / \tau_{P 2}}}{2 \Omega}\left[2 k_{s} \Delta p_{1}+\left(k_{r}+k_{-r}+k_{s}-k_{t}+\Omega\right) \Delta p_{2}\right]+p_{2}^{s},
\end{aligned}
$$

where

$$
\begin{aligned}
\Delta p_{j} & =p_{j}\left(t_{0}\right)-p_{j}^{s}, \quad(j=1,2), \\
\tau_{P 1}^{-1} & =\frac{1}{2}\left(k_{r}+k_{-r}+k_{s}+k_{t}+\Omega\right), \\
\tau_{P 2}^{-1} & =\frac{1}{2}\left(k_{r}+k_{-r}+k_{s}+k_{t}-\Omega\right), \\
\Omega & =\sqrt{\left(k_{r}+k_{-r}+k_{s}-k_{t}\right)^{2}+4 k_{s}\left(k_{t}-k_{r}\right)},
\end{aligned}
$$

and $\tau_{P 1}$ and $\tau_{P 2}$ denoting the two intrinsic time-constants. The dynamics is completely determined if the four kinetic rate constants $k_{i},(i=r,-r, s, t)$ are known. Notice that the kinetic rate constants do not directly correspond to measurable quantities (Colquhoun and Hawkes, 1995). Hence we have to express the four kinetic rates $k_{i}$ in terms of four experimentally accessible observables.

Two of the kinetic rate constants are defined by identifying the two intrinsic time-constants $\tau_{P 1}$ and $\tau_{P 2}$ (Eqs. 2.4) with the two clearly distinguishable time-constants in the joint recovery of both pools after complete depletion (Wu and Borst, 1999). The remaining two kinetic rate constants are first expressed by means of the occupancy of release-sites at rest, which reaches about $80 \%$ (Meyer, 1999). We define the ratio $F$ of filled release-sites at rest, which is given as fraction of the joint number of vesicles in pool $\mathbf{1}$ and pool $\mathbf{2}$, $n_{\text {rel }}=n_{1}+n_{2}$, divided by the total number of release-sites, $n_{\text {tot }}$,

$$
F:=\frac{n_{\text {rel }}}{n_{\text {tot }}}=p_{1}^{s}+p_{2}^{s} .
$$


Second, we consider the ratio at steady-state between the two pools (Neher, 1999). We define this quantity using the parameter $R(0<R<1)$, such that the ratio between the two pools in the steady-state is given by

$$
p_{1}^{s}: p_{2}^{s}=R:(1-R) \text {. }
$$

This yields the kinetic rate constants $k_{r}, k_{-r}, k_{s}$ and $k_{t}$ in terms of $F, R$ and the two time-constants $\tau_{P 1}$ and $\tau_{P 2}:$ !

$$
\begin{aligned}
k_{-r} & =\frac{1-F}{2(1-F+F R)}\left[\Lambda_{+}( \pm) \sqrt{\Lambda_{+}^{2}+\frac{1}{R}(1-F+F R)\left(\Lambda_{-}^{2}-\Lambda_{+}^{2}\right)}\right], \\
k_{s} & =(R-1)\left[k_{-r} \frac{1+F(R-1)}{1-F}-\Lambda_{+}\right] \\
k_{r} & =k_{-r} \frac{F R}{1-F} \quad \text { and } \quad k_{t}=k_{s} \frac{R}{1-R},
\end{aligned}
$$

where $\Lambda_{+}=1 / \tau_{P 1}+1 / \tau_{P 2}$ and $\Lambda_{-}=1 / \tau_{P 1}-1 / \tau_{P 2}$.

\subsection{Resting conditions and release following a single action potential}

At rest the system is in steady-state, i.e. the number of release-sites occupied with vesicles from pool 1 or $\mathbf{2}$ is given by the corresponding steady-state values $n_{\text {tot }} p_{1}^{s}$ and $n_{\text {tot }} p_{2}^{s}$ in Eq. 2.2. An AP at time $t_{\mathrm{AP}}$ causes release of vesicles from both pools: the number of vesicles released is proportional to the respective pool-occupancy and the corresponding release-probability. If we assume that the postsynaptic current is directly proportional to the number of vesicles released the AP-generated EPSC is given by

$$
I_{\mathrm{AP}}\left(t_{\mathrm{AP}}\right)=Q n_{\mathrm{tot}}\left[w_{1} p_{1}^{+}\left(t_{\mathrm{AP}}, t_{0}\right)+w_{2} p_{2}^{+}\left(t_{\mathrm{AP}}, t_{0}\right)\right],
$$

with $Q$ denoting a proportionality factor assigned to the release of a single vesicle. Here $p_{j}^{+}\left(t_{\mathrm{AP}}, t_{0}\right) \quad(j=1,2)$ denotes the pool-occupancy immediately before the release of vesicles at time $t=t_{\mathrm{AP}}$ for given initial conditions at time $t=t_{0}$. Right after the release of vesicles the pool-occupancy is given by $p_{j}^{-}\left(t_{\mathrm{AP}}\right)(j=1,2)$, which is

$$
p_{1}^{-}\left(t_{\mathrm{AP}}\right)=\left(1-w_{1}\right) p_{1}^{+}\left(t_{\mathrm{AP}}, t_{0}\right) \quad \text { and } \quad p_{2}^{-}\left(t_{\mathrm{AP}}\right)=\left(1-w_{2}\right) p_{2}^{+}\left(t_{\mathrm{AP}}, t_{0}\right) .
$$

If no further stimulation occurs, both pools recover towards their resting states according to Eqs. 2.3, initial conditions in these equations given by $p_{1}^{-}\left(t_{0}\right)$ and $p_{2}^{-}\left(t_{0}\right)$, respectively.

\footnotetext{
${ }^{1}$ The two solutions for $k_{-r}$ in Eqs. 2.7 represent the two possibilities to assign the observed timeconstants $\tau_{P 1}$ and $\tau_{P 2}$ to the two transitions between the three occupancy-states. The recovery from complete depletion indicates that pool 1 is refilled with a very fast time-constant of $\tau_{P 1} \sim 0.3 \mathrm{~s}$, while the recovery of pool $\mathbf{2}$ occurs on a much slower time-scale ( $\mathrm{Wu}$ and Borst, 1999). Hence we identify the fast time-constant with the refilling of pool 1, which corresponds to the "+"-solution of $k_{-r}$.
} 


\subsection{Facilitation of release-probability}

Although the overall pattern during stimulus trains is a decay of EPSC amplitudes, the opposite effect, i.e. the facilitation of subsequent amplitudes, has also been observed under certain conditions (Schneggenburger et al., 1999). While the depression is widely attributed to depletion of the pool of readily-releasable vesicles, the detailed mechanisms of facilitation are not clear at all.

A common idea, the so called residual calcium hypothesis (Katz and Miledi, 1968), correlates the increase in EPSC amplitudes during repetitive stimulation with the simultaneously observed rise in the global presynaptic calcium concentration. The global calcium accumulates during repetitive activity in the presynaptic terminal and is thought to enhance the probability of release, whereas the detailed mechanisms causing the effects of global residual calcium on the release-process remain unclear (Fisher et al., 1997; Zucker, 1999).

In the following we will present a model of presynaptic facilitation based on ideas about buffered diffusion of $\mathrm{Ca}^{2+}$ in the presynaptic terminal (see Neher, 1998a for a review). This approach will allow us to compute alterations in the two release-probabilities $w_{1}$ and $w_{2}$ due to changes in residual as well as extracellular calcium concentrations. The detailed physiological mechanisms of facilitation are point of an ongoing debate and we will additionally show that the facilitation of release probability as derived and subsequently used within our model does not crucially depend on assumptions about the underlying mechanisms: An alternative approach attributes calcium related facilitation of release to a high affinity $\mathrm{Ca}^{2+}$-binding site responding to global $\mathrm{Ca}^{2+}$-changes (Bertram et al., 1996; Dittmann et al., 2000; Yamada and Zucker, 1992). We will compare the two facilitationmodels and present a coherent picture of the release-probability as function of changes in global calcium within the two approaches.

Here we are primarily interested in a model of facilitated release due to repeated stimulation, which yields a quantitative description of changes in release-probability with alterations in intra- and extra-cellular calcium. We address this problem on the basis of previous theoretical work on buffered calcium diffusion within the presynaptic terminal (Naraghi and Neher, 1997; Neher, 1986; Neher, 1998a). Within this approach the local calcium which initiates the release of vesicles is influenced by the global, spatially averaged calcium concentration and hence varies with changes in the global calcium concentration. The details of our approach are explained in Appendix $\mathrm{A}$ and will be summarized in the following.

\subsubsection{Buffered calcium diffusion and facilitation of release}

To include facilitation we first take into account that four calcium binding sites are required to activate transmitter release (Borst and Sakmann, 1996; Dodge and Rahamimoff, 1967; Heidelberger et al., 1994; Schneggenburger et al., 1999). We use a Hill equation with 
a forth-order dependency of the release-probability $w_{\text {rel }}$ on the local $\mathrm{Ca}^{2+}$-concentration $\left[\mathrm{Ca}^{2+}\right]_{\mathrm{RS}}$ at a presynaptic release-site,

$$
w_{\mathrm{rel}}=w_{\max } \frac{\left[\mathrm{Ca}^{2+}\right]_{\mathrm{RS}}^{4}}{\left[\mathrm{Ca}^{2+}\right]_{\mathrm{RS}}^{4}+K_{1 / 2}^{4}},
$$

with $K_{1 / 2}$ denoting the calcium concentration for half maximal release and $w_{\max }$ indicating maximal release.

Local domains of high calcium concentration exist in the close vicinity of open $\mathrm{Ca}^{2+}$ channels or clusters of $\mathrm{Ca}^{2+}$-channels and are thought to play a major role in controlling the mechanisms of neurotransmitter release (Aharon et al., 1994; Chad and Eckert, 1984; Fogelson and Zucker, 1985; Neher, 1998b; Rios and Stern, 1997; Simon and Llinas, 1985). When calcium enters the presynaptic terminal through open channels local $\mathrm{Ca}^{2+}$-microdomains build up quickly and are strongly influenced by the presence of mobile $\mathrm{Ca}^{2+}$-buffers (Naraghi and Neher, 1997; Neher, 1998a; Roberts, 1994). Theoretical studies point towards microdomains exhibiting $\mathrm{Ca}^{2+}$-concentrations as high as $100 \mu \mathrm{M}$ (Yamada and Zucker, 1992), whereas experimental estimates of these local concentrations are missing. In contrast estimates of the global $\mathrm{Ca}^{2+}$-concentration are known from experiments with $\mathrm{Ca}^{2+}$-indicator dyes (Takahashi et al., 1999).

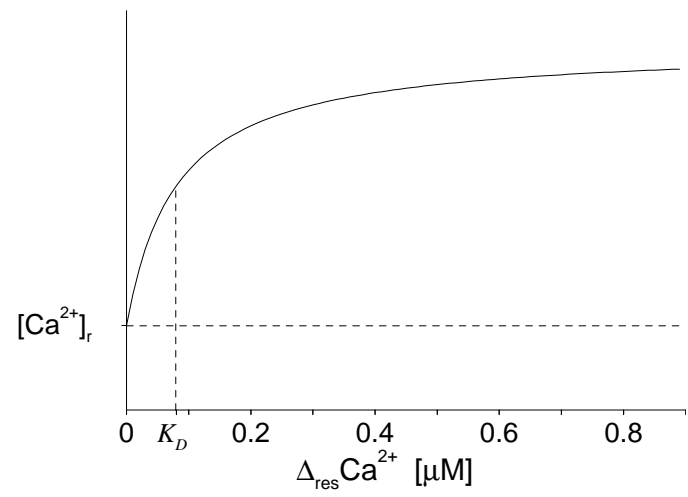

Figure 2.3.: Dependence of the local calcium concentration $\left[\mathrm{Ca}^{2+}\right]_{\mathrm{RS}}$ on changes in residual calcium $\Delta_{\text {res }} \mathrm{Ca}^{2+}$ as computed from Eq. 2.10. The parameter $K_{D}$ denotes the elevation in $\Delta_{\text {res }} \mathrm{Ca}^{2+}$ for half-maximal increase in $\left[\mathrm{Ca}^{2+}\right]_{\mathrm{RS}}$.

We assume that the local calcium concentration at the release-site $\left[\mathrm{Ca}^{2+}\right]_{\mathrm{RS}}$ increases with changes in the global residual calcium $\Delta \mathrm{Ca}_{\text {res }}^{2+}$ and also depends on the calcium influx $J_{\mathrm{Ca}}\left(\left[\mathrm{Ca}^{2+}\right]_{\text {out }}\right)$, which is a function of the extracellular calcium concentration $\left[\mathrm{Ca}^{2+}\right]_{\text {out }}$. The dependence of $\left[\mathrm{Ca}^{2+}\right]_{\mathrm{RS}}$ on the residual calcium concentration $\Delta \mathrm{Ca}_{\text {res }}^{2+}$ is modeled (for $\left.\Delta \mathrm{Ca}_{\text {res }}^{2+}>0\right)$ by

$$
\left[\mathrm{Ca}^{2+}\right]_{\mathrm{RS}}=\left[\mathrm{Ca}^{2+}\right]_{\mathrm{r}}+J_{\mathrm{Ca}}\left(\left[\mathrm{Ca}^{2+}\right]_{\mathrm{out}}\right) \alpha\left[1+\eta\left(1-\frac{\gamma}{1+\frac{\Delta_{\mathrm{res}} \mathrm{Ca}^{2+}}{K_{D}}}\right)\right] .
$$


with $\alpha, \eta$, and $\gamma$ denoting free model parameters and $K_{D}$ the effect of half-maximal elevation of $\left[\mathrm{Ca}^{2+}\right]_{\mathrm{RS}}$. The elevation of $\left[\mathrm{Ca}^{2+}\right]_{\mathrm{RS}}$ above resting level due to changes in residual calcium is illustrated in Fig. 2.3]. In Appendix A it is explained in detail how Eq. 2.10 can be derived under the assumption that the presynaptic calcium interacts with mobile calcium buffers. After entering the presynaptic terminal the calcium rapidly equilibrates with two endogenous calcium buffers, one being present in high, the second in low concentration and almost saturated by the global calcium (see Appendix A). The influx of calcium $J_{\mathrm{Ca}}$ is taken to be constant for a given extracellular calcium concentration $\left[\mathrm{Ca}^{2+}\right]_{\text {out }}$.

Variations in extracellular $\mathrm{Ca}^{2+}$ are often used to experimentally manipulate the probability of release. We include effects of altered extra cellular $\mathrm{Ca}^{2+}$-concentration by taking into account that $\mathrm{Ca}^{2+}$-influx saturates with increasing concentrations of extra-cellular calcium $\left[\mathrm{Ca}^{2+}\right]_{\text {out }}$ (Church and Stanley, 1996; Schneggenburger et al., 1999). Measurements indicate that $\mathrm{Ca}^{2+}$-influx exhibits strong saturation (half-maximal $\mathrm{Ca}^{2+}$-influx at $\left.\mathrm{EC}_{50} \sim 2.6 \mathrm{mM}\left[\mathrm{Ca}^{2+}\right]_{\text {out }}\right)$ and is described by a Michaelis-Menten saturation equation (Schneggenburger et al., 1999)

$$
J_{\mathrm{Ca}}\left(\left[\mathrm{Ca}^{2+}\right]_{\text {out }}\right)=J_{\mathrm{Ca}, \max } \frac{\left[\mathrm{Ca}^{2+}\right]_{\text {out }}}{\left[\mathrm{Ca}^{2+}\right]_{\text {out }}+\mathrm{EC}_{50}},
$$

$J_{\mathrm{Ca}, \max }$ indicating the maximal value of saturation.

Combining Eqs. 2.10 and 2.11 yields the dependency of $\left[\mathrm{Ca}^{2+}\right]_{\mathrm{RS}}$ on the extra-cellular $\mathrm{Ca}^{2+}$-concentration $\left[\mathrm{Ca}^{2+}\right]_{\text {out }}$, as well as on changes of the global residual calcium $\Delta_{\mathrm{res}} \mathrm{Ca}^{2+}$

$$
\left[\mathrm{Ca}^{2+}\right]_{\mathrm{RS}}=\left[\mathrm{Ca}^{2+}\right]_{\mathrm{r}}+J_{\mathrm{Ca}, \mathrm{max}} \frac{\left[\mathrm{Ca}^{2+}\right]_{\mathrm{out}}}{\left[\mathrm{Ca}^{2+}\right]_{\mathrm{out}}+\mathrm{EC}_{50}} \alpha \underbrace{\left[1+\eta\left(1-\frac{\gamma}{1+\frac{\Delta_{\mathrm{res}} \mathrm{Ca}_{D}^{2+}}{K_{D}}}\right)\right]}_{=: \xi\left(\Delta_{\mathrm{res}} \mathrm{Ca}^{2+}\right)} .
$$

Under resting conditions $\Delta_{\mathrm{res}} \mathrm{Ca}^{2+}$ is zero and $\left[\mathrm{Ca}^{2+}\right]_{\mathrm{RS}}$ equals the global basal $\mathrm{Ca}^{2+}{ }_{-}$ concentration $\left[\mathrm{Ca}^{2+}\right]_{\mathrm{r}}$. The constants $\eta, \gamma$ and $\alpha$ (in units of $K_{1 / 2}$ in Eq. 2.9) serve as free fit parameters in our model.

\section{Distinguishing between two vesicle pools}

In the following we will show how to incorporate the facilitation model into our approach of two different vesicle-pools. As suggested for neuroendocrine cells (Klingauf and Neher, 1997; Xu et al., 1999) we follow the idea that a presynaptic release-site of an immediatelyreleasable vesicle $(=$ pool $\mathbf{2})$ is colocalized with a $\mathrm{Ca}^{2+}$-channel. This specific channel might be missing for reluctantly-releasable vesicles from pool 1. It has been suggested that calcium channels of different efficiency can cause release, whereas low efficient channels seem to be located distantly from release-sites (Wu et al., 1999). We will assume that vesicles from pool 1 are exposed to a lower $\mathrm{Ca}^{2+}$-concentration and according to Eq. 2.9 are released with a lower probability. We account for the reduced local $\mathrm{Ca}^{2+}$-concentration 
due to the lack (or inefficiency) of a colocalized $\mathrm{Ca}^{2+}$-channel by setting the first addend in Eq. 2.10 to zero for vesicles of pool 1 (see Appendix A for details).

The release-probability of vesicles in pool $j$ depends on changes in the global residual $\mathrm{Ca}^{2+}$ and the extra-cellular $\mathrm{Ca}^{2+}$-concentration in the following way:

$$
\begin{aligned}
& w_{j}=\frac{\left[\mathrm{Ca}^{2+}\right]_{\mathrm{RS}}^{4}}{\left[\mathrm{Ca}^{2+}\right]_{\mathrm{RS}}^{4}+K_{1 / 2}^{4}}, \quad j=1,2 \\
& \text { with }
\end{aligned}
$$

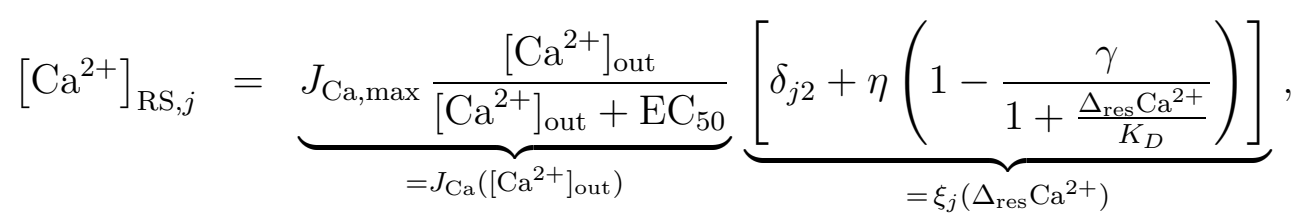

with $\delta_{j 2}(j=1,2)$ denoting Kronecker's symbol. Here $K_{1 / 2}, \eta, \gamma$ and $K_{D}$ are free parameters of the model. The values for $J_{\mathrm{Ca} \text {,max }}$ and $\mathrm{EC}_{50}$ have been estimated by measurements with varying concentrations of $\left[\mathrm{Ca}^{2+}\right]_{\text {out }}$ (Schneggenburger et al., 1999). Notice, that two of the above four model parameters are determined by the choice of the release-probabilities at rest, i.e. $w_{j}\left(\Delta_{\text {res }} \mathrm{Ca}^{2+}=0\right)$.

\subsubsection{Calcium-binding site model of facilitation}

Even if there seems to be agreement about facilitation being related to changes in the global intracellular calcium, it is not clear, how the residual calcium interacts with the release-machinery. In our approach it is assumed that due to the lack of a colocalized calcium channel, vesicles from pool $\mathbf{1}$ are exposed to a lower local calcium concentration. A different approach has been proposed by Bertram et al. (1996), Dittmann et al. (2000), and Yamada and Zucker (1992). In their model release is triggered by a high-affinity calcium-binding site and inhomogeneities in release-probability are due to different calcium-affinities of the binding site. We subsequently demonstrate that this approach yields alterations in the release-probability with changes in global presynaptic calcium in accordance with the model presented in the previous section.

In the work of Yamada and Zucker (1992), Bertram et al. (1996) and Dittmann et al. (2000) it is assumed that the release of vesicles is controlled by a high affinity $\mathrm{Ca}^{2+}$ binding site, responding to global changes in residual calcium $\Delta_{\text {res }} \mathrm{Ca}^{2+}$, such that the release-probabilities $w_{j}$ vary according to

$$
w_{j}=w_{j}^{0}+\frac{\left[w_{j}^{f f}-w_{j}^{0}\right] \Delta_{\mathrm{res}} \mathrm{Ca}^{2+}}{\Delta_{\mathrm{res}} \mathrm{Ca}^{2+}+K_{d, j}}, \quad j=1,2,
$$

with $K_{d, j}$ denoting the two dissociation constants of the binding sites assigned to pools $\mathbf{1}$ and $2, w_{j}^{0}$ the probability of release at rest, i.e. $\Delta_{\text {res }} \mathrm{Ca}^{2+}=0$, and $w_{j}^{f f}$ at full facilitation $\left(\Delta_{\text {res }} \mathrm{Ca}^{2+}=\infty\right)$. These parameters are treated as free model parameters. 


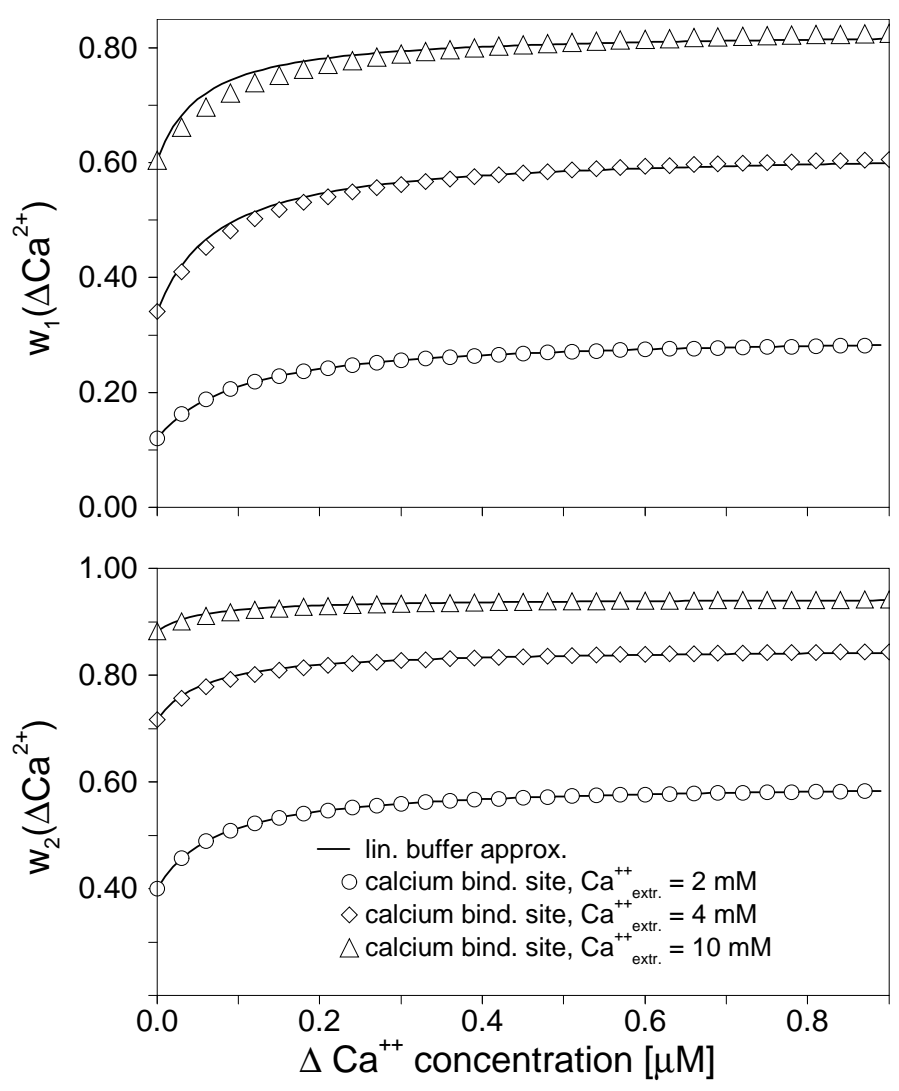

Figure 2.4.: Comparison of the two facilitation models for varying concentrations of $\left[\mathrm{Ca}^{2+}\right]_{\text {out }}$. The solid line corresponds to the buffered diffusion model (Eq. 2.13); see Tab. 2.1 (set 2) for the values assigned to the model parameters. White symbols represent the calcium binding-site model (Eq. 2.14); the values for $K_{d, 1}=0.0985 \mu \mathrm{M}$ and $K_{d, 2}=0.074 \mu \mathrm{M}$ are gained from fitting for $\left[\mathrm{Ca}^{2+}\right]_{\text {out }}=2 \mathrm{mM}$. The rest of parameters $\left(w_{j}^{0}\right.$ and $w_{j}^{f f}$ for $\left.j=1,2\right)$ is chosen to assure equivalent initial release-probabilities and an optimum correspondence between the two models. Top panel: Release-probability $w_{1}$ from pool $\mathbf{1}$ as function of changes in global calcium. Bottom panel: Release-probability $w_{2}$. 
We want to emphasize two points: First, although based on different physiological mechanisms the two approaches reveal very similar quantitative features. As displayed in Fig. 2.4 for a reasonable set of parameters (see Sec. 2.5 and Fig. 2.6 for details), the two facilitation models discussed here exhibit almost the same dependence of both release-probabilities on $\Delta_{\text {res }} \mathrm{Ca}^{2+}$ and coincide within the range of experimental accuracy. Second, due to the lack of knowledge about the physiological mechanisms involved we are not interested in a detailed analysis of the underlying mechanisms, but in formulating a model with a minimal number of parameters. The basic assumption here only concerns the residual calcium hypothesis (Katz and Miledi, 1968) assuming that facilitation is related to an increase in global residual $\mathrm{Ca}^{2+}$, which itself changes as result of presynaptic stimulation.

Although the calcium binding-site approach seems to be based on less specific assumptions, it carries a larger amount of free parameters: In contrast to the facilitation model based on buffered calcium diffusion it is not possible to differentiate between facilitation effects due to variations of the residual calcium or the extra-cellular $\mathrm{Ca}^{2+}$-concentration. An elevated extra-cellular $\mathrm{Ca}^{2+}$-concentration for instance requires a new set of estimates for $w_{j}^{0}$ and $w_{j}^{f f}$, whereas one set of fit parameters in Eqs. 2.13 covers the whole range of $\mathrm{Ca}^{2+}$-dynamics. Hence, for the following calculations we will employ Eqs. 2.13 to model presynaptic facilitation.

\subsection{Calculating EPSCs evoked by a sequence of action potentials}

We calculate the dynamics of the presynaptic spatially averaged $\mathrm{Ca}^{2+}$ - concentration $\left[\mathrm{Ca}^{2+}\right]_{\text {global }}$ using the single compartment model (Neher and Augustine, 1992). In this model the spatially averaged time course of the free $\mathrm{Ca}^{2+}$-concentration is calculated by treating the presynaptic terminal as a single cell compartment. Furthermore it is assumed that the intracellular $\mathrm{Ca}^{2+}$-concentration rapidly equilibrates with calcium buffers. The $\mathrm{Ca}^{2+}$-influx due to an AP is then modeled by an instantaneous increase $x_{0}$ of the global $\mathrm{Ca}^{2+}$-concentration. The residual $\mathrm{Ca}^{2+}$ is brought back to resting level by $\mathrm{Ca}^{2+}$-pumps on a much slower time scale, which is characterized by the time constant $\tau_{x}$. The time course of the free global calcium $\left[\mathrm{Ca}^{2+}\right]_{\text {global }}$ is then given by

$$
\left[\mathrm{Ca}^{2+}\right]_{\text {global }}(t)=\underbrace{x_{0} \mathrm{e}^{-t / \tau_{x}}}_{=\Delta_{\mathrm{res}} \mathrm{Ca}^{2+}(t)}+\left[\mathrm{Ca}^{2+}\right]_{\mathrm{r}} .
$$

The range of $\tau_{x}$ and $x_{0}$ has been measured experimentally for the calyx of Held (Helmchen et al., 1997) and our estimates are displayed in Tab. 2.1.

For repetitive presynaptic stimulation we assume linear superposition of calcium transients due to individual APs as given by Eq. 2.15 (Borst and Sakmann, 1999; Helmchen et al., 1997; Weis et al., 1999). The increase in global residual calcium $\Delta_{n} \mathrm{Ca}^{2+}$ after a sequence of $n$ stimuli, applied with frequency $f_{\text {stim }}$, i.e. corresponding to an inter-stimulus interval 
of $\Delta t=1 / f_{\text {stim }}$ is then given by (if the first stimulus occurs at time $t=0$ ) (Helmchen et al., 1996)

$$
\Delta_{\text {res }}^{(n)} \mathrm{Ca}^{2+}(t)=x_{0} \sum_{v=1}^{n} \mathrm{e}^{-(t-(v-1) \Delta t) / \tau_{x}}, \quad t>(n-1) \Delta t .
$$

An example is displayed in Fig. 2.7C , where the change in global calcium as calculated by Eq. 2.16 is shown for stimulus trains of 10 and $100 \mathrm{~Hz}$.

By summing over Eq. 2.15 we are able to calculate the actual global residual calcium $\Delta \mathrm{Ca}^{2+}(t)$ reflecting and "remembering" the sequence of previous APs. Inserting $\Delta \mathrm{Ca}^{2+}(t)$ into Eqs. 2.13 yields the corresponding, due to previous stimulation possibly facilitated, release-probabilities $w_{1}$ and $w_{2}$. Figures $2.7 D$ and $E$ display an illustration of presynaptic facilitation due to $10 \mathrm{~Hz}$ and $100 \mathrm{~Hz}$ stimulus-trains as calculated by Eqs. 2.13.

During a sequence of several APs the postsynaptic current initiated by the $n$th stimulus is calculated in analogy to Eq. 2.8 by

$$
I_{\mathrm{AP}}^{(n)}\left(t_{n}\right)=Q n_{\text {tot }}\left[w_{1}\left(\Delta_{\text {res }}^{(n-1)} \mathrm{Ca}^{2+}\right) p_{1}^{+}\left(t_{n}, t_{n-1}\right)+w_{2}\left(\Delta_{\text {res }}^{(n-1)} \mathrm{Ca}^{2+}\right) p_{2}^{+}\left(t_{n}, t_{n-1}\right)\right],
$$

where $\Delta^{(n-1)} \mathrm{Ca}^{2+}$ denotes the change in global calcium due to the previous $(n-1)$ stimuli and is computed by Eq. 2.16. The occupancy in pool 1 immediately before release caused by the $n$th stimulus, $p_{1}^{+}\left(t_{n}, t_{n-1}\right)$ is calculated from Eq. 2.3 with initial conditions given by the pool-occupancy $p_{1}^{-}\left(t_{n-1}, t_{n-2}\right)$ immediately after the prior, $(n-1)$ th stimulus

$$
p_{1}^{-}\left(t_{n-1}, t_{n-2}\right)=\left[1-w_{1}\left(\Delta_{\text {res }}^{(n-2)} \mathrm{Ca}^{2+}\right)\right] p_{1}^{+}\left(t_{n-1}, t_{n-2}\right) .
$$

Hence, successively applying Eqs. 2.18 and 2.17 enables us to calculate the postsynaptic current for a given sequence of APs.

We set the initial conditions to the resting conditions in Eq. 2.2 and keep track of effects from subsequent stimuli by "updating" the pool-occupancies $p_{j}$ (using Eqs. 2.18 and 2.3) and release-probabilities $w_{j}$ (Eqs. 2.13 and 2.16). 


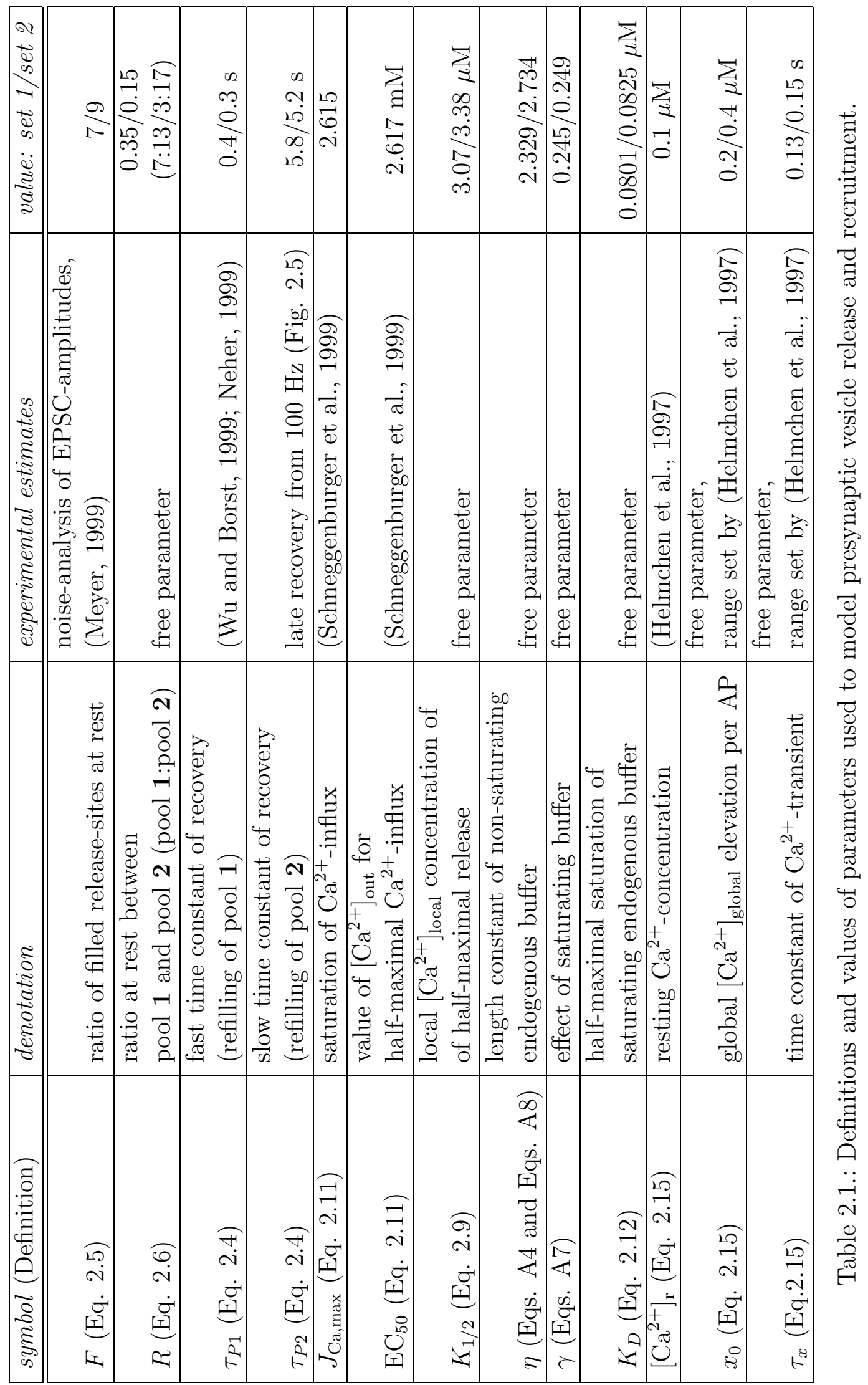




\subsection{Results}

In the previous sections the complete dynamics of release and recruitment of presynaptic vesicles, including facilitation of release due to repetitive stimulation has been formulated. Fitting the model to experiments requires setting the model parameters, which are summarized in Tab. 2.1. Some of these parameters can be identified directly with experimental observables. These are the two time constants of vesicle recruitment, $\tau_{P 1}$ and $\tau_{P 2}$ (Eqs. 2.4), which are known from studying the recovery of synaptic transmission after repetitive high-frequency stimulation (Schneggenburger, 1999) or application of a strong, depolarizing voltage-step pulse (Wu and Borst, 1999). Furthermore the analysis of EPSC-amplitude fluctuations indicates that the release-sites are occupied up to $\sim 80 \%$ (Meyer, 1999), which we use as an estimate for the ratio of filled sites at rest, $R$ (Eq. 2.6).

Data concerning the $\mathrm{Ca}^{2+}$-influx, $J_{\mathrm{Ca} \text {,max }}$ and $\mathrm{EC}_{50}$ (Eq. 2.11), have been measured by Schneggenburger et al. (1999). Although employed as fit parameters, the range of values for $x_{0}$ and $\tau_{x}$ (Eq. 2.15) to model the global $\mathrm{Ca}^{2+}$-dynamics is restricted and chosen close to the estimates given in by Helmchen et al. (1997).

The remaining model-parameters (see Tab. 2.1) are taken as free parameters to fit the model to three sets of experimental data. The first set is given by recordings of the depression of EPSC-amplitudes following a $10 \mathrm{~Hz}$-train of 30 stimuli (von Gersdorff et al., 1997; Weis et al., 1999) (normalized by dividing by the first EPSC response). After approximately 10 stimuli the normalized current reaches a steady-state value (see Fig. 2.5B), which decreases for increasing frequency (von Gersdorff et al., 1997; Weis et al., 1999). Its frequency-dependence is displayed in Fig. 2.5D. The recovery of release from highfrequency stimulation as studied by a test-AP after a time interval $\Delta t$ following the last depressing stimulus (Fig. 2.5A) provides a third set of experimental estimates (Schneggenburger, 1999).

As shown in Figs. 2.5 and 2.6 and given by the values in Tab. 2.1 two sets of parameters have been found, which are able to comprise the experimentally observed depression of EPSC amplitudes, recovery from depression as well as the frequency-dependence of the steady-state depression current. Systematic deviations of theory and experiments are only seen for the response of the second stimulus in a $10 \mathrm{~Hz}$ stimulus train, where the theory fails to reproduce the strong decay in the amplitude of the second stimulus compared to initial amplitude.

It should be taken into account that the experimental data itself exhibit a large variability between individual cells: During a 10-Hz-stimulus train, steady-state level and relative amplitude of response to the second stimulus seem to correlate with absolute amplitude of the first response (von Gersdorff et al., 1997). Larger responses (white rectangles in Fig. 2.6) come along with a stronger relative decay in the second amplitude and reach a lower level of steady-state depression. This effect most probably reflects the abundant use of limited resources by a first strong release. Cross-correlations between subsequent stimuli are currently being studied by an extended theoretical and experimental analysis (Neher, 1999). In this context we want to point out that variations in the kinetic parameters of 

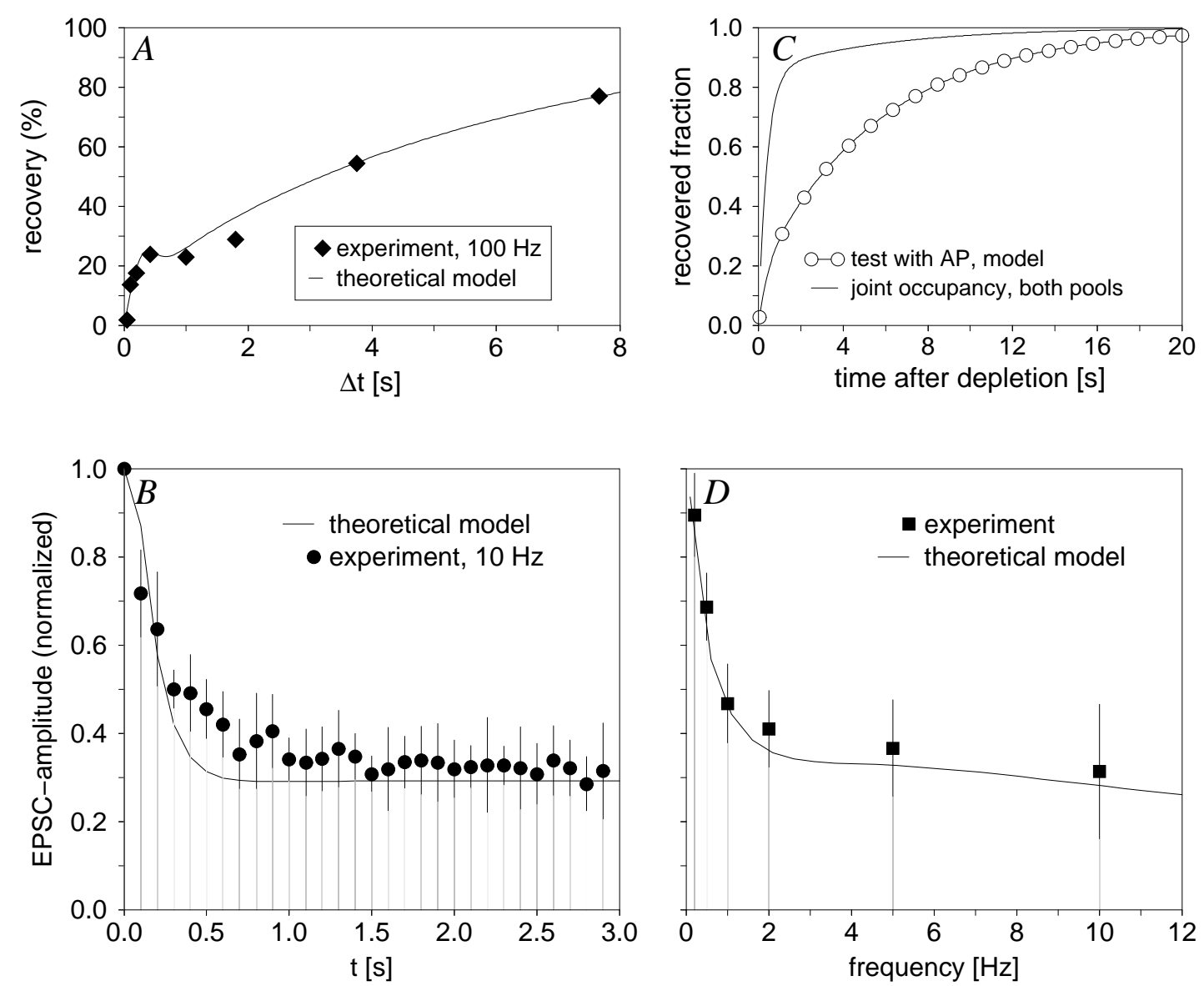

Figure 2.5.: Synaptic depression and recovery from depression as recorded in experiments (black symbols) and calculated by the theoretical model (solid line, parameters from set 1 in Tab. 2.1). A: Recovery as tested by a subsequent AP at $t=\Delta t$ after high-frequency stimulation with $100 \mathrm{~Hz}$. B: Normalized EPSC amplitude during stimulation with $10 \mathrm{~Hz} . C$ : Theoretically calculated recovery from complete depletion, for the joint occupancy in both pools (solid line) and as tested by a subsequent $\operatorname{AP} t=\Delta t$ after complete depletion (white circles). $D$ : Frequency-dependence of the normalized steady-state depression current on the stimulus frequency. 

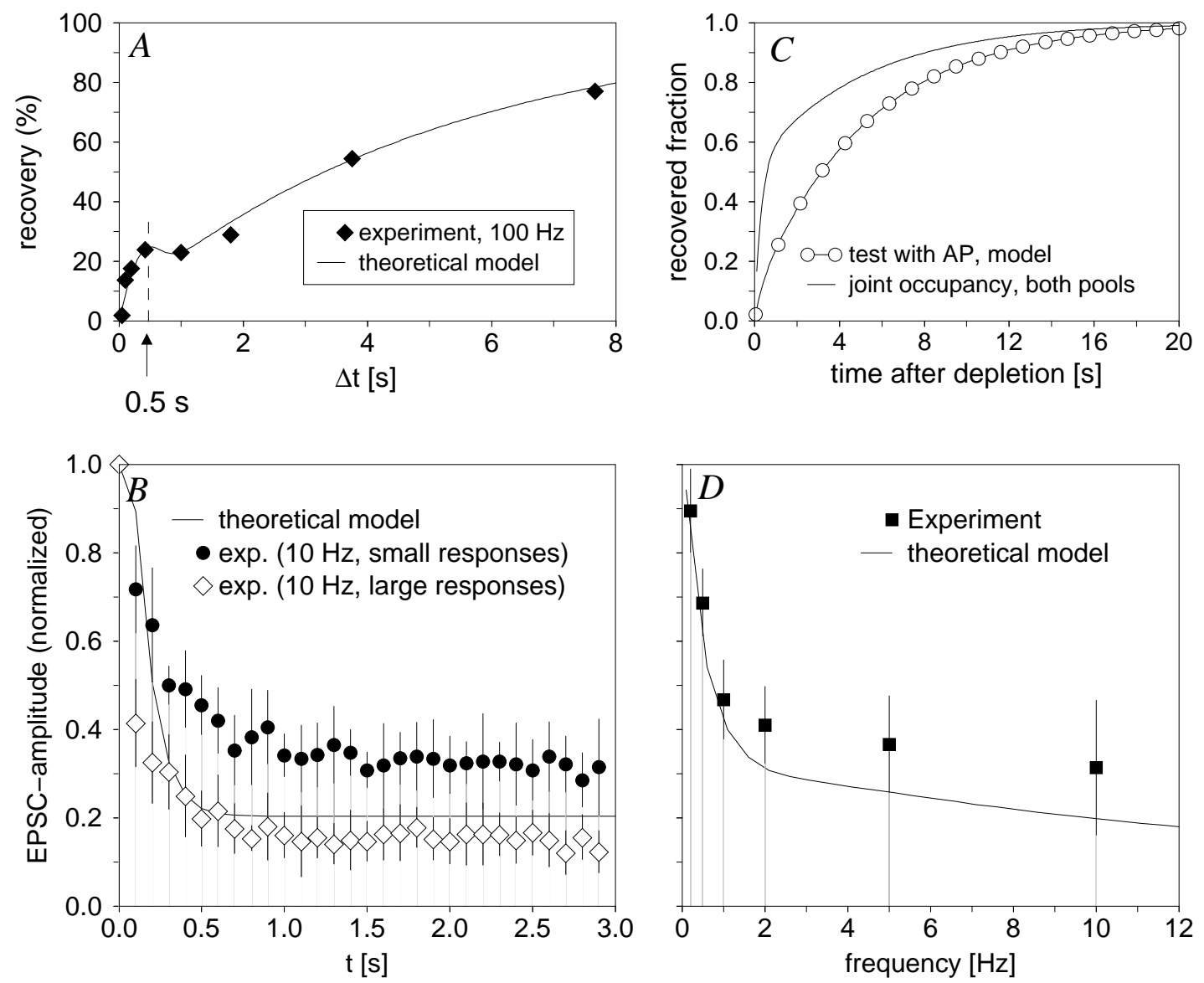

Figure 2.6.: Synaptic depression and recovery from depression as recorded in experiments (black and white symbols) and calculated by the theoretical model (solid line, parameters from set 2 in Tab. 2.1). A: Recovery as tested by a subsequent AP at $t=\Delta t$ after high-frequency stimulation with $100 \mathrm{~Hz}$. $B$ : Normalized EPSC amplitude during stimulation with $10 \mathrm{~Hz}$. Cells with a first large response exhibit a lower steady-state depression current level (white rectangles) than those with a smaller first response (black circles). $C$ : Theoretically calculated recovery from complete depletion, for the joint occupancy in both pools (solid line) and as tested by a subsequent $\mathrm{AP} t=\Delta t$ after complete depletion (white circles). $D$ : Frequency-dependence of the normalized steady-state depression current on the stimulus frequency. 
our model (Tab. 2.1, set 2) also manage to cover the depression of EPSC-amplitudes for large first amplitudes, shown in Fig. 2.6B (white rectangles).

In Fig. 2.6 C the theoretically predicted recovery after complete depletion of both pools is displayed. In accordance with the findings of Wu and Borst (1999) and with experimental results at the rat climbing fiber-Purkinje cell synapses (Silver et al., 1998) our calculations indicate that the joint occupancy of both pools recovers with a two-exponential time course (solid line in Fig. 2.6C). The initial rapid increase in the total number of occupied sites is due to the rapid replenishment of pool 1 with a fast time-constant $\tau_{P 1} \sim 0.3 \mathrm{~s}$, while the slow component in the time-course of recovery reflects the much slower shuffling of vesicles from pool 1 to pool 2 (time constant, $\tau_{P 2} \sim 5.2 \mathrm{~s}$; see also Fig. 2.7 and next section). The recovery from complete depletion as tested by a subsequent single test-AP occurs with a single exponential time course (time-constant $\tau \sim 4.76 \mathrm{~s}$ ), which is about three times faster than the time constant observed by $\mathrm{Wu}$ and Borst (1999), but in the same order of magnitude as the estimates by Silver et al. (1998) and the measured recovery from depletion by a $100 \mathrm{~Hz}$-stimulus train $(\tau \sim 4.92 \mathrm{~s})$ displayed in Figs. 2.5 and 2.6. Finally notice, that the small bump in the early recovery from $100 \mathrm{~Hz}$-stimulation is also seen in the model-calculations and, as demonstrated in the next section, arises as a combined effect of facilitation and "overshooting" of pool 1.

\subsection{Discussion}

We have proposed a model of vesicle release, recruitment and facilitation of release due to repetitive activity, which can account for the experimentally observed patterns of synaptic depression (and facilitation) at the calyx of Held. Our model considers two types of vesicles, an immediately-releasable pool of vesicles and an additional, more reluctantlyreleasable type of vesicles. These two types of vesicles do not only differ in their releaseprobability, but also regarding the time scales of replenishment. We discus in the following that this sequential arrangement of refilling of the two pools with two different time constants provides an intrinsic dynamic mechanism of activity induced extra-recruitment of vesicles.

A previous attempt to model depressing and steady-state behavior at the calyx of Held had to assume an activity-, i.e. $\mathrm{Ca}^{2+}$-dependent extra-recruitment of vesicles to compensate for the shortcomings of the simple depletion model, but failed to be confirmed experimentally (Weis et al., 1999; Wu and Borst, 1999). We have followed a different idea: Compared to the simple depletion model our approach considers an additional pool of reluctantly-releasable vesicles (pool 1), which are released with a lower releaseprobability than the immediately-releasable vesicles from pool $\mathbf{2}$.

At rest the majority of presynaptic release-sites is occupied with immediately-releasable vesicles. During repetitive stimulation this pool $\mathbf{2}$ is quickly emptied by a few stimuli (see Fig. 2.7 B). It is slowly refilled after (partial) depletion via pool $\mathbf{1}$, which first replenishes 

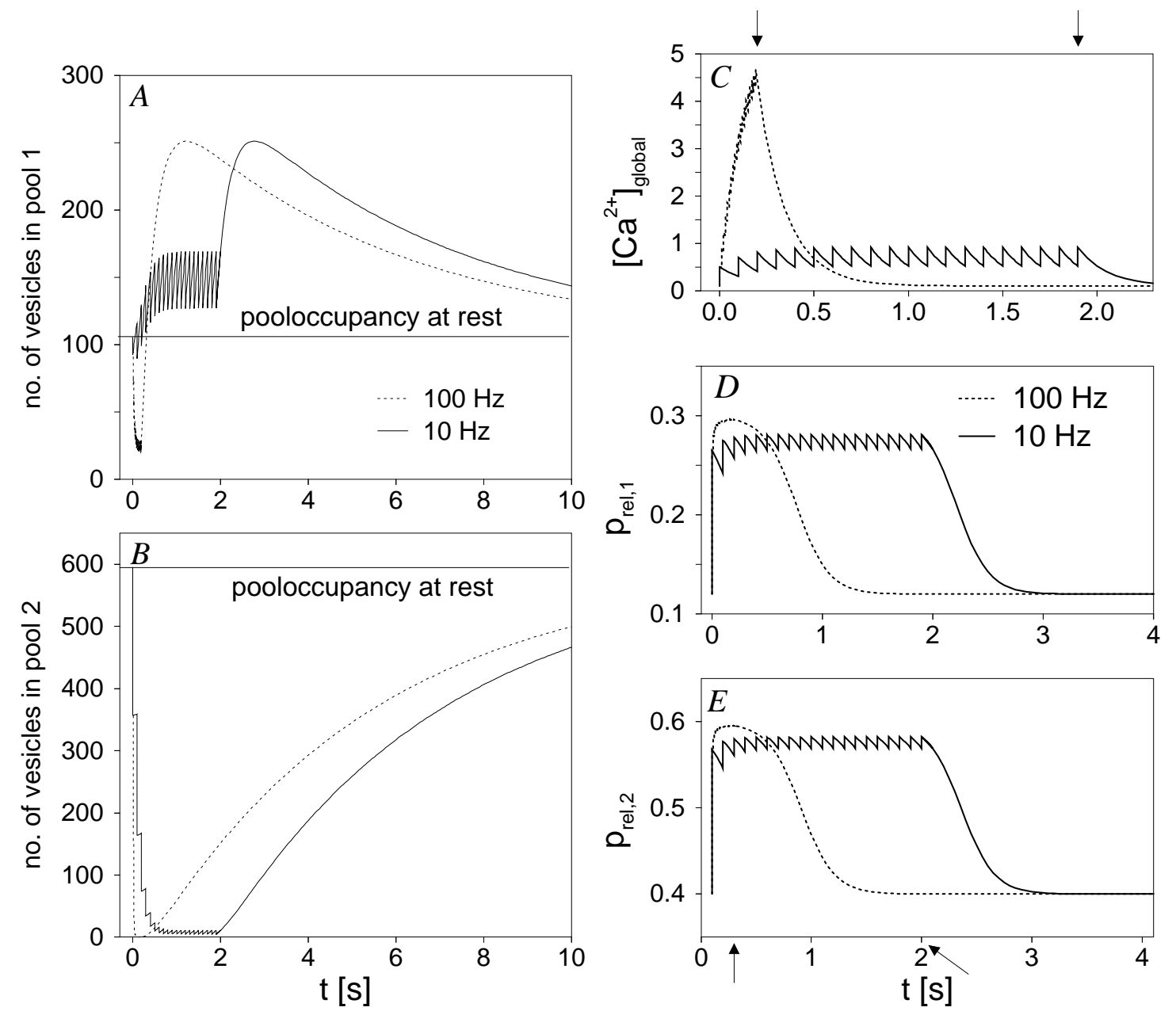

Figure 2.7.: Dynamics of vesicle recruitment and release as calculated from our model (parameters from set 2, Tab. 2.1) for stimulation with a frequency of $10 \mathrm{~Hz}$ (solid line) and $100 \mathrm{~Hz}$ (dashed line). A: Occupancy in pool $\mathbf{1}$ during and after stimulation. B: Occupancy in pool 2. $C$ : Changes in global presynaptic calcium concentration with repetitive stimulation as calculated by Eq. 2.16. D: Facilitation of release-probability $w_{1}$ due to repetitive stimulation (Eqs. 2.13). $E$ : Facilitation of release-probability $w_{2}$. The arrows indicate the end of the stimulus trains after 20 stimuli for stimulus frequencies of 10 and $100 \mathrm{~Hz}$. 
with a very fast time constant of $0.3 \mathrm{~s}$, before transitions to pool $\mathbf{2}$ occur on a much slower time scale $(\sim 5.2 \mathrm{~s})$.

Concerning pool 1 the situation is different. Once a sequence of APs has cleared most of the release-sites (which beforehand have been occupied to a large extent with immediatelyreleasable vesicles) those empty sites are quickly refilled, but due to the rapid transition between the reserve pool and pool 1 mostly with vesicles from pool $\mathbf{1}$. In other words, the depletion of pool $\mathbf{2}$ operates as a driving force on pool 1, causing an overshooting of the occupancy in pool $\mathbf{1}$ (compared to the occupancy at rest). This means, that pool $\mathbf{1}$ exhibits an intermediate occupancy higher than its value at rest (Fig. 2.7 A).

For stimulus frequencies low enough to allow an overshooting of pool $\mathbf{1}$ in between two subsequent stimuli, pool 1 already reaches a level of occupancy higher than rest during steady-state depression. This effect is illustrated in Fig. 2.7 $A$ for a $10-\mathrm{Hz}$ stimulus train, where the occupancy during steady-state depression is higher than at rest. The overshooting of pool 1 during steady-state depression is caused by the combined effects of repetitive depletion due to stimulation and rapid refilling within the inter-stimulus intervals.

For low stimulation frequencies $\left(<2 \mathrm{~Hz}\right.$ for $\left.\left[\mathrm{Ca}^{2+}\right]_{\text {out }}=2 \mathrm{mM}\right)$ the overshooting of pool 1 already decays towards the resting value before the next stimulus is applied, while for high stimulus frequencies the effect is impeded due to repeated depletion before recovery to resting values. Hence for a given frequency we expect a maximum overshooting of pool $\mathbf{1}$ during steady-state depression. This is confirmed in Fig. 2.8 $A$, where the occupancy of pool 1 during steady-state depression is shown as function of stimulation frequency. Maximum overshooting of pool $\mathbf{1}$ appears for a stimulation frequency of $2.3 \mathrm{~Hz}$ (at normal extracellular calcium, $\left[\mathrm{Ca}^{2+}\right]_{\text {out }}=2 \mathrm{mM}$ ). For higher release-probabilities, as caused by an increase in extracellular $\mathrm{Ca}^{2+}$, the frequency of maximum overshooting is shifted to lower frequencies, e.g. maximum at $1 \mathrm{~Hz}$ for $\left[\mathrm{Ca}^{2+}\right]_{\text {out }}=15 \mathrm{mM}$ (Fig. 2.8).

The overshooting of pool $\mathbf{1}$ is partly visible in the experimental data as the small bump for short time-intervals $\Delta t$ in the recovery from $100 \mathrm{~Hz}$-stimulation (Figs. 2.5 $A$ and 2.6 $A$ ): Immediately after the last stimulus the release-probabilities from both pools are still facilitated due to accumulation of residual calcium in the presynaptic terminal (Figs. 2.7 C, $D$ and $E$ ). This elevated probability interacts with the overshooting of pool $\mathbf{1}$. Hence a following test-AP causes an enhanced release out of pool $\mathbf{1}$ because the facilitated releaseprobability $w_{1}$ interacts with the overshooting occupancy in pool $\mathbf{1}$. The effect decays after approximately $1 \mathrm{~s}$ when $w_{1}$ has returned to resting-level and the "extra-occupancy" in pool $\mathbf{1}$ slowly replenishes pool 2 .

Our model yields the maximum of the small bump in the recovery at $\Delta t=0.5 \mathrm{~s}$ after the last stimulus in a $100 \mathrm{~Hz}$-train (arrow in Fig. 2.6 A). As displayed in Fig. 2.7 pool 1 is filled with $\sim 190$ vesicles (resting level $=105$ vesicles) at $\Delta t=0.5 \mathrm{~s}$ and is depleted by $w_{1} \sim 0.27$, whereas pool 2 contains only about 14 vesicles (corresponding releaseprobability $\left.w_{2} \sim 0.58\right)$. This implies, that the AP to test recovery is supplied to $\sim 86 \%$ by pool 1 .

However, when at $\Delta t \sim 1 \mathrm{~s}$ the occupancy of pool $\mathbf{1}$ is close to its maximum, only 

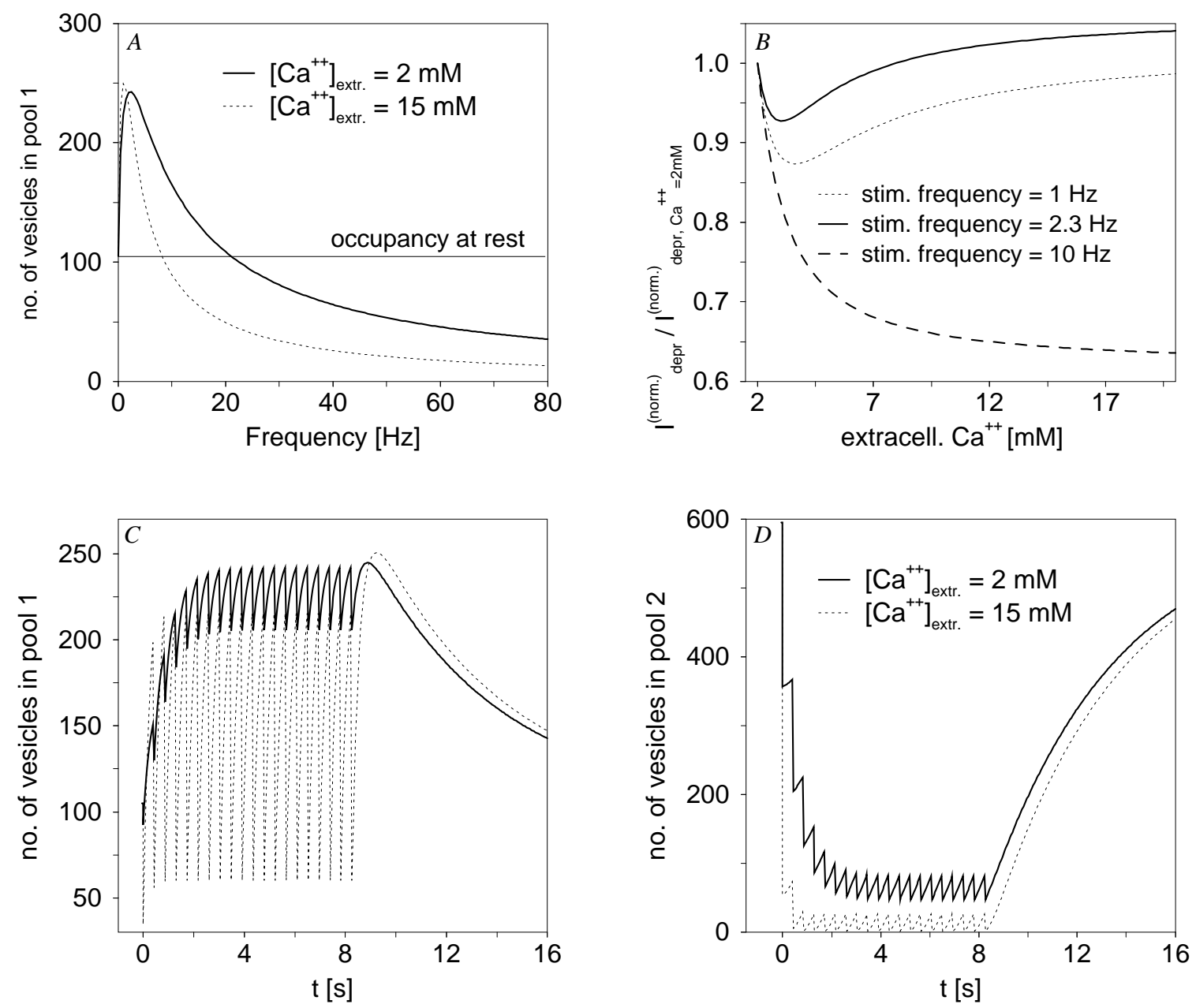

Figure 2.8.: Dependence of steady-state depression current on extracellular calcium, $\left[\mathrm{Ca}^{2+}\right]_{\text {out }}$. A: Steady-state depression occupancy in pool 1 as function of stimulation frequency for $\left[\mathrm{Ca}^{2+}\right]_{\text {out }}=2 \mathrm{mM}$ (solid line) and $\left[\mathrm{Ca}^{2+}\right]_{\text {out }}=15 \mathrm{mM}$ (dotted line). B: Normalized steady-state depression current (divided by normalized steady-state depression for $\left.\left[\mathrm{Ca}^{2+}\right]_{\text {out }}=2 \mathrm{mM}\right)$ as function of $\left[\mathrm{Ca}^{2+}\right]_{\text {out }}$ for three different stimulation frequencies: $1 \mathrm{~Hz}$ (dotted line), $2.3 \mathrm{~Hz}$ (solid line), and $10 \mathrm{~Hz}$ (dashed line). Stimulation with $2.3 \mathrm{~Hz}$ : Occupancy in pool 1 $(C)$ and pool $2(D)$ for $\left[\mathrm{Ca}^{2+}\right]_{\text {out }}=2 \mathrm{mM}$ (solid line) and $\left[\mathrm{Ca}^{2+}\right]_{\text {out }}=15 \mathrm{mM}$ (dotted line). 
$\sim 56 \%$ of the recovery is carried by release from pool 1 . At that time $w_{1}(\sim 0.14)$ has almost returned to resting level and the higher probability $w_{2}(\sim 0.44)$ causes a larger contribution from pool 2, which in the mean time has been refilled with $\sim 60$ vesicles. We conclude that the small bump seen in the recovery from depletion by high-frequency stimulation is a combined effect caused by facilitated release and overshooting of pool 1 .

We stress that our model is able to account for the patterns of short-term depression, without requiring $\mathrm{Ca}^{2+}$-dependent transition rates for pool-refilling. The dynamics of our model, arising from introducing two pools, their sequential arrangement and distinct timescales of refilling, contains an intrinsic mechanism of extra-recruitment, the overshooting of pool 1. We finally discuss how the predicted dynamics of pool $\mathbf{1}$ can be checked experimentally. As demonstrated above for stimulation-frequencies of $\sim 2 \mathrm{~Hz}$ the overshooting of pool 1 is already seen during steady-state depression. This behavior for low frequencies is displayed in Fig. 2.8C , where the increase in release-probabilities, e.g. by raising the extracellular calcium concentration, causes an enhanced release from pool 1 during steadystate depression compared to normal $\left[\mathrm{Ca}^{2+}\right]_{\text {out }}(=2 \mathrm{mM})$. In contrast the contribution of pool 2 to the steady-state depression decreases slightly for higher $\mathrm{Ca}^{2+}$-concentrations $\left(\sim 24\right.$ vesicles per stimulus at $\left[\mathrm{Ca}^{2+}\right]_{\text {out }}=15 \mathrm{mM}, \sim 35$ at $\left[\mathrm{Ca}^{2+}\right]_{\text {out }}=2 \mathrm{mM}$, see also Fig. 2.8D).

The combined effect of both pools leads to the characteristic change in the (normalized) steady-state depression current with changes in extra cellular calcium, which is demonstrated in Fig. 2.8B for three different stimulus frequencies. The plateau-like dependence of the (normalized) steady-state depression current on increasing extracellular calcium concentrations occurs only for frequencies which enable an overshooting of pool 1. For higher stimulus frequencies the steady state depression current decreases with higher extracellular $\mathrm{Ca}^{2+}$. In this case overshooting is repressed by repetitive pool-depletion, which is even more effective due to the overall enhanced release. This is also depicted in Fig. 2.8 $\mathrm{A}$, where not only the maximum overshooting of pool 1 is seen to shift to lower frequencies for higher extracellular $\mathrm{Ca}^{2+}$, but also the steady-state depression-occupancy in pool 1 already drops below resting level for stimulation frequencies of $\sim 9 \mathrm{~Hz}$.

We want to stress that the plateau-like shape of the (normalized) steady-state depression current for increasing extracellular calcium in our model depends on the extra-recruitment of vesicles into pool 1. Studying it experimentally could provide an additional validation of our model. We conclude that a heterogeneity in release probability, as e.g. observed in hippocampal connections (Rosenmund et al., 1993; Hessler et al., 1993; Murthy et al., 1997), may - in combination with specific time scales of replenishment — build the fundament of an intrinsic and activity-induced recruitment of vesicles.

In Chapter 5 we will utilize the results of this chapter to include presynaptic dynamics of vesicle recruitment, release and facilitation in our approach to study the transmission of information at central synapses. 


\section{Transmitter dynamics within the synaptic cleft}

In the previous chapter a model for presynaptic processes of vesicle recruitment and release has been derived. Our next step addresses the question how to calculate the change of transmitter concentration within the synaptic cleft after its release from a presynaptic vesicle.

Models of transmitter diffusion have been included in several theoretical approaches to modeling synaptic transmission at the neuromuscular junction (NMJ) (Agmon and Edelstein, 1997; Bartol et al., 1991; Bennett et al., 1997; Faber et al., 1992; Stiles et al., 1996) as well as at central synapses (Bartol et al., 1991; Busch and Sakmann, 1990; Holmes, 1995; Kleinle et al., 1996; Kruk et al., 1997; Marienhagen and Zippelius, 1995; Uteshev and Pennefather, 1996a; Uteshev and Pennefather, 1997; Wahl et al., 1996) . All these models generically involve free parameters which either have not been determined experimentally or are not accessible. For example the diffusion coefficient of glutamate has only been measured in aqueous solution (Longsworth, 1953) and not in the cleft. The values assigned to this parameter in various models of synaptic transmission vary from $10 \mathrm{~nm}^{2} / \mu \mathrm{s}$ (Kleinle et al., 1996) to $760 \mathrm{~nm}^{2} / \mu \mathrm{s}$ (Barbour et al., 1994), corresponding to its value in aqueous solution. Assumptions regarding mechanisms of transmitter release (see e.g., Kleinle et al., 1996) and uptake (see e.g., Holmes, 1995, Rusakov and Kullmann, 1998, Uteshev and Pennefather, 1997) also differ remarkably. Being aware of the large number of poorly known model parameters we design a theoretical model of diffusion within the synaptic cleft using the minimal number of free parameters necessary to account for the basic features of release from a vesicle, spreading of transmitter and uptake by transporter molecules or diffusion into extracellular space.

In the following we will estimate a net-diffusion coefficient $D_{\text {net }}$ to characterize the netdynamics of transmitter molecules, using information about the average residence time of transmitter molecules inside the cleft (Clements et al., 1992; Clements, 1996). We use the diffusion equation to model the net-movement of transmitter molecules inside the synaptic cleft, which is most likely slowed down compared to aqueous solution due to the tortuosity of the synaptic cleft (Garthwaite, 1985; Nicholson and Phillips, 1981; Rice et al., 1985; Ichimura and Hashimoto, 1988) or a cleft filled with dense staining, gellike material (Edwards, 1995a; Harris and Kater, 1994; Peters and Kaiserman-Abramof, 1969; den Loos, 1963). In the close vicinity of the cleft transporters assure rapid binding 
Transmitter dynamics within the synaptic cleft

of abundant transmitter (Asztely et al., 1997; Bergles and Jahr, 1997; Diamond and Jahr, 1997; Dzubay and Jahr, 1999; Edwards, 1995a; Isaacson and Nicoll, 1993; Mennerick et al., 1998; Otis et al., 1997; Takahashi et al., 1996; Tong and Jahr, 1994; Wang et al., 1998).

We will introduce a simple geometrical model of the cleft to comprise these features. We show that concentration fluctuations equilibrate rapidly across the height of the synaptic cleft, so that a two-dimensional profile is sufficient to account for spatial fluctuations in transmitter concentration. From the concentration profile of our model, we calculate the average residence time of transmitter molecules in the cleft and compare it to the experimentally observed time (Clements et al., 1992). This yields an estimate of the net-diffusion coefficient of $D_{\text {net }}=20-50 \mathrm{~nm}^{2} / \mu$ s.

\subsection{Diffusion of transmitter in the synaptic cleft}

As pre- and postsynaptic terminal stick together closely in central synapses, we model the synaptic cleft as a flat cylinder with height $h($ Fig. 3.1 $A)$. The transmitter is released from a point source at the presynaptic side of the cleft and spreads inside the cylindrical cleft according to Fick's second law. Certainly the transmitter movement inside the cleft is different from free diffusion in aqueous solution due to interactions with transporters or a dense, gel-like material (Edwards, 1995a; Harris and Kater, 1994). The effects of these interactions are twofold: First, frequent fast binding of transmitter to buffers or other molecules, as well as the tortuosity of the cleft, will slow down diffusion, but will not remove transmitter irreversibly from the cleft. Hence the transmitter dynamics remains diffusive for time scales relevant to the receptor kinetics and is modeled by a net diffusion coefficient $D_{\text {net }}$, smaller than the free diffusion coefficient $D_{\text {water }}$.

Second, uptake and transport into intracellular compartments causes depletion of transmitter. Although detailed information about the distribution and density of transporters in- and outside the cleft is not available so far, there seems to be agreement about the existence of intra- and extra-synaptic transporter mechanisms to ensure a rapid clearance of abundant transmitter molecules (Bergles and Jahr, 1997; Dzubay and Jahr, 1999; Hertz et al., 1978; Holmes, 1995; Kullmann and Asztely, 1998; Rusakov and Kullmann, 1998; Takahashi et al., 1996; Wang et al., 1998). We model neuronal and glial transmitter uptake by introducing an absorbing boundary for the diffusion field. Its location is chosen outside the postsynaptic density (PSD), which contains the postsynaptic receptors and typically exhibits a radius of $R=50$ to $200 \mathrm{~nm}$ (see e.g., Edwards, 1995b), so that the absorbing boundary is set in the range of $r_{\text {abs }}=500$ to $1000 \mathrm{~nm}$, i.e., comparable to the typical distance between neighboring synapses (Rusakov and Kullmann, 1998).

\footnotetext{
${ }^{1}$ There has been a discussion in the literature about the effect of diffusion pores on transmitter diffusion (Khanin et al., 1994; Holmes, 1995; Kleinle et al., 1996; Clements, 1996; Uteshev and Pennefather, 1996a). As the authors come to differing conclusions about the necessity of including a diffusion pore into theoretical modeling, we want to restrict our model to the simplest case for the beginning.
} 
$A$

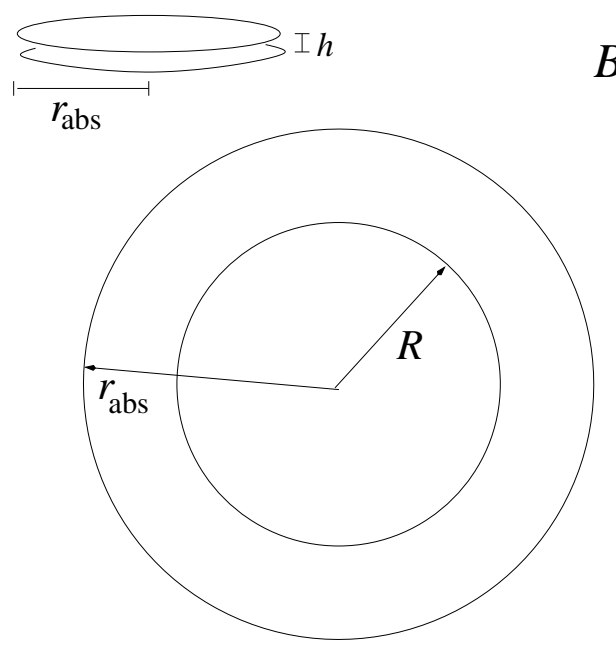

$B$

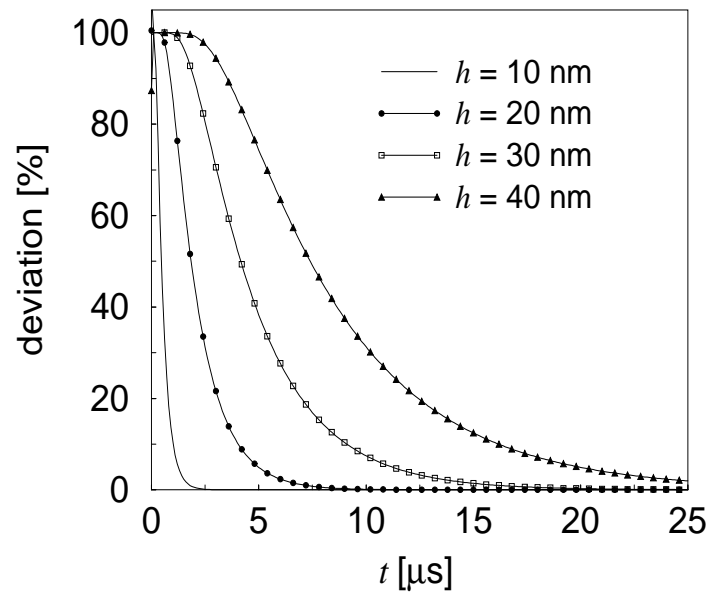

Figure 3.1.: A: Model of the synaptic cleft. The cleft is modeled by a flat cylinder of height $h$ and radius $r_{\text {abs. }}$. Postsynaptic receptors are distributed within the postsynaptic density (PSD) of radius $R$. Once the transmitter molecules hit $r_{\text {abs }}$ they are absorbed. $B$ : Difference $\Lambda$ between a two- and a threedimensional model of the synaptic cleft as function of time and for different heights $h$ of the synaptic cleft. The three-dimensional model is averaged over the small interval of height $\delta=5 \mathrm{~nm}$.

An absorbing boundary at larger distances has the same effect as less efficient uptake mechanisms.

\section{Computation of the diffusive concentration profile}

The spatio-temporal concentration profile $c(r, \varphi, z, t)$ of a number of $N_{T}$ transmitter molecules in the cleft is calculated by solving the three-dimensional diffusion equation

$$
\frac{\partial}{\partial t} c(r, \varphi, z, t)=D_{\text {net }}\left[\frac{\partial^{2}}{\partial r^{2}}+\frac{1}{r} \frac{\partial}{\partial r}+\frac{1}{r^{2}} \frac{\partial^{2}}{\partial \varphi^{2}}+\frac{\partial^{2}}{\partial z^{2}}\right] c(r, \varphi, z, t) .
$$

Since the synaptic cleft is chosen as a flat cylinder of height $h$ and radius $r_{\text {abs }}$ (Fig. 3.1 $A$ ) cylindrical coordinates $z, r, \varphi$ are used. The solution of the diffusion Eq. 3.1 is unique, provided we specify initial and boundary conditions. We assume that all molecules start at $t=t_{0}$ from a point at position $\mathbf{r}_{0}$, which is located somewhere on the upper lid of the cylindrical cleft corresponding to the release of a vesicle from the presynaptic terminal, i.e.,

$$
c(\mathbf{r}, t=0)=\frac{N_{T}}{r} \delta\left(r-r_{0}\right) \delta\left(\varphi-\varphi_{0}\right) \delta(z-h) .
$$


Diffusing molecules which reach the absorbing boundary at $r_{\mathrm{abs}}$ are taken out of the system. Transmitter molecules cannot escape in the $z$-direction, corresponding to a reflecting boundary at $z=0$ and $z=h$. These boundary conditions are summarized by the equations

$$
\begin{aligned}
& \text { reflecting boundary at } z=0, z=h:\left.\frac{\partial}{\partial z} c(\mathbf{r}, t)\right|_{z=0}=\left.\frac{\partial}{\partial z} c(\mathbf{r}, t)\right|_{z=h}=0 \\
& \text { absorbing boundary at } \quad r=r_{\mathrm{abs}}:\left.\quad c(\mathbf{r}, t)\right|_{r=r_{\mathrm{abs}}}=0 .
\end{aligned}
$$

Because of the specific form of boundary and initial conditions the solution of Eq. 3.1 separates into a one-dimensional probability distribution $p_{z}(z, t)$ to account for the distribution of molecules in $z$-direction and into a two-dimensional "lateral" probability distribution $p_{\Phi}(r, \varphi, t)$

$$
c(\mathbf{r}, t)=N_{T} p_{\Phi}(r, \varphi, t) p_{z}(z, t),
$$

with

$$
\begin{aligned}
p_{z}(z, t)= & \sum_{-\infty}^{\infty} \frac{(-1)^{n}}{h} \cos \left(\frac{n \pi z}{h}\right) \mathrm{e}^{-\frac{n^{2} \pi^{2}}{h^{2}} D_{\mathrm{net}} t}, \\
p_{\Phi}(r, \varphi, t)=-\frac{1}{r_{\mathrm{abs}}^{2} \pi} & {\left[\sum_{m=0}^{\infty} \sum_{n=1}^{\infty} \frac{J_{m}\left(\alpha_{m n} r_{0}\right)}{J_{m-1}\left(\lambda_{m n}\right) J_{m+1}\left(\lambda_{m n}\right)} J_{m}\left(\alpha_{m n} r\right) \mathrm{e}^{-\alpha_{m n}^{2} D_{\mathrm{net}} t}\right.} \\
& \left.\left\{\left[1+(-1)^{m}\right] \cos \left(m \varphi_{0}\right) \cos (m \varphi)+\left[1-(-1)^{m}\right] \sin \left(m \varphi_{0}\right) \sin (m \varphi)\right\}\right] .
\end{aligned}
$$

Here $J_{m}$ denotes the $m$ th Bessel function of the first kind, $\lambda_{m n}$ the $n$th zero of $J_{m}$ and $\alpha_{m n}=\lambda_{m n} / r_{\mathrm{abs}}$.

\subsection{Two-dimensional model of the synaptic cleft}

The extension $h$ of the synaptic cleft in the $z$-direction $(\sim 15-20 \mathrm{~nm})$ is more than ten times smaller than in lateral direction, so that it seems reasonable to reduce the model to a two-dimensional model of the synaptic cleft. To obtain a quantitative measure for the difference between two- and three-dimensional concentration profiles just above the PSD $(0 \leq z \leq \delta)$, we integrate the three-dimensional profile over a small interval $\delta$ (located right above the postsynaptic membrane at $z=0$ )

$$
c_{\delta}(r, \varphi, t)=N_{T} p_{\Phi}(r, \varphi, t) \int_{0}^{\delta} d z p_{z}(z, t)
$$

and compare it to the two-dimensional concentration profile, given by $c_{\Phi}(r, \varphi, t)=N_{T} p_{\Phi}(r, \varphi, t)$. 
The relative deviation $\Lambda$ is defined as

$$
\Lambda=\frac{c_{\Phi}(r, \varphi, t)-c_{\delta}(r, \varphi, t)}{c_{\Phi}(r, \varphi, t)}=-2 \frac{h}{\delta} \sum_{n=1}^{\infty} \frac{(-1)^{n}}{n \pi} \sin \left(\frac{n \pi \delta}{h}\right) \mathrm{e}^{-\frac{n^{2} \pi^{2}}{h^{2}} D_{\mathrm{net}} t}
$$

and shown in Fig. $3.1 B$ as a function of time for $\delta=5 \mathrm{~nm}$, different heights $h$ of the synaptic cleft and a relatively slow diffusion coefficient of $D_{\text {net }}=30 \mathrm{~nm}^{2} / \mu \mathrm{s}$. 2] The difference between a three- and a two-dimensional model of the synaptic cleft vanishes within a few microseconds. This indicates that we find a stationary state in the $z$-direction within microseconds, which is very fast compared to the time scale of receptor kinetics $(\sim \mathrm{ms})$, and hence rationalizes our approach to use a two dimensional model.

\subsection{Estimate of the diffusion coefficient}

By means of the spatio-temporal concentration profile $c_{\Phi}(r, \varphi, t)=N_{T} p_{\Phi}(r, \varphi, t)$ (Eq. 3.2) we will compute the residence time $\langle T\rangle$ of neurotransmitter in the cleft. Subsequently we will compare $\langle T\rangle$ to experimental findings for the synaptic geometry and the time-course of transmitter in the cleft to compute an estimate for the diffusion constant.

The residence time $\langle T\rangle$ of a particle, which diffuses along the path $X(t)$, is given as the integral over all times, which the particle spends inside the area $K_{R}=\pi R^{2}$ of the PSD, i.e.,

$$
\begin{aligned}
\langle T\rangle & =\int_{0}^{\infty} d t\left\langle\Upsilon_{K_{R}}(X(t))\right\rangle, \quad \Upsilon_{K_{R}}(x):= \begin{cases}1 & x \in K_{R} \\
0 & \text { elsewhere }\end{cases} \\
& =\frac{1}{N_{T}} \int_{0}^{\infty} d t \int_{0}^{2 \pi} d \varphi \int_{0}^{R} d r r c_{\Phi}(r, \varphi, t) \\
& =-\frac{1}{r_{\mathrm{abs}}^{2} \pi} \sum_{m=0}^{\infty} \sum_{n=1}^{\infty} \int_{0}^{\infty} d t \mathrm{e}^{-\alpha_{m n}^{2} D_{\mathrm{net}} t} \int_{0}^{R} d r r \frac{J_{m}\left(\alpha_{m n} r_{0}\right)}{J_{m-1}\left(\lambda_{m n}\right) J_{m+1}\left(\lambda_{m n}\right)} J_{m}\left(\alpha_{m n} r\right) \\
& \underbrace{\int_{0}^{2 \pi} d \varphi\left\{\left[1+(-1)^{m}\right] \cos \left(m \varphi_{0}\right) \cos (m \varphi)+\left[1-(-1)^{m}\right] \sin \left(m \varphi_{0}\right) \sin (m \varphi)\right\}}_{=4 \pi \delta_{0 n}} \\
& \frac{2 R r_{\mathrm{abs}}}{D_{\mathrm{net}}} \sum_{n=1}^{\infty} \frac{1}{\lambda_{0 n}^{3}} \frac{J_{0}\left(\alpha_{0 n} r_{0}\right) J_{1}\left(\alpha_{0 n} R\right)}{\left[J_{1}\left(\lambda_{0 n}\right)\right]^{2}}
\end{aligned}
$$

with $J_{m}$ denoting the $m$ th Bessel function of the first kind, $\lambda_{m n}$ the $n$th zero of $J_{m}$ and $\alpha_{m n}=\lambda_{m n} / r_{\text {abs }}$.

\footnotetext{
${ }^{2}$ Larger values of the diffusion constant give rise to an even faster decay of $\Lambda$.
} 
Equation 3.4 relates the mean residence time $\langle T\rangle$ to the radius $R$ of the PSD, the radius of the absorbing boundary $r_{\text {abs }}$ and the net-diffusion coefficient $D_{\text {net }}$. Experiments on glutamatergic synapses yield the following estimates: $\langle T\rangle \sim 1 \mathrm{~ms}$ for the residence time (Barbour et al., 1994; Clements et al., 1992; Spruston et al., 1995), $R \sim 200 \mathrm{~nm}$ for the radius of the PSD (see e.g., Edwards, 1995b) and $r_{\mathrm{abs}} \sim 500-1000 \mathrm{~nm}$ (Rusakov and Kullmann, 1998; Dzubay and Jahr, 1999). For an absorbing boundary set in the range $R \leq r_{\text {abs }} \leq 10 R$ we get $D_{\text {net }} \sim 20-50 \mathrm{~nm}^{2} / \mu$ s (Fig. 3.2).

If the range of transmitter diffusion is unbounded $\left(r_{\mathrm{abs}}=\infty\right)$, i.e., no uptake is considered at all (as done e.g., by Barbour et al., 1994, Kleinle et al., 1996, Wahl et al., 1996), the concentration profile is given exactly by $c_{\Phi}(r, \varphi, t)=N_{T} \exp \left(-r^{2} /\left(4 D_{\text {net }} t\right)\right) /\left(4 \pi D_{\text {net }} t\right)$. The mean residence time is then infinite due to a logarithmic divergence of the integral in Eq. 3.4 at long times and cannot be used to estimate $D_{\text {net }}$. Variations in the point of release do not contribute essentially (data not shown here), in contrast to differences in the extension of the PSD, which effect the estimate of $D_{\text {net }}$ (Fig. 3.2 $B$ ).
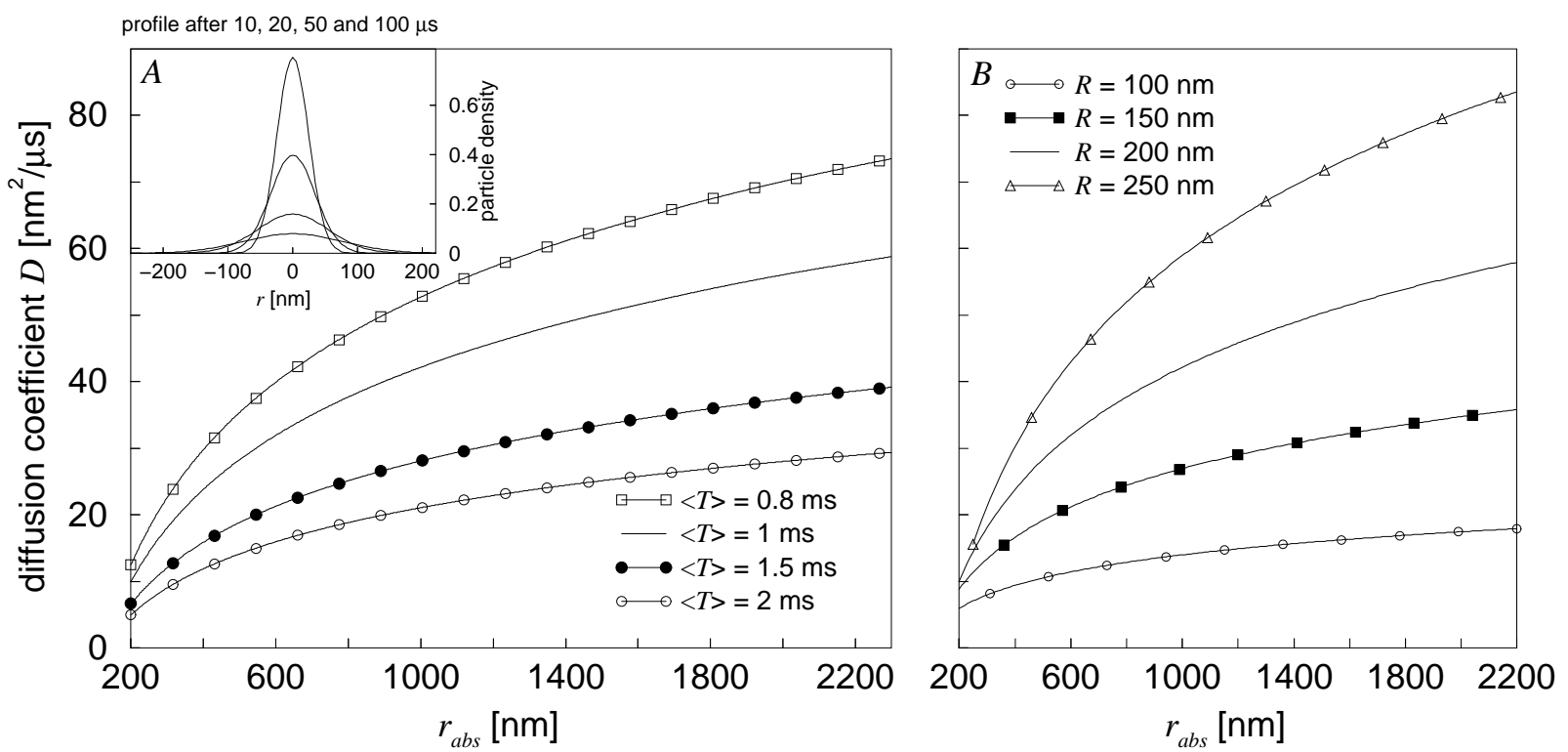

Figure 3.2.: Estimate of the net-diffusion coefficient $D_{\text {net }}$ as a function of the model parameter $r_{\text {abs. }}$ A: The spatio-temporal concentration profile (small upper inset) is averaged over time and area of the PSD. The diffusion coefficient $D_{\text {net }}$ is shown for $R=200 \mathrm{~nm}$ and several estimates of $\langle T\rangle$. Upper inset: Concentration profile as function of the radial component $r$ as calculated from Eq. 3.2, at times $t=10,20,50$ and $100 \mu \mathrm{s}$; transmitter release in the middle of the synaptic disc. $B$ : Estimate of $D_{\text {net }}$ for $\langle T\rangle=1 \mathrm{~ms}$ and different radii of the $\operatorname{PSD}(R=100,150,200$ and $250 \mathrm{~nm})$.

The calculated value of $D_{\text {net }}$ is one order of magnitude smaller than the free diffusion coefficient of glutamate $D_{\text {water }}=760 \mathrm{~nm}^{2} / \mu$ s in water (Longworth, 1953) and indicates 
that the transmitter molecules in the cleft are slowed down compared to free diffusion in aqueous solution. A reduction of the diffusion coefficient to a value of $D_{\text {net }} \sim 300 \mathrm{~nm}^{2} / \mu \mathrm{s}$ due to tortuosity of the cleft has been suggested (Garthwaite, 1985; Nicholson and Phillips, 1981; Rice et al., 1985; Ichimura and Hashimoto, 1988). Several theoretical approaches have followed this idea (Barbour et al., 1994; Holmes, 1995; Uteshev and Pennefather, 1997; Wahl et al., 1996). The reduction which we find $\left(D_{\text {net }} \sim 30 \mathrm{~nm}^{2} / \mu \mathrm{s}\right)$ is much stronger and in correspondence with Kleinle et al. (1996) and Bennett et al. (1997), who obtained reasonable time courses in calculated postsynaptic currents (EPSCs) using such a small diffusion coefficient as a free fit parameter.

Our intention was to keep the number of free model parameters low and the comparison to experiments as transparent as possible. We have proposed a simple description of the synaptic cleft as a flat disc with finite lateral extension. Transmitter molecules are allowed to diffuse up to a maximum distance, which is identified with the typical distance to neighboring synapses and which is modeled theoretically as an absorbing boundary. We emphasize that the estimated diffusion coefficient $D_{\text {net }}$ is independent of any kinetic receptor model, and is determined by the parameters $R, r_{\text {abs }}$ and $\langle T\rangle$ only. Within our approach we are able to estimate the net-diffusion coefficient $D_{\text {net }}$ of transmitter inside the cleft on the basis of experimentally known parameters only. We find $D_{\text {net }} \sim 20-50 \mathrm{~nm}^{2} / \mu \mathrm{s}$ for glutamate inside the cleft, implying a strong reduction as compared to free diffusion in aqueous solution. The parameter range is chosen to give an upper limit of the diffusion coefficient $D_{\text {net }}$, which still is about an order of magnitude smaller than the free diffusion coefficient.

\subsection{Outlook: Postsynaptic currents yield information about the transmitter time course in the cleft}

Postsynaptic responses can serve as indirect measurements of the transmitter transient in the cleft - provided the corresponding receptor kinetics is known from independent experiments as for instance patch-recordings (see Sec. 4.1). We will show in Chapter 4 that in this case the analysis of decay times or the distributions of decay times, respectively, allows additional conclusions about the residence time of transmitter in the cleft.

For example decay times of AMPA mediated EPSCs in hippocampal pyramidal cells (Jonas et al., 1993; Glavinovic, 1999), cerebellar Purkinje cells (Barbour et al., 1994; Häusser and Roth, 1997; Marienhagen et al., 1997) and brainstem interneurons (Chapter 4) are slower than the fast deactivation of AMPA channels (Sec. 4.1.2, see also Spruston et al., 1995, Jonas et al., 1993), so that desensitization contributes to shaping the time course of postsynaptic currents (Glavinovic, 1999; Häusser and Roth, 1997; Jonas et al., 1993; Jones and Westbrook, 1996; Spruston et al., 1995; Wahl et al., 1996). This means that glutamate has to be present in the cleft long enough to initiate receptor desensitization, which is in accordance with measurements indicating that glutamate remains in the synaptic cleft for almost a millisecond (Clements et al., 1992; Clements, 1996). A similar 
line of reasoning has been pursued in several experiments and simulations (Barbour et al., 1994; Diamond and Jahr, 1997; Holmes, 1995; Tong and Jahr, 1994), where it was shown that changes in the time course of glutamate, e.g. by block of glutamate uptake, lead to alterations in EPSC decay times. We therefore expect that decay times of EPSCs can provide additional information about the time course of glutamate in the cleft and in Sec. 4.6 will study distributions of decay times to trace the origin of synaptic variability back to heterogeneities in synaptic morphology affecting the transmitter time course in the cleft. 


\section{Studying the postsynaptic side}

In this chapter we will complete our collection of theoretical model building blocks of synaptic transmission by introducing a model of receptors in a single spine synapse (Fig. 1.2). We will explain how the theoretical analysis of miniature currents is able to provide information about receptor number, arrangement, saturation and synaptic morphology. We first will illustrate how receptors are modeled by Markov models, discuss two different kinetic models for glutamate activated AMPA receptors and demonstrate how the model parameters are extracted from experiments (Sec. 4.1). The current response of a population of postsynaptic receptors to the release of a single presynaptic vesicle, a so called miniature postsynaptic current (mEPSC), will be computed. This will be done using a complementary theoretical approach of Monte Carlo simulations (Sec. 4.2) and local chemical kinetics (Sec. 4.3) to calculate EPSCs. While Monte Carlo simulations preserve the typically observed noisy character of postsynaptic responses and hence allow an analysis of experimentally recorded distributions of amplitudes, rise and decay times, the "mean-field" approach of local chemical kinetics proves useful to study average quantities and possible effects due to a spatial arrangement of individual receptors.

We will carry out the analysis of miniature EPSCs for two different types of synapses to compare two types of fast excitatory glutamatergic synapses from two different parts of the brain with each other regarding their transmission properties. First, we will study experimental (Jonas et al., 1993) and simulated miniature distributions of hippocampal synapses. A wide range of experiments have been performed and a variety of models have been employed to understand the mechanisms of synaptic transmission in the hippocampus (Edwards, 1995a; Huang and Stevens, 1998; Malenka and Nicoll, 1999), which is thought to be crucially involved in storage of memory (Kandel et al., 1996). In particular it has been suggested that modifications in synaptic morphology can account for longterm changes in synaptic transmission (Edwards, 1995b; Engert and Bonhoeffer, 1999). We follow this idea and study how synaptic responses depend on spatial rearrangements of postsynaptic receptors and discuss possible mechanisms of long-term changes in synaptic efficacy (Sec. 4.5).

Second, we will analyze recordings from neurons of the nucleus tractus solitarius (NTS) in the brainstem (Titz and Keller, 1997), which exhibit extremely large fluctuations in the EPSC time course. We will demonstrate that the large fluctuations observed in miniature amplitudes are resolved by variations in the synaptic receptor number. In contrast the variability of EPSC decay times proves to be independent of fluctuating 
receptor numbers, but is explained by a heterogeneity in synaptic morphology affecting the transmitter motion (Sec. 4.6). This effect of the synaptic morphology does not occur in any other observable, suggesting that the analysis of the distribution of decay times yields additional insight in synaptic function.

\section{Miniature currents as the elementary transmission event}

To learn about postsynaptic features of synaptic transmission we choose to study so-called miniature synaptic currents (mEPSCs) - postsynaptic responses which result from the spontaneous release of a single vesicle (see Edwards, 1995b for review). Experiments generally yield a wide range of variability of these responses. Since mEPSCs are attributed to the release of a single vesicle they can be understood as elementary transmission events. Learning about the origin of their variability might elucidate basic mechanisms of synaptic function.

The large variability has predominantly been observed in the miniature amplitude distribution of postsynaptic currents (see Liu et al., 1999, Nusser et al., 1997 for further references) and its origin has been discussed controversially since. It has been suggested that variations in mEPSC amplitudes arise from presynaptic mechanisms such as transmitter release (Bekkers et al., 1990; Glavinovic, 1999; Forti et al., 1997; Frerking et al., 1995; Liu et al., 1999) or that they are caused by fluctuations located on the postsynaptic side as for instance variations in the receptor number (Borst et al., 1994; Edwards et al., 1990; Nusser et al., 1997; Tang et al., 1994).

Closely related to this discussion is the question whether synapses are saturated after the release of a single vesicle. It is widely believed that at central synapses an abundance of neurotransmitter hits a very limited number of postsynaptic receptors (see Edwards, 1995 and Walmsley, 1998 for reviews). Still, agreement regarding the question of saturation of postsynaptic receptors is lacking. Experimental and theoretical approaches have been chosen to address this question and have lead to the conclusion that the release of a single vesicle of glutamate activates most of non-NMDA receptors, i.e. causes an open probability larger than seventy percent (Jonas et al., 1993; Kleinle et al., 1996; Kullmann et al., 1999; Min et al., 1998; Silver et al., 1996; Spruston et al., 1995; Tang et al., 1994; Wahl et al., 1996). But also contradicting results, i.e. open probabilities of less than fifty percent, have been observed in mEPSCs (Liu et al., 1999; Mainen et al., 1999; Silver et al., 1996). The observed large fluctuations in mEPSC amplitudes are then are thought to be due to fluctuations in vesicle content (Harris and Sultan, 1995; Liu et al., 1999) or point of release (Uteshev and Pennefather, 1996a). These inconsistent findings for the open probability are resolved by the idea that receptor occupancy might be sensitive to several factors, such as synaptic morphology, the anatomy of the synaptic cleft, the time course of transmitter clearance, and that these properties most probably vary from one synapse to the next (Clements, 1996; Edwards, 1995a; Frerking and Wilson, 1996; Harris and Kater, 1994; Lim et al., 1999; Liu and Tsien, 1995; Min et al., 1998; Oleskevich et al., 1999; Rossi et al., 1995; Silver et al., 1996; Walmsley et al., 1998). 
Here we will address the question of origins of synaptic variability from a novel point of view by not only studying mechanisms that shape the distribution of amplitudes, but also those of rise and decay times. As pointed out in Sec. 3.4 decay times of AMPA mediated mEPSCs are slower than the fast deactivation of AMPA channels and we therefore expect that decay times of mEPSCs are partly determined by desensitization due to the prolonged presence of transmitter and can provide information about the time course of glutamate in the cleft.

\subsection{Receptor kinetics}

Single channel recording techniques (Sakmann and Neher, 1995) have been used to prove that transmitter activated currents arise from populations of individual ion channels undergoing rapid transitions between conducting and non-conducting states. The macroscopic behavior of the currents can be accurately captured using kinetic models that describe the transitions between conformational states of the receptors that gate the respective ion channels. These kinetic models belong to the class of so called Markov models: the transition probability into a new state depends only on the actual and the new state and is independent of previously accessed states. The models of receptors differ in their level of detail, ranging from the most detailed and biophysically realistic gating models to highly simplified representations. Models of transmitter mediated synaptic currents based on activation by brief increase in transmitter concentration after the release of a presynaptic vesicle should capture three aspects of receptor gating kinetics:

1. Activation/binding. The time course of the rising phase of the synaptic current is determined by the rate of opening after transmitter has been bound to the receptor. It is delayed, i.e. more sigmoidal, if more than one transmitter molecule has to be bound for the receptor to open.

2. Deactivation/unbinding: The time course of decay of postsynaptic currents is determined by either deactivation following transmitter removal or desensitization (see 3.). The rate of deactivation is limited either by the closing rate of the receptor or typically by the rate of unbinding of transmitter from the receptor.

3. Desensitization: Synaptic receptor-gated channels can be closed by entering a desensitized state, i.e. an in-activated, non-conducting state of the channel in which transmitter is bound to the receptor. Typically desensitization is slower than deactivation. 


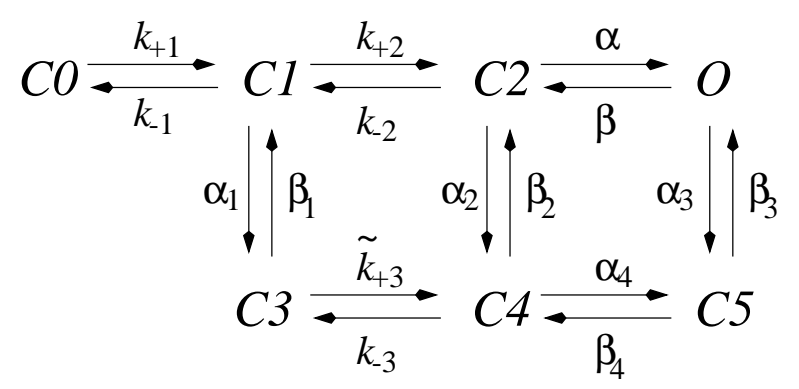

Figure 4.1.: Kinetic 7-state model of AMPA receptor (Jonas et al., 1993). The transitions marked by $\tilde{k}$ require the binding of a glutamate molecule. Kinetic rate constants are given by set 2 in (Jonas et al., 1993) as $\tilde{k}_{+1}=26.6 \mathrm{mM}^{-1} \mathrm{~ms}^{-1}, \quad k_{-1}=6.24 \mathrm{~ms}^{-1}, \quad \tilde{k}_{+2}=13.3 \mathrm{mM}^{-1} \mathrm{~ms}^{-1}$, $k_{-2}=12.5 \mathrm{~ms}^{-1}, \tilde{k}_{+3}=2.41 \mathrm{mM}^{-1} \mathrm{~ms}^{-1}, k_{-3}=0.057 \mathrm{~ms}^{-1}, \alpha=4.2 \mathrm{~ms}^{-1}$, $\beta=0.302 \mathrm{~ms}^{-1}, \alpha_{1}=0.513 \mathrm{~ms}^{-1}, \beta_{1}=0.0281 \mathrm{~ms}^{-1}, \alpha_{2}=0.395 \mathrm{~ms}^{-1}$, $\beta_{2}=0.546 \mathrm{~s}^{-1}, \alpha_{3}=0.109 \mathrm{~ms}^{-1}, \beta_{3}=0.0334 \mathrm{~ms}^{-1}, \alpha_{4}=0.00815 \mathrm{~ms}^{-1}$, $\beta_{4}=0.0103 \mathrm{~ms}^{-1}$.

\subsubsection{Kinetic seven-state model of AMPA receptors}

To undergo a transition into the open or desensitized state the AMPA receptor has to bind two glutamate molecules (Edmonds et al., 1995; Jahn et al., 1998). The kinetic scheme which is displayed in Fig. 4.1 comprises an unbound receptor state $(C 0)$, a single bound state $(C 1)$, a double bound state $(C 2)$, and an open state $(O)$, as well as corresponding desensitized states $(C 3, C 4, C 5)$.

This kinetic seven-state model of AMPA receptors has been derived on the basis of experiments at CA3 pyramidal cells in the hippocampus (Jonas et al., 1993) and has become a general standard to model AMPA receptors. The transition rate constants are gained from experimental estimates for receptor activation, deactivation, de- and resensitization as studied in outside-out patch recordings for varying glutamate concentrations (see below for further illustration of the experimental procedure). We will use this kinetic seven-state model of Jonas et al. (1993) in Sec. 4.5 to study sources of synaptic variability in hippocampal miniature currents.

The seven-state model, however, requires the knowledge of all 16 rate constants. In order to adapt the kinetic model to AMPA receptors in other areas of the brain we will introduce a simplified three-state model. Due to its simplicity the model avoids problems of ambiguity for the fit to experimental data, but still manages to comprise the basic features of the synaptic transmission process (Destexhe et al., 1994; Destexhe et al., 1998; Marienhagen et al., 1997; Uteshev and Pennefather, 1996b; Uteshev and Pennefather, 1997). 


\subsubsection{Kinetic three-state model of AMPA receptors}

Following the work of Marienhagen et al. (1997) the AMPA receptor is modeled using the kinetic three-state model defined by

$$
\mathrm{D} \underset{\tilde{k}_{d}}{\stackrel{k_{r}}{\rightleftarrows}} \mathrm{R} \underset{k_{c}}{\stackrel{\tilde{k}_{o}}{\rightleftarrows}} \mathrm{O} .
$$

Here $\mathrm{R}$ denotes the closed unbound, $\mathrm{O}$ the open conducting and $\mathrm{D}$ the desensitized state. As seen in the previous section the transition rates between the open state $\mathrm{O}$ and desensitized states are relatively small $\left(\alpha_{3}, \alpha_{4}, \beta_{3}\right.$ and $\beta_{4}$ in Fig. 4.1) and are neglected in this context for the sake of simplicity.

To undergo a transition into the open or desensitized state the receptor binds two glutamate molecules (Edmonds et al., 1995; Jahn et al., 1998), which is reflected by the transmitter dependency of the transition rates $\tilde{k}_{o}$ and $\tilde{k}_{d}$. In a first approximation the two binding steps of glutamate are assumed to be rapid compared to the time scales of channel opening and desensitizing (see also Häusser and Roth, 1997; Jahn et al., 1998; Jonas et al., 1993; Spruston et al., 1995). We assume that the glutamate binding reaction is at equilibrium compared to the time scales of channel opening and desensitization. The concentration dependency of $\tilde{k}_{o}\left(\tilde{k}_{d}\right.$, respectively) is then given by

$$
\tilde{k}_{o}=k_{o}[\mathrm{Glu}]^{2} /\left([\mathrm{Glu}]+K_{B}\right)^{2} \text {, }
$$

with $K_{B}$ denoting the dissociation constant (Colquhoun and Hawkes, 1995).

Assuming that $K_{B}$ is the same for activation and desensitization the kinetic behavior of the AMPA receptor is determined by the five constants $k_{r}, k_{d}, k_{o}, k_{c}$ and $K_{B}$. In the following we describe how they are estimated from experiments.

\subsubsection{Fit to experimental data: insight from outside-out patch recordings}

In general the study of central synapses is hampered by inaccessibility, rapid kinetics, the difficulty of measuring or controlling the time course of neurotransmitter, and the electronically remote location of synapses from somatic recording sites. Nevertheless, progress in understanding the gating of these receptors has been made through the fast perfusion of transmitter to excised membrane patches containing receptors (Kandel et al., 1996; Sakmann and Neher, 1995). In these patch-experiments a small piece of membrane is removed from the cell and kept in a small glass pipette. In the outside-out configuration the outside of the cell membrane is directed towards the outside of the patch-pipette. With this experimental set-up chemical solutions of known consistence can be applied to the receptors in the membrane patch and the initiated current through the membrane can be measured. Systematic variations in transmitter concentration, as well as its duration of 
A

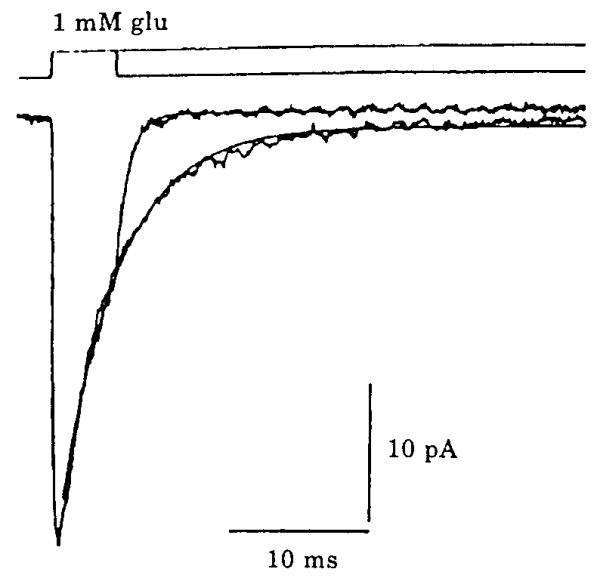

$B$

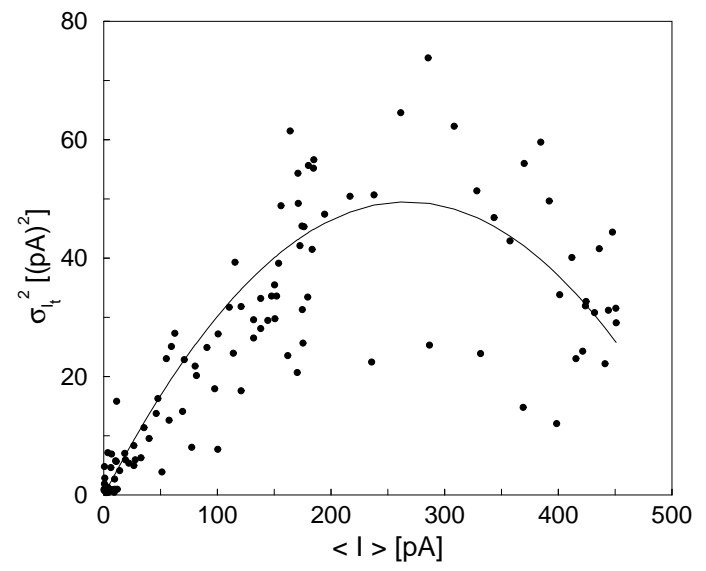

Figure 4.2.: A: Current responses of AMPA receptor channels in outside-out patches pulled from cerebellar Purkinje cells (taken from Barbour et al., 1994). B: Mean variance for a total of 10 responses obtained in outside-out patches of brainstem interneurons as a function of the mean current for all 10 responses. The data are fitted with Eq. 4.3 to determine $n_{\text {rec }}$ and $P_{\text {open,max }}$.

application are then used to study characteristics of rise, decay and desensitization in the receptor mediated current responses (Fig. 4.2 $A$ ).

As shown in Fig. 4.2 $\mathrm{A}$ for outside-out recordings from cerebellar Purkinje cells (Barbour et al., 1994) responses of AMPA receptors exhibit a very fast rise time in the submillisecond range. After the fast removal of transmitter the current decays within approximately $\sim 1 \mathrm{~ms}$. Desensitization of receptors in the permanent presence of transmitter occurs on a slower time scale of a few milliseconds. Recovery from desensitization is studied by application of two subsequent transmitter pulses, which yield a very slow time-constant of recovery of about 50 to 100 ms (Barbour et al., 1994; Häusser and Roth, 1997; Titz and Keller, 1997).

Patch-experiments do not only serve to study the different time scales of receptor activation or deactivation, but also yield information about the number of receptors activated at peak amplitude, i.e. about the maximum open probability $P_{\text {open,max }}$.

\subsubsection{Non-stationary fluctuation analysis}

The question of maximum activation is of interest concerning questions of postsynaptic receptor-saturation (see Sec. 4.5 and 4.6) and is addressed by means of non-stationary fluctuation analysis (Hille, 1992; Sigworth, 1980). The key idea here is to treat every individual receptor as a binary random variable. The two states of the variable are "conducting" and "non-conducting". Suppose there is only one channel which opens with 
the probability $P_{\text {open }}$ to pass a current $i_{S}$. If the current is recorded long enough for the channel to open many times, the mean current is $i_{S} P_{\text {open }}$ and the variance is given by

$$
\sigma^{2}=i_{S}^{2} P_{\text {open }}-i_{S}^{2} P_{\text {open }}^{2}
$$

As the membrane-patch is exposed to a spatially homogeneous transmitter concentration, each of the $n_{\text {rec }}$ receptors can be assumed to operate independently under the same conditions. Then the variances for the $n_{\text {rec }}$ independent and identical channels add, which yields the mean $\langle I\rangle$ and variance $\sigma_{I}^{2}$ of the total current $I$ :

$$
\langle I\rangle=n_{\text {rec }} i_{S} P_{\text {open }} \quad \text { and } \quad \sigma_{I}^{2}=n_{\text {rec }} i_{S}^{2} P_{\text {open }}-n_{\text {rec }} i_{S}^{2} P_{\text {open }}^{2} .
$$

Combining the two equations yields a quadratic relation between $\sigma_{I}^{2}$ and $\langle I\rangle$,

$$
\sigma_{I}^{2}=i_{S}\langle I\rangle-\frac{1}{n_{\text {rec }}}\langle I\rangle^{2}
$$

Here the open probability varies as function of time. In this case the mean current $\langle I\rangle$ and the variance $\sigma_{I}^{2}$ are computed within small time-intervals from an ensemble-average over many current time-courses. As shown in Fig. $4.2 B$ the membrane current is averaged over several measurements and the variance is computed within short time intervals. Then the variance is plotted as function of the corresponding mean current. A fit of Eq. 4.3 to the set of experimental data yields estimates of $i_{S}$ and $n_{\text {rec }}$. The maximum fraction of open channels can be computed by means of $i_{S}$ and $n_{\text {rec }}$ from the largest value of $\langle I\rangle$.

In Fig. $4.2 B$ the maximum open probability of AMPA receptors in brainstem outside-out patches which have been exposed to $1 \mathrm{mM}$ of glutamate is estimated using the method of non-stationary fluctuation analysis. The (time-dependent) average current $\langle I\rangle_{t}$ is calculated averaging 10 individual current responses from nucleated outside-out patches (Titz and Keller, 1997). To obtain the variance $\sigma_{I_{t}}^{2}$ every individual response is subtracted from the averaged current and the difference is squared. The 119 data points for $\sigma_{I_{t}}^{2}$ are then fitted by Eq. 4.3 for a single channel current $i$ of 0.372 pA (Titz and Keller, 1997). The fit (solid line in Fig. 4.2 $B$ ) yields an average number of $1431 \pm 24$ receptors $(68.3 \%$ confidence interval) in these large nucleated outside-out patches. This corresponds to a maximum open probability of $75 \%$ at peak amplitudes of $420 \mathrm{pA}$ (Fig. 4.2B).

\subsubsection{Estimate of kinetic rate constants from experiments}

The kinetic parameters of reaction scheme (4.1) are estimated by calculating the open probability $P_{\mathrm{O}}(t)$ as described in Marienhagen et al. (1997). In their work the timeconstant of desensitization has been computed as $\tau_{\text {des }}=\tau_{+}$, with

$$
\frac{1}{\tau_{ \pm}}=\frac{k_{c}+k_{r}+\tilde{k}_{o}+\tilde{k}_{d}}{2} \mp \sqrt{\frac{1}{4}\left(k_{c}-k_{r}+\tilde{k}_{o}-\tilde{k}_{d}\right)^{2}+\tilde{k}_{o} \tilde{k}_{d}} .
$$




\begin{tabular}{lc} 
observable & experimental estimate \\
\hline desensitization time constant & $\tau_{\text {des }}(1 \mathrm{mM})=5.47 \pm 1.3 \mathrm{~ms}$ \\
deactivation time constant & $\tau_{\text {dea }}=0.8 \pm 0.3 \mathrm{~ms}$ \\
resensitization time & $\tau_{\text {res }}=50 \pm 12 \mathrm{~ms}$ \\
maximum open probability & $P_{\mathrm{O}, \max }([1 \mathrm{mM}])=75 \%$ (Fig. $\left.4.2 B\right)$ \\
\hline
\end{tabular}

Table 4.1.: Kinetics of AMPA receptor as estimated from rapid application of glutamate to outside-out patches in brainstem interneurons (Titz and Keller, 1996; Titz and Keller, 1997).

In our approach the concentration-dependency of the transition rates $\tilde{k}_{o}$ and $\tilde{k}_{d}$ is given by Eq. 4.2 .

Furthermore the maximum open probability $P_{\mathrm{O}, \max }$ has been calculated as

$$
P_{\mathrm{O}, \max }=p_{o}^{s s}+\left[\mathrm{e}^{-t_{\max } / \tau_{-}}\left(1-k_{r} \tau_{-}\right)-\mathrm{e}^{-t_{\max } / \tau_{+}}\left(1-k_{r} \tau_{+}\right)\right] \frac{\tilde{k}_{o}}{1 / \tau_{+}-1 / \tau_{-}},
$$

with

$$
t_{\max }=\frac{\tau_{+} \tau_{-}}{\tau_{+}-\tau_{-}}\left(\ln \frac{\tau_{+}}{\tau_{-}}+\ln \frac{1-k_{r} \tau_{-}}{1-k_{r} \tau_{+}}\right) \quad \text { and } \quad p_{o}^{s s}=\frac{k_{r} \tilde{k}_{o}}{\left(k_{r}+\tilde{k}_{d}\right)\left(k_{c}+\tilde{k}_{o}\right)-\tilde{k}_{o} \tilde{k}_{d}} .
$$

The complete characterization of the receptor dynamics requires the knowledge of the kinetic rate constants $k_{i}(i=c, d, o, r)$ and of the equilibrium constant $K_{B}$, which subsequently are estimated by identifying the theoretical expressions with the results of outsideout patch recordings. The rate constant $k_{c}$ is given as the inverse of the deactivation time $\tau_{\text {dea }}$, i.e. $k_{c}=1 / \tau_{\text {dea }}$, and similarly the rate of resensitization $k_{r}$ by $1 / \tau_{\text {res }}$. The remaining two rates $k_{o}$ and $k_{d}$, as well as the equilibrium constant $K_{B}$ are computed by simultaneously fitting Eq. 4.4 for a known transmitter concentration to the experimentally determined time constant of desensitization and Eq. 4.5 to the maximum open probability $P_{\mathrm{O}, \max }$.

As explained in the previous section outside-out recordings at brainstem interneurons (Titz and Keller, 1996; Titz and Keller, 1997) provide an estimate for the maximum open-probability of $P_{\mathrm{O} \text {,max }}=75 \%$. The experimental findings for $\tau_{\text {dea }}, \tau_{\text {res }}$ and $\tau_{\text {des }}$ are summarized in Tab. 4.1. The simultaneous fit of $\tau_{\text {des }}(1 \mathrm{mM})$ (Eq. 4.4) and $P_{\mathrm{O}, \max }([1 \mathrm{mM}])$ (Eq. 4.5) yields the kinetic rate constants in Tab. 4.2 which will be used in the Monte Carlo simulations of brainstem interneurons to model the individual AMPA receptor. The computed equilibrium constant $K_{B}=450 \mu \mathrm{M}$ is in the same order of magnitude as the $\mathrm{EC}_{50}$ estimates for half-maximal activation given by Hässer and Roth (1997) for cerebellar Purkinje cells $\left(\mathrm{EC}_{50}=431 \mu \mathrm{M}\right)$ and by Jonas et al. (1993) for hippocampal CA3 cells $\left(\mathrm{EC}_{50}=355 \mu \mathrm{M}\right)$.

For the simulations of AMPA receptors in hippocampal synapses we will use the sevenstate model introduced in Sec. 4.1.1 and employ estimates from the literature (Jonas et al., 1993); values of the respective rate constants are shown in the figure caption of Fig. 4.1. 


\begin{tabular}{|c|c|c|c|c|}
\hline$k_{o}$ & $k_{d}$ & $k_{c}$ & $k_{r}$ & $\mathrm{~K}_{\mathrm{B}}$ \\
\hline $6 \mathrm{~ms}^{-1}$ & $1.1 \mathrm{~ms}^{-1}$ & $1.25 \mathrm{~ms}^{-1}$ & $0.02 \mathrm{~ms}^{-1}$ & $450 \mu \mathrm{M}$ \\
\hline
\end{tabular}

Table 4.2.: Kinetic rate constants for the AMPA receptor

The rate constants for the two kinetic models of AMPA receptors introduced here are estimated from patch-experiments where the applied transmitter concentration is assumed to be approximately spatially homogeneous for a known time window. This is not the situation in the synaptic cleft after the release of transmitter from a presynaptic vesicle. Shortly after the release from a vesicle the transmitter concentration peaks around the point of release and subsequently spreads within the cleft as calculated in Chapter 3 and illustrated in Fig. 3.2. In the simple spine synapse a limited number of individual receptors is distributed across the postsynaptic density (PSD) and due to varying distances from the point of release each receptor faces a different (time-dependent) local transmitter concentration. Our combined approach of Monte Carlo simulations and local chemical kinetics tries to map this scenario and is explained in the following.

\subsection{Monte Carlo simulations of miniature EPSCs}

Monte Carlo simulations of a postsynaptic receptor population exposed to transmitter released from a single vesicle are designed following Bartol et al. (1991) and Wahl et al. (1996). A number of $n_{\text {rec }}$ individual AMPA receptors are placed at random positions $\mathbf{r}_{i}\left(i=1, \ldots, n_{\mathrm{rec}}\right)$, uniformly distributed across the PSD. Instead of describing the change in transmitter concentration by the concentration profile derived in Sec. 3.1 we compute individual stochastic trajectories of transmitter molecules. In contrast to most Monte Carlo studies, which treat diffusion on a grid model of the cleft (Agmon and Edelstein, 1997; Bartol et al., 1991; Bennett et al., 1995; Bennett et al., 1997; Faber et al., 1992; Kruk et al., 1997; Stiles et al., 1996) we chose a continuous model of diffusion steps for discrete time steps $\Delta t$. As explained in Chapter 3 all transmitter molecules are released at once from a point source. The subsequent diffusion steps of single transmitter molecules are then given by a Langevin equation (Gardiner, 1983) for the position $\mathbf{r}_{j}\left(t_{k}\right)=$ $\left(x_{j}\left(t_{k}\right), y_{j}\left(t_{k}\right)\right)$ of the $j$ th molecule at time $t_{k}$

$$
\begin{aligned}
& x_{n}\left(t_{i+1}\right)=x_{n}\left(t_{i}\right)+\eta_{1}\left(t_{i}\right) \sqrt{2 D \Delta t}, \\
& y_{n}\left(t_{i+1}\right)=y_{n}\left(t_{i}\right)+\eta_{2}\left(t_{i}\right) \sqrt{2 D \Delta t},
\end{aligned}
$$

where $\eta_{1}\left(t_{i}\right)$ and $\eta_{2}\left(t_{i}\right)$ denote Gaussian distributed random numbers with

$$
\langle\eta(t)\rangle=0 ; \quad\left\langle\eta_{i}(t) \eta_{j}\left(t^{\prime}\right)\right\rangle=\delta_{i j} \delta\left(t-t^{\prime}\right) .
$$

As shown in detail in Bartol et al. (1991) statistical averages can either be calculated by averaging over many possible diffusion paths or equivalently by using the spatio-temporal concentration profile $c_{\Phi}(r, \varphi, t)$, as calculated in Sec. 3.1. 
For a given distribution of transmitter molecules the receptors are updated in fixed order by calculating the transition probabilities $k_{j} \Delta t(j=+1,-1$, etc.) for the respective accessible states and comparing them to a random number. In order to model AMPA receptors at hippocampal pyramidal cells we use the kinetic seven-state model (Fig. 4.1) by Jonas et al. (1993) as introduced in Sec. 4.1.1. When studying recorded miniature currents at brainstem interneurons we employ the three-state model of AMPA receptors as defined in Sec. 4.1.2 with the corresponding transition probabilities per unit time given in terms of the rates in Tab. 4.2.

Transitions between some of the receptor states require the binding of glutamate molecules. In the simplest approximation, this process is modeled by transition rates which depend on the local transmitter concentration. In the seven-state model (Fig. 4.1) these are: $\tilde{k}_{+1}, \tilde{k}_{+2}$, and $\tilde{k}_{+3}$. To estimate the local transmitter concentration we count the number of molecules inside a disc of "binding-radius" $r_{\text {bind }}^{j}$ around the $j$ th receptor. The concentration in units of $\mathrm{mM}$ is given by dividing this number by the small volume element $\Delta V=h \pi\left(r_{\text {bind }}^{j}\right)^{2}$. For instance the transition rate of the $j$ th receptor to make a transition from $C 0$ to $C 1$ is computed as

$$
\tilde{k}_{+1}^{(j)}=\frac{\left(\begin{array}{l}
\text { no of molecules inside } \\
\text { disc of radius } r_{\text {bind }}^{j}
\end{array}\right) k_{+1}}{\left(r_{\text {bind }}^{(j)}\right)^{2} \pi h N_{A}},
$$

with $h$ denoting the height of the synaptic cleft and $N_{A}$ Avogadro's number. If a transition into the states $C 1, C 2$ (from $C 1$ ) or $C 4$ (from $C 3$ ) occurs, the receptor binds a transmitter molecule, which is being released if the back-transition follows. It should be noted here that the parameter $r_{\text {bind }}$ is necessary to determine the local concentration. We checked that the results discussed further down do not depend on the specific choice of $r_{\text {bind }}$, which in our simulations was set to $6 \mathrm{~nm}$.

For each discrete time step $\Delta t$ the concentration profile of neurotransmitter and the states of all receptors are modeled as follows:

1. A new distribution of transmitter molecules is generated according to Eq. 4.6.

2. The "local" number of transmitter molecules in the vicinity of every individual receptor is computed and the individual transition probabilities are calculated by Eq. 4.7 with the kinetic rate constants of Fig. 4.1.

3. The states of all receptors are updated by comparing the individual transition probabilities to a random number.

4. The number of receptors in the open state is counted.

This yields the number of open channels or the open probability, respectively, as a function of time. For a series of sweeps, which are run for a set of given synaptic parameters but varying initializations of the random number generator, distributions of amplitudes, rise 
and decay times are generated and compared to recordings of mEPSCs. The coefficient of variation $(\mathrm{CV}$ - standard deviation/mean) is taken as an index of variability.

The computer simulations have been written in C language, compiled and run on Pentium PCs. Random numbers are generated using the ran2 routine (Press et al., 1992). Tabular 4.3 contains the values, which in the simulations have been assigned to the model parameters. The simulation time step is chosen to be $4 \mu \mathrm{s}$, which is considerably smaller than the fastest kinetic rate constant of channel opening and fast enough to account for the diffusion of transmitter molecules.

Table 4.3.: Parameters as used in the Monte Carlo simulations of a receptor population in a simple spine synapse in the hippocampus.

\begin{tabular}{|c|c|c|c|}
\hline Symbol & Definition & Value & Comment \\
\hline $\begin{array}{l}\text { Time ster } \\
\Delta t\end{array}$ & time step & $4 \mu \mathrm{s}$ & \\
\hline \multicolumn{4}{|l|}{ Geometry } \\
\hline$h$ & height synaptic cleft & $15 \mathrm{~nm}$ & from Edwards, 1995b \\
\hline$R$ & radius $\mathrm{PSD}$ & $50-400 \mathrm{~nm}$ & from Edwards, 1995 \\
\hline$r_{\mathrm{abs}}$ & $\begin{array}{l}\text { radius absorbing } \\
\text { boundary }\end{array}$ & $200-2000 \mathrm{~nm}$ & $\begin{array}{l}\text { from Rusakov and } \\
\text { Kullmann, } 1998\end{array}$ \\
\hline$\Delta F$ & binding area & $113.1 \mathrm{~nm}^{2}$ & $\begin{array}{l}\text { free parameter, } \\
\text { kinetic model }\end{array}$ \\
\hline$r_{\text {bind }}$ & "binding radius" & $6 \mathrm{~nm}$ & $\begin{array}{l}\text { free parameter, } \\
\text { Monte Carlo simulatic }\end{array}$ \\
\hline \multicolumn{4}{|c|}{ Transmitter } \\
\hline$N_{T}$ & $\begin{array}{l}\text { no. of molecules } \\
\text { per vesicle }\end{array}$ & $2000-4000$ & from Edwards, 1995b \\
\hline$T_{\exp },\langle T\rangle$ & Time constant & $0.8-2 \mathrm{~ms}$ & from Clements, 1992 \\
\hline
\end{tabular}

Kinetic rate

constants

AMPA-receptor see Fig. 4.1 set 2 from Jonas et al., 1993 


\subsection{Receptor dynamics calculated by local chemical kinetic equations}

In the following we present a model to calculate postsynaptic EPSCs using chemical kinetic (master) equations, which explicitly account for the spatial distribution of individual receptors on the postsynaptic membrane. We model an ensemble of many spatially distributed receptors, each characterized by a set of probabilities to be in one of its accessible states. In addition, each receptor is exposed to a different concentration of glutamate which determines its individual transition rates.

Following (Land et al., 1981; Land et al., 1984) EPSCs are commonly calculated by kinetic rate equations under the assumption that each receptor in the postsynaptic density "sees" approximately the same glutamate concentration (Bartol et al., 1991; Holmes, 1995; Kleinle et al., 1996; Uteshev and Pennefather, 1997). Then it is sufficient to solve one set of kinetic equations (representing the average over all receptors). The transition rates are determined by the spatially averaged glutamate concentration. The fraction of open channels is obtained by multiplying with the total number of receptors in the PSD. For a given time $t$ the partial differential Eq. 3.1 is first integrated numerically. The resulting concentration profile is then averaged over the PSD and used in a set of kinetic master equations which are solved subsequently (Bartol et al., 1991; Holmes, 1995; Kleinle et al., 1996; Uteshev and Pennefather, 1997). Numerical procedures to solve partial differential equations as Eq. 3.1 are costly and limit the application of this approach (Bartol et al., 1991).

We briefly explain our approach. Instead of following the stochastic transitions of $n_{\text {rec }}$, as done in the Monte Carlo simulation, we may alternatively consider the joint probability distribution, to find receptor 1 in state $s_{1}$, receptor 2 in state $s_{2}, \ldots$ receptor $n$ in state $s_{n}$. This description in terms of probabilities in general involves $n_{\text {rec }}$ interacting receptors and is completely equivalent to the stochastic dynamics as modeled by the Monte Carlo simulations, as far as averaged quantities are concerned (Gardiner, 1983). Such $n$ particle distribution functions are however difficult to treat analytically or numerically. In our model the interaction among receptors is weak, and only arises from the competition of receptors for neurotransmitter which is abundant at central synapses. If we ignore this interaction, i.e. assume that the number of transmitter molecules temporarily bound to receptors is small compared to the total number, then the distribution for $n_{\text {rec }}$ receptors factorizes and we can solve the master equations for each receptor separately.

Note however that each receptor $i$ at a given position $\mathbf{r}_{i}$ "sees" a time-dependent local transmitter concentration $C_{i}^{(\Delta F)}(t)$, which explicitly depends on the position of the receptor and is obtained by integrating $c(\mathbf{r}, t)$ over the small area increment $\Delta F$ around $\mathbf{r}_{i}$ shown in Fig. 4.3. In the simplest case the transmitter molecules are released in the middle of the synaptic disc, so that the spatio-temporal concentration profile from Eq. 3.2 


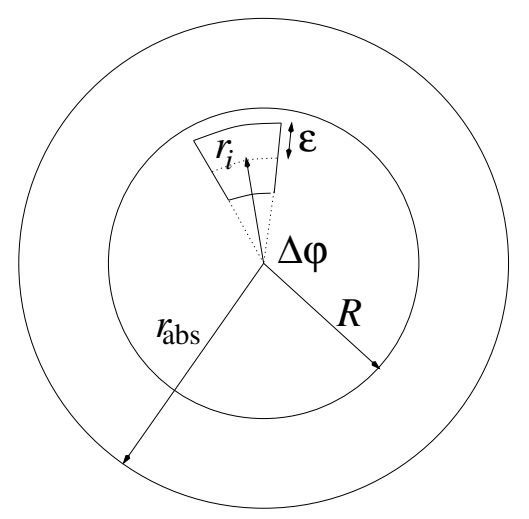

Figure 4.3.: Model of the synaptic cleft: Postsynaptic receptors are distributed within the postsynaptic density (PSD) of radius $R$. Once the transmitter molecules hit $r_{\text {abs }}$ they are absorbed. Small area increment $\Delta F=2 \varepsilon r_{i} \Delta \varphi=(2 \varepsilon)^{2}$, bounded by $\Delta \varphi$ and $2 \varepsilon$ to estimate the local transmitter concentration for a receptor located at position $\mathbf{r}_{i}$.

simplifies to

$$
c(\mathbf{r}, t)=\frac{N_{T}}{\pi r_{\text {abs }}^{2}} \sum_{n=1}^{\infty} \frac{J_{0}\left(\alpha_{0 n}|\mathbf{r}|\right)}{\left[J_{1}\left(\lambda_{n}\right)\right]^{2}} \mathrm{e}^{-\alpha_{0 n}^{2} D_{\text {net }} t}
$$

Integrating over $\Delta F$ (Fig. 4.3) yields for the local concentration $C_{i}^{(\Delta F)}(t)$

$$
C_{i}^{(\Delta F)}(t)=\frac{N_{T}}{r_{\mathrm{abs}}^{2}} \frac{\Delta \varphi}{\pi} \sum_{n=1}^{\infty} \frac{\left(r_{i}+\varepsilon\right) J_{1}\left[\alpha_{n}\left(r_{i}+\varepsilon\right)\right]-\left(r_{i}-\varepsilon\right) J_{1}\left[\alpha_{n}\left(r_{i}-\varepsilon\right)\right]}{\alpha_{n}\left[J_{1}\left(\lambda_{n}\right)\right]^{2}} \mathrm{e}^{-\alpha_{n}^{2} D_{\mathrm{net}} t}
$$

The local concentration in $m M$ then determines the transition rates according to円

$$
\tilde{k}_{i}=k_{i} \frac{C_{i}^{(\Delta F)}(t)}{\Delta F h N_{A}}
$$

As in the Monte Carlo model (Sec. 4.2) we assume that the kinetic rates $\tilde{k}_{+1}^{(i)}, \tilde{k}_{+2}^{(i)}$, and $\tilde{k}_{+3}^{(i)}$ of receptor $i$ at position $\mathbf{r}_{i}$ are proportional to the local, time-dependent transmitterconcentration. Hence for every individual receptor $i$ we have to solve the following set of seven coupled linear differential equations with time-dependent coefficients $C_{i}^{(\Delta F)}(t)$ in Eq. 4.8, which describe the dynamic evolution of the probabilities $P_{\alpha}^{i}$ for receptor $i$ to be

\footnotetext{
${ }^{1}$ The explicit choice of $\Delta F$ is not crucial for the calculated results (data not shown).
} 
in state $\alpha$ :

$$
\begin{aligned}
\frac{d}{d t} P_{C 0}^{(i)} & =-\tilde{k}_{+1} P_{C 1}^{(i)}+k_{-1} P_{C 2}^{(i)} \\
\frac{d}{d t} P_{C 1}^{(i)} & =\tilde{k}_{+1} P_{C 0}^{(i)}-\left\{k_{-1}+\tilde{k}_{+2}+\alpha_{1}\right\} P_{C 1}^{(i)}+k_{-2} P_{C 2}^{(i)}+\beta_{1} P_{C 3}^{(i)} \\
\frac{d}{d t} P_{C 2}^{(i)} & =\tilde{k}_{+2} P_{C 1}^{(i)}-\left\{k_{-2}+\alpha+\alpha_{2}\right\} P_{C 2}^{(i)}+\beta P_{O}^{(i)}+\beta_{2} P_{C 4}^{(i)} \\
\frac{d}{d t} P_{C 3}^{(i)} & =\alpha_{1} P_{C 1}^{(i)}-\left\{\beta_{1}+\tilde{k}_{+3}\right\} P_{C 3}^{(i)}+k_{-3} P_{C 4}^{(i)} \\
\frac{d}{d t} P_{C 4}^{(i)} & =\tilde{k}_{+3} P_{C 3}^{(i)}+\alpha_{2} P_{C 2}^{(i)}-\left\{k_{-3}+\beta_{2}+\alpha_{4}\right\} P_{C 4}^{(i)}+\beta_{4} P_{C 5}^{(i)} \\
\frac{d}{d t} P_{C 5}^{(i)} & =\alpha_{4} P_{C 4}^{(i)}-\left\{\beta_{4}+\beta_{3}\right\} P_{C 5}^{(i)}+\alpha_{+3} P_{O}^{(i)} \\
\frac{d}{d t} P_{O}^{(i)} & =\alpha P_{C 2}^{(i)}-\left\{\beta+\alpha_{3}\right\} P_{O}^{(i)}+\beta_{3} P_{C 5}^{(i)},
\end{aligned}
$$

For definition of receptor states and transition rates see Fig. 4.1. Each receptor has to be in one of its available states, so that

$$
P_{C 1}^{(i)}+P_{C 2}^{(i)}+P_{C 3}^{(i)}+P_{C 4}^{(i)}+P_{C 5}^{(i)}+P_{C 6}^{(i)}+P_{O}^{(i)}=1
$$

holds.

After specifying the initial conditions, here $P_{C 1}^{(i)}=1$ and $P^{(i)}=0$ for all other states - all receptors are initially in the closed unbound state - the set of Eqs. 4.10 is solved numerically for each receptor using a forth-order Runge Kutta method (Press et al., 1992). This yields for instance the open probability $P_{O}^{(i)}(t)$ of each of the $n_{\text {rec }}$ receptors, which can then be averaged to gain the total, averaged synaptic response of the receptor population $P_{O}^{(t o t)}(t)=\frac{1}{n_{\text {rec }}} \sum_{i=1}^{n_{\text {rec }}} P_{O}^{(i)}(t)$.

Because the number of receptors is small at central synapses, spatial fluctuations might not be negligible, giving rise to fluctuations in the EPSC's (see below). Modeling individual receptors in a local time-dependent concentration-field, we treat these fluctuations properly and are furthermore able to investigate the effects of different spatial arrangements of the receptors on the EPSC's. The only approximation in our model of chemical kinetic equations is to neglect variations in transmitter concentration due to the binding to and unbinding from postsynaptic receptors. This approximation will be tested by comparison of the results obtained from chemical kinetics to averages over many Monte Carlo runs in the following section. We expect that our assumption is justified for central synapses, where 1000-4000 transmitter molecules interact with 20-100 postsynaptic receptors.I All other theoretical models, which are used to calculate EPSCs by numerically solving the diffusion equation, employ the same approximation.

${ }^{2}$ The situation at the neuromuscular junction is quite different, where the high number of receptors may affect the transmitter concentration drastically. 


\subsection{Correspondence of kinetic and Monte Carlo model}

The basic assumption of our kinetic model is that fluctuations of neurotransmitter concentration due to binding and subsequent unbinding of transmitter molecules by postsynaptic receptors can be neglected. To test this assumption, we compare open probabilities $P_{O}^{(t o t)}$ as calculated from the kinetic model with averages over 500 Monte Carlo simulations, using the same amount of released transmitter, the same point of release and the same receptor population. Possible differences in the results are due to transmitter fluctuations because of binding and unbinding. As shown below in Fig. $4.5 B$ the average open probability generated by 500 Monte Carlo runs is in good correspondence with the result from the kinetic model for the same population of 30 receptors. Fluctuations due to binding and unbinding of transmitter by receptors can safely be neglected. As displayed in Fig. 4.4 there is only a small difference between the open probabilities due to these concentration fluctuations, - even if 300 receptors, which exceed the experimentally estimated number of glutamatergic postsynaptic receptors by far (Jonas et al., 1993; Spruston et al., 1995) compete for transmitter and contribute to transmitter depletion. We therefore conclude that the kinetic model is satisfactory for the calculation of averaged quantities and in the following we will use it to study systematic effects of different receptor distributions.

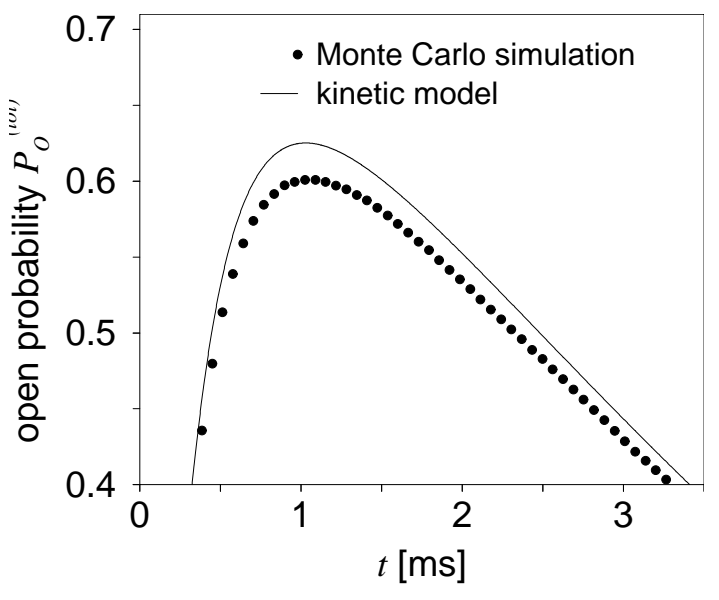

Figure 4.4.: Difference in the theoretical open probability $P_{O}^{(t o t)}$ between the kinetic model, where changes due to transmitter binding are neglected (solid line) and between the results of computer simulation, that include transmitter binding by 300 postsynaptic receptors and where $P_{O}^{(t o t)}$ has been averaged over 500 runs (black circles). Parameters given by the values in Tab. 4.3.

The agreement of the kinetic model and the Monte Carlo simulations shows that buffering of transmitter by binding to postsynaptic receptors is a small effect. Hence the amount of neurotransmitter released is not the limiting factor for maximal activation at central synapses. 

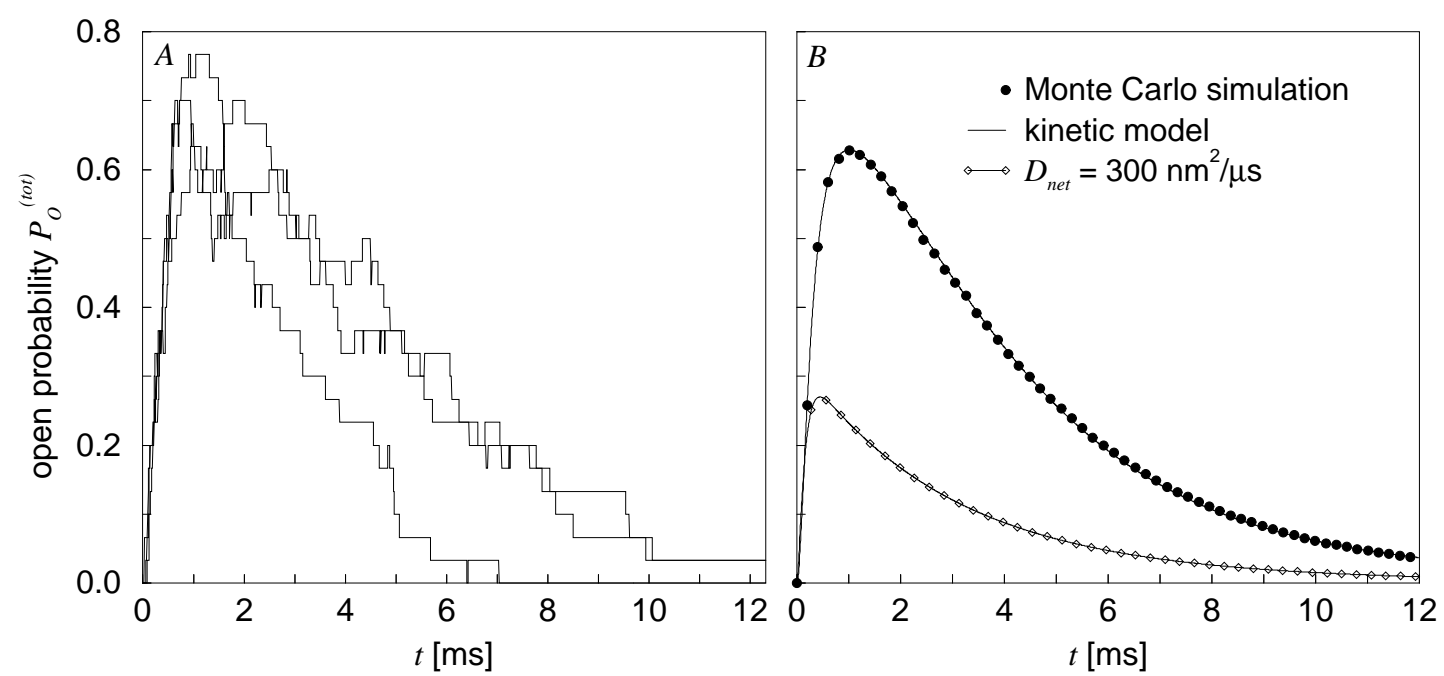

Figure 4.5.: Open probability $P_{O}^{(t o t)}$ as a function of time (parameters given by the values in Tab. 4.3). Three examples for the time course of $P_{O}^{(t o t)}$ resulting from a single simulation run. $B$ ) The simulated $P_{O}^{(t o t)}$ (black circles), averaged over 500 runs, is compared to results from local chemical kinetics (solid line). Also shown is $P_{O}^{(t o t)}$ as caused by a larger diffusion coefficient of $D_{\text {net }}=300 \mathrm{~nm}^{2} / \mu \mathrm{s}$ (white squares).

\subsection{Results for hippocampal synapses}

Miniature responses of a single synaptic bouton are modeled by computing the responses of a population of 30 receptors, randomly distributed over the PSD of Radius $R=200 \mathrm{~nm}$, exposed to 3000 transmitter molecules, which are released from a single vesicle in the center of the PSD. The small value of the diffusion coefficient $D_{\text {net }}$ which has been estimated in the previous chapter is used, and the total number of open channels, i.e., the open probability $P_{O}^{(t o t)}$ as a function of time is calculated. The signal of a single Monte Carlo run is quite noisy, as shown in Fig. 4.5A. To obtain a quantitative measure of the fluctuations we perform 500 runs and calculate the maximum amplitude, decay and rise time. A histogram of these values is presented in Fig. 4.6. The distribution of maximum amplitudes has a mean and standard deviation of 20.6 \pm 2.3 open channels, corresponding to an open probability of $(\sim 69 \%)$, as compared to the experimental value for unitary EPSCs in reduced extracellular $\mathrm{Ca}^{2+}$ of $\sim 72 \%$ (Jonas et al., 1993). For the distribution of rise times (defined as the time elapsed between $20 \%$ and $80 \%$ of the maximum) we find a mean and standard deviation of $0.51 \pm 0.26 \mathrm{~ms}$, compared to the experimental values $0.5 \pm 0.2 \mathrm{~ms}$ of Jonas et al. For the distribution of decay times we observe $4.05 \pm 1.15 \mathrm{~ms}$ compared to $4.1 \pm 0.9 \mathrm{~ms}$ of Jonas et al., 1993. The observed strong fluctuations are in good agreement with experiments and mainly due to the inherent noise in receptor kinetics. 

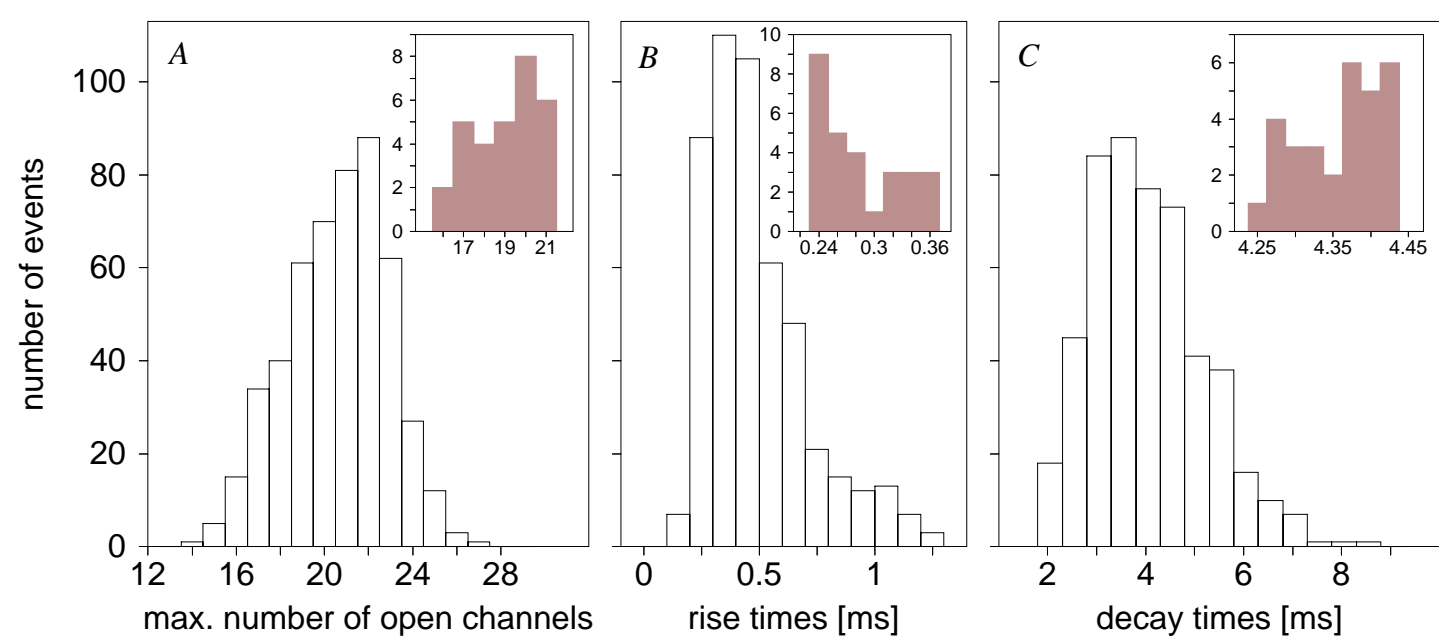

Figure 4.6.: Distribution of $A$ ) maximum amplitudes, $B$ ) rise times and $C$ ) decay times for 30 randomly distributed receptors on a PSD of $R=200 \mathrm{~nm}$, exposed to 3000 transmitter molecules released from a single vesicle in the middle of the synaptic disc $\left(r_{\mathrm{abs}}=500 \mathrm{~nm}, D_{\text {net }}=30 \mathrm{~nm}^{2} / \mu \mathrm{s}\right.$, rest of parameters from Tab. 4.3). The gray columns in the insets indicate the range of variation due to the spatial distribution of the receptors, calculated from the kinetic model for each receptor position.

Also displayed is the open probability for a diffusion coefficient which is 10 times larger and often used for theoretical models of transmitter diffusion, (Busch and Sakmann, 1990; Holmes, 1995; Uteshev and Pennefather, 1997; Wahl et al., 1996). It is obvious from Fig. $4.5 B$ that the experimentally determined open probabilities of approximately $60-70 \%$ for AMPA/kainate receptors (Häusser and Roth, 1997; Jonas et al., 1993; Spruston et al., 1995) cannot be reproduced by the commonly used larger value of the diffusion constant $D_{\text {net }}$.

There are several sources of noise, in particular inherent noise in the receptor dynamics and fluctuations due to a spatial distribution of receptors. The Monte Carlo simulation includes both, whereas the noisy receptor dynamics has been averaged out in the kinetic model. This allows us to discriminate between the two noise sources. Within the kinetic model we calculate the open probability for receptors located at a given distance from the site of release, so that we know maximum amplitudes, rise and decay times as a function of distance between receptor and release site. For a given realization of the distribution we can draw a histogram of maximum amplitudes, rise and decay times, as shown in the inset of Fig. 4.6 (gray columns). Obviously the inherent noise of receptor kinetics is much stronger than the fluctuations due to random distances between receptors and release site. 

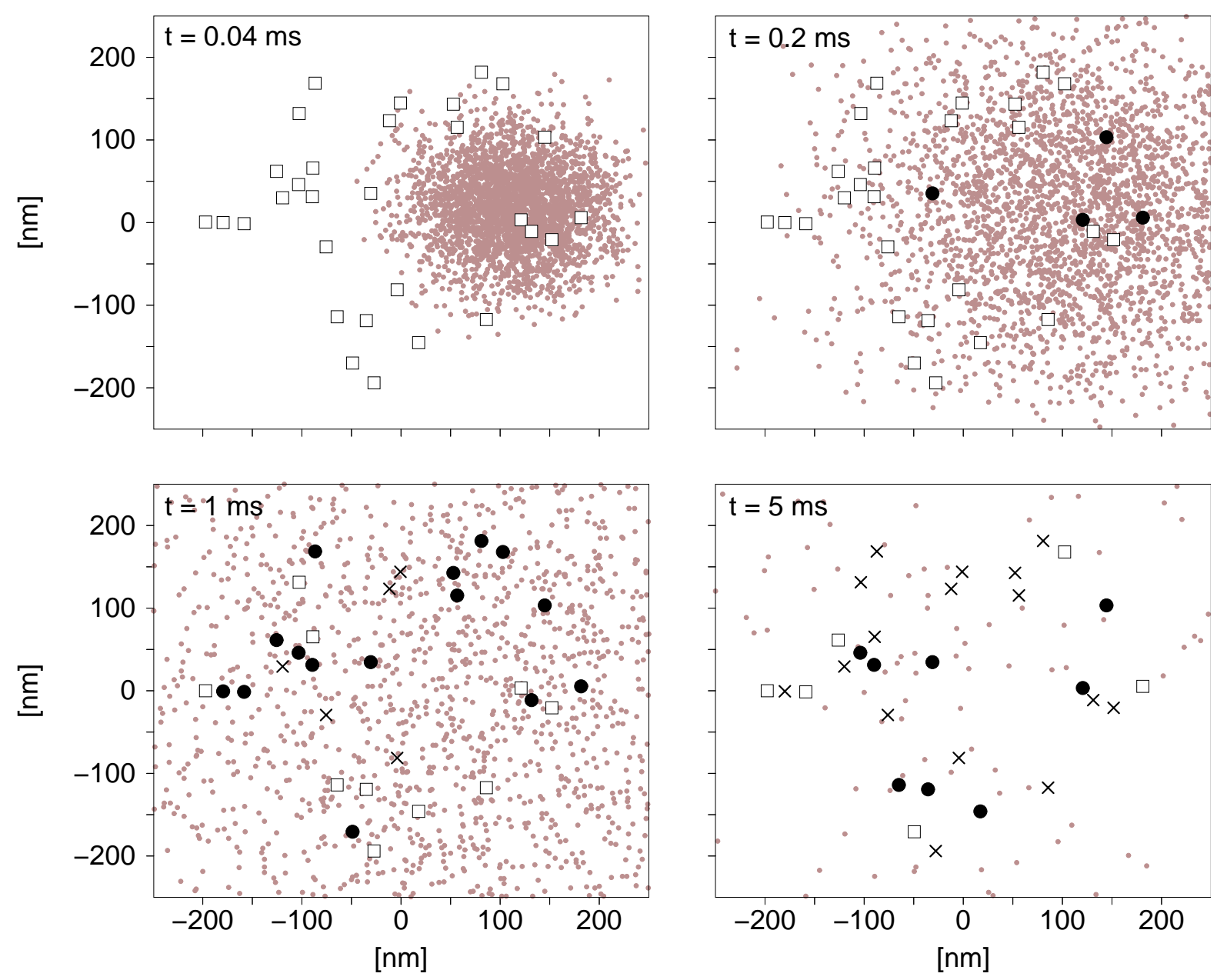

Figure 4.7.: Receptor distribution on a simple synapse; states of 30 randomly distributed receptors at times $t=0.04,0.2,1$, and $5 \mathrm{~ms}$ after release of a single vesicle (at a randomly chosen release site within the PSD), which contains 3000 molecules (small grey circles); rest of parameters as in Fig. 4.6. At time $t=0$ all the receptors are in the closed unbound state $C 0$ (white squares, inactive states $C 1$ and $C 2$ also displayed as white squares), but start to open (state $O$, black circles) or desensitize (inactive states $C 3, C 4$, and $C 5$, black crosses).

\section{Effects of different receptor arrangements}

Our model is used to study the effect of different spatial arrangements of receptors on the postsynaptic side. Changes of the synaptic geometry from simple to perforated (clustered) synapses have been suggested as a possible mechanism of long-term changes in synaptic efficacy (= long-term potentiation, LTP) (Edwards, 1995b). We compare EPSCs of a simple synapse with an unperforated PSD (Fig. 4.7) with a perforated synapse as shown in Fig. 4.8. Each cluster of the perforated PSD is associated with a possible release site 


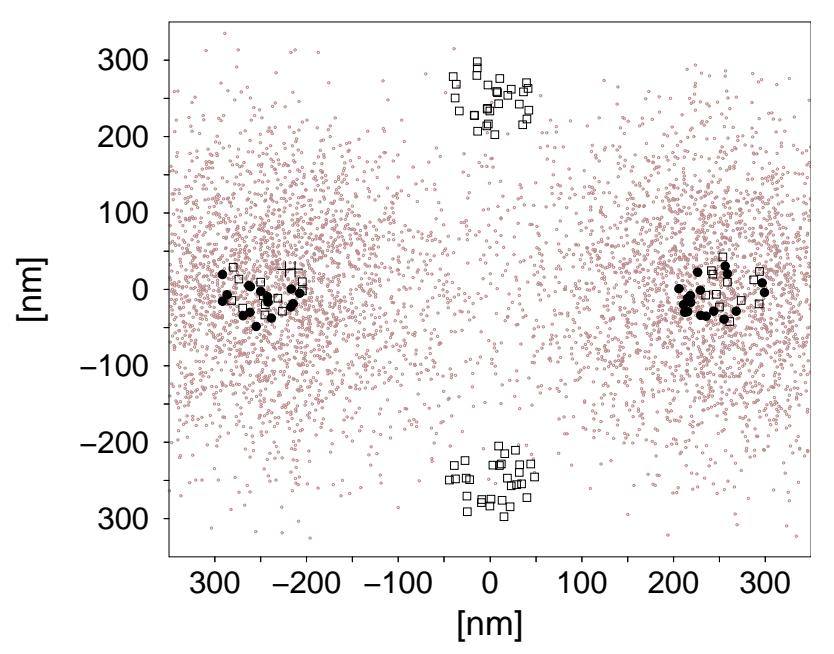

Figure 4.8.: States of 120 receptors distributed in four clusters, each with a diameter of $100 \mathrm{~nm}$ at times $t=0.2 \mathrm{~ms}$. Two vesicles have been released at time $t=0$ in the center of two clusters, each vesicle containing 3000 molecules; rest of parameters as in Fig. 4.7.

of presynaptic vesicles and contains the same number of receptors as distributed across the simple PSD (30 receptors).

First, the simulation is used to visualize the effects of transmitter diffusion on the receptor dynamics. Four snapshots of 30 randomly distributed receptors on a PSD of $200 \mathrm{~nm}$ radius at different times after the release of a single vesicle are shown in Fig. 4.7. One observes how the opening of channels coincides with the spreading of transmitter. Following the release of a single vesicle containing 3000 molecules $60 \%$ of the receptors are in one of the inactive states $C 3, C 4$, or $C 5$ after the transmitter has been cleared from the cleft. Figure $4.9 \mathrm{~A}$ indicates that the simple synapse is saturated to $\sim 70 \%$ by the release of a single vesicle and saturates after the simultaneous release of two vesicles (as discussed in e.g., Busch and Sakmann, 1990, Edwards, 1991, Bennett et al., 1997), while in contrast the amplitude distribution at perforated synapses (Fig. 4.9B $B$ ) doesn't exhibit saturation after the release of two vesicles. The distribution of rise times becomes narrower and shifts towards faster rise times (Fig. 4.10) as more vesicles are released. At the perforated PSD the change in the distribution of rise times (Fig. 4.10 $B$ ) is stronger than for the simple synapse (Fig. 4.10 A) because the broadening of the rise time distribution for the release of one vesicle is caused by the late activation of receptors from distant clusters. Distributions of decay times do not change systematically with the amount and position of transmitter released (data not shown) and are mostly determined by the stochasticity of the individual receptor dynamics. 

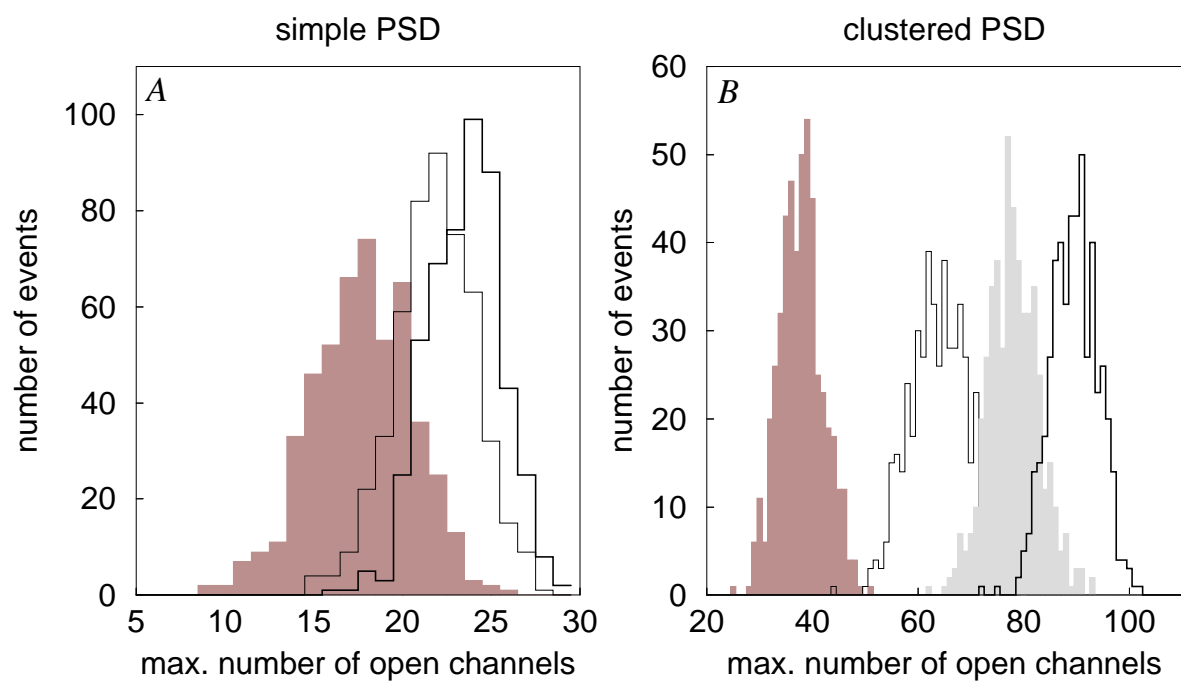

Figure 4.9.: Effect of multiple release on the distribution of maximum amplitudes of A) 30 receptors distributed randomly across a simple PSD (Fig. 4.7) and B) 120 receptors distributed in four receptor clusters of 30 receptors each (Fig. 4.8). Amplitude distribution for one (dark gray columns), two (solid line), three (light grey columns) and four (thick solid line) vesicles released, generated from 500 simulation runs (parameters as in Fig. 4.7 and 4.8).
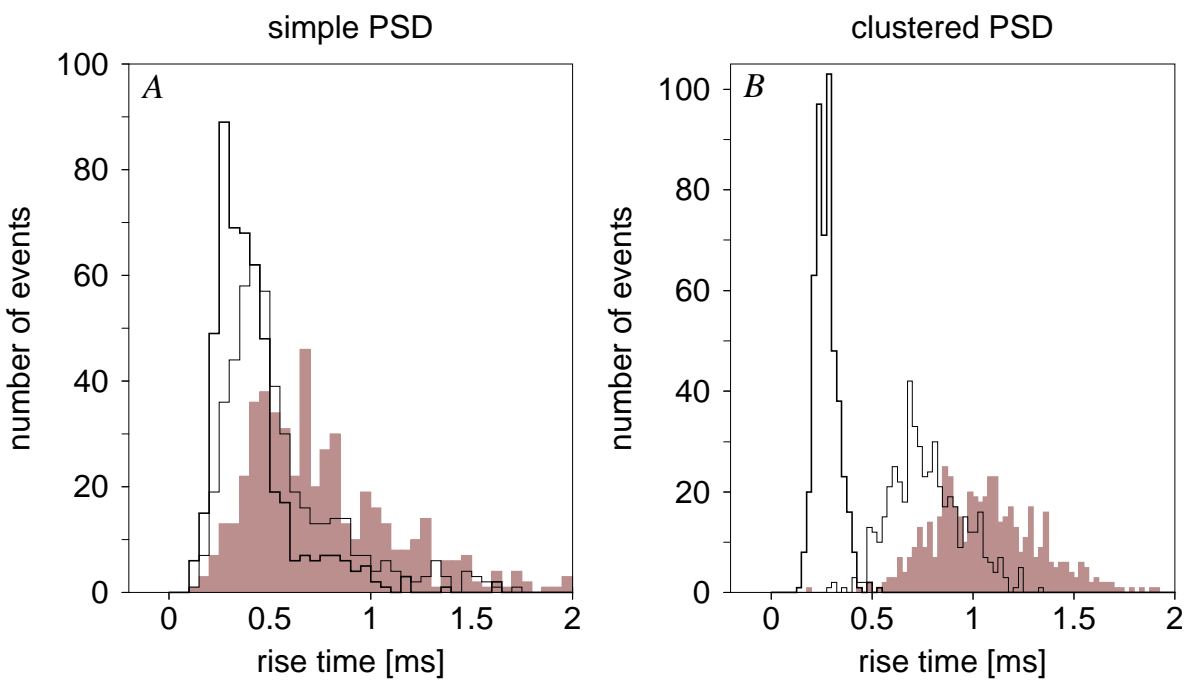

Figure 4.10.: Effect of multiple release on the distribution of rise times of $A$ ) 30 receptors distributed randomly across a simple PSD (Fig. 4.7) and B) 120 receptors distributed in four receptor clusters of 30 receptors each (Fig. 4.8). Distribution of rise times for one (dark gray columns), two (solid line) and four (thick solid line) vesicles released, generated from 500 simulation runs (parameters as in Fig. 4.7 and 4.8). 


\section{Postsynaptic mechanisms for an increase in synaptic efficacy}

As seen in the previous paragraph the PSD is partially saturated by one vesicle and only a gradual increase in postsynaptic current is observed, if more than one vesicle per bouton is released. This suggests a postsynaptic mechanism for potentiation. [3 In the case of strong saturation, changes in vesicle content or changes in the incidence of multi-vesicular release will hardly change the postsynaptic current and hence cannot serve as mechanisms for potentiation.
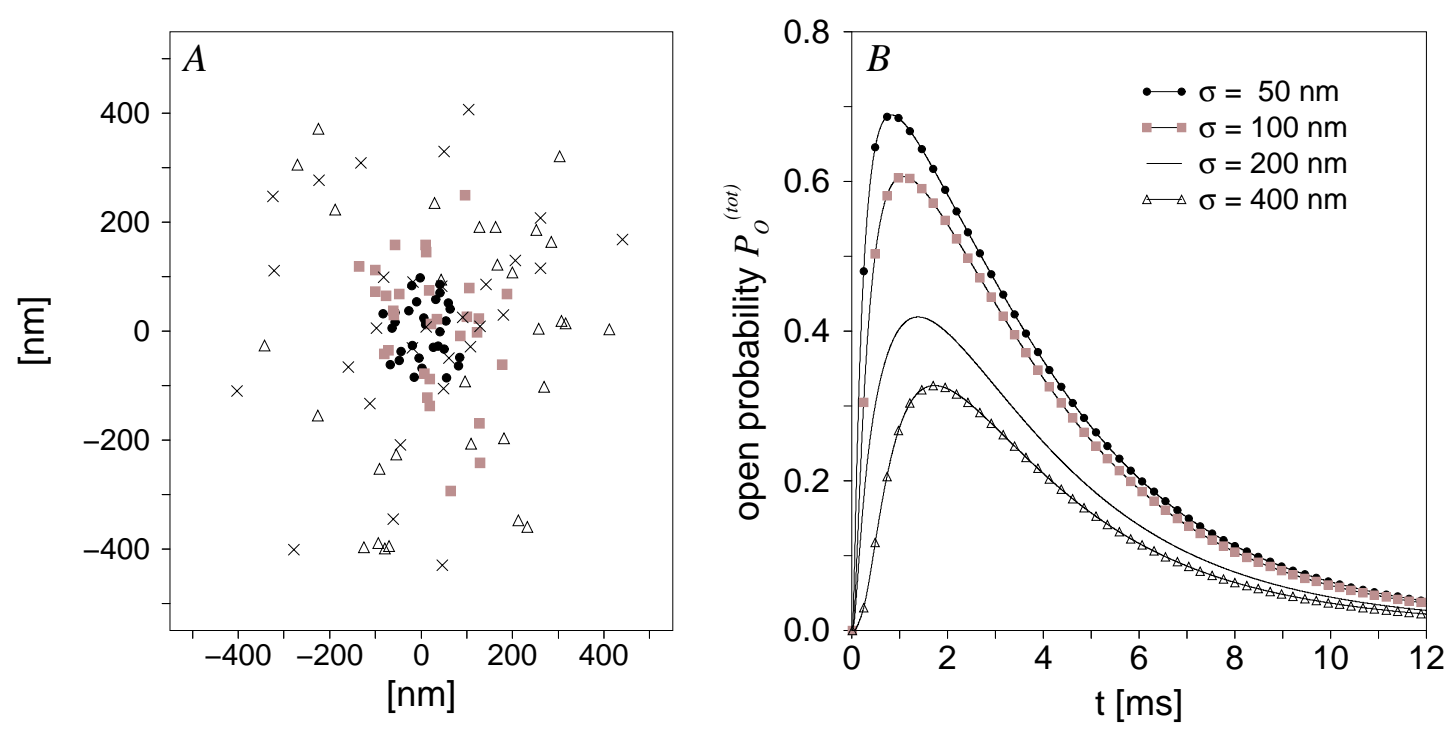

Figure 4.11.: A) 30 receptors distributed according to a Gaussian distribution centered around the point of release, which here is chosen in the middle of synaptic disc. Here $\sigma$ denotes the standard deviation of the Gaussian. B) Total open probability $P_{O}^{(t o t)}$ as function of time for the receptor distributions in $A$, as calculated from the kinetic model; rest of parameters as in Fig. 4.6, $\sigma=50$ (black circles), 100 (gray squares), 200 (black crosses, solid line) and $400 \mathrm{~nm}$ (white triangles).

In the following we discuss changes in synaptic efficacy due to synaptic modifications on the postsynaptic side. We first study rather smooth changes in the distribution of receptors, e.g., the size or the shape of the PSD is varied, while the total number of receptors is kept constant, as suggested as a first step of change in synaptic structure by Edwards (1995a). A possible arrangement of receptors on the postsynaptic membrane located near the point of transmitter release (here in the middle of the synaptic disc) is shown in Fig. 4.11 A. The same number of receptors have been distributed according to a Gaussian distribution, where the standard deviation $\sigma$ characterizes the average distance of the receptors from the point of release. For smaller values of $\sigma$ the receptors

\footnotetext{
${ }^{3}$ We do not discuss here an overall increase in release probability, resulting in an activation of silent synaptic boutons.
} 
are clustered closer to the point of release, while for higher values of $\sigma$ the receptor distribution resembles a random homogeneous receptor distribution. The calculated open probability $P_{O}^{(t o t)}$ in Fig. $4.11 B$ reveals that a clustering of postsynaptic receptors causes a relatively weak potentiation of the peak amplitude, e.g., a 15 to $20 \%$ change for $\sigma=50$ and $100 \mathrm{~nm}$. This is to be expected, because of the abundance of neurotransmitter molecules and the fact that the transmitter concentration equilibrates within a few hundred $\mu \mathrm{s}$ (small inset in Fig. 3.2), which is fast compared to the receptor kinetics.7 To double the open probability, keeping the total number of receptors fixed, requires a 64 times smaller active zone. Such extreme changes seem to be more realistic in the context of structural changes of synaptic morphology.

A much more effective potentiation is achieved by increasing the number of postsynaptic receptors. We find that the maximum amplitudes of EPSCs are directly proportional to the total number of receptors (Fig. 4.12 $A$ ). The normalized open probability for the same PSD with 30, 80 and 150 receptors is compared in Fig. 4.12B. The differences between the three curves are very small approximation linearly with the total number of receptors.
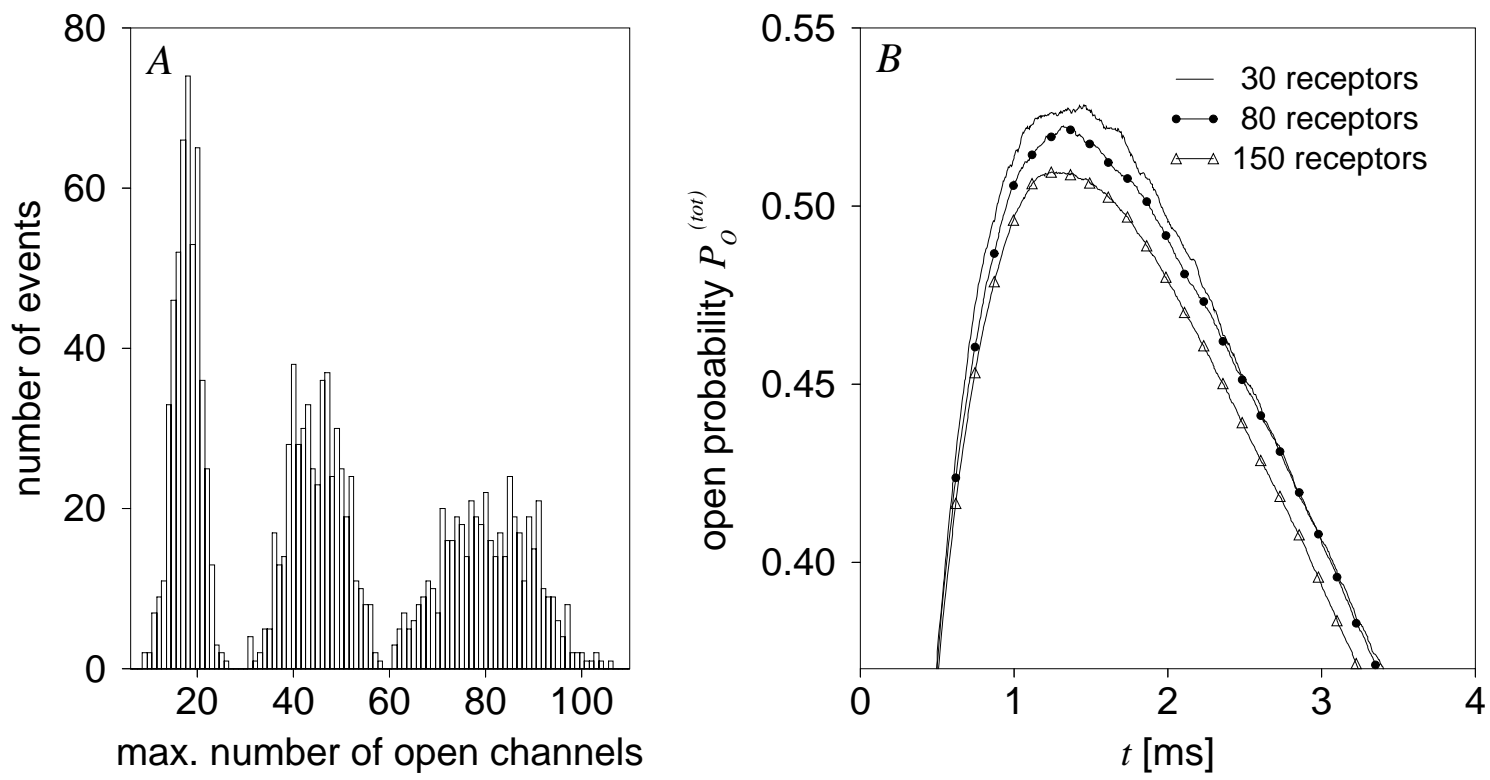

Figure 4.12.: A) Distributions of amplitudes for 30, 80 and 150 receptors distributed randomly on a PSD of fixed size $(R=200 \mathrm{~nm})$ for a random point of release, generated from 500 runs, rest of parameters as in Fig. 4.6 and Tab. 4.3. $B$ ) Corresponding open probabilities $P_{O}^{(t o t)}$, averaged over 500 runs, for 30 (solid line), 80 (black circles) and 150 receptors (white triangles).

\footnotetext{
${ }^{4} \mathrm{~A}$ larger diffusion coefficient speeds up the equilibration of transmitter across the PSD and further reduces the influence of spatially different receptor arrangements.

${ }^{5}$ This result again confirms our approach that the transmitter depletion due to binding to postsynaptic receptors is a small effect; see also Fig. 4.4
} 
The number of receptors can be increased in at least two qualitatively different ways: Either the geometry of the synapse is left unchanged or alternatively new boutons are created. [ Edwards (1995a) has suggested perforation of a simple synapse into several clusters of receptors, where the different receptor clusters act as nearly independent release sites, together with an overall increase in the number of receptors as an effective mechanism for potentiation. Following this idea multiple release at perforated synapses could account for a skew in amplitude distributions of miniature currents (for a detailed discussion see Edwards, 1995b). We follow this suggestion and assume four possible release sites: at the simple synapse they are randomly distributed across the PSD as in Fig. 4.7, while at the perforated synapse each release site is associated with a receptor cluster (Fig. 4.8). The probability $p$ for release of a vesicle is assumed to be the same for all four release sites. As displayed in Fig. 4.13 the shape of the amplitude distribution varies with a change in the release probability $p$ from 0.05 to 0.4 from a non-skewed to a skewed distribution for the perforated synapse, while the distribution for the simple synapse doesn't seem to change systematically. For higher release probabilities the skew again vanishes or even appears towards smaller amplitudes. These changes in miniature amplitude distributions should occur with systematic alterations in release probability, e.g. by increase of intra- and extracellular calcium or change in temperature. They are currently point of further experimental investigation and first results have been presented in preliminary form (Trommershäuser et al., 1997).

${ }^{6}$ Another possibility may be the activation of silent synapses. 

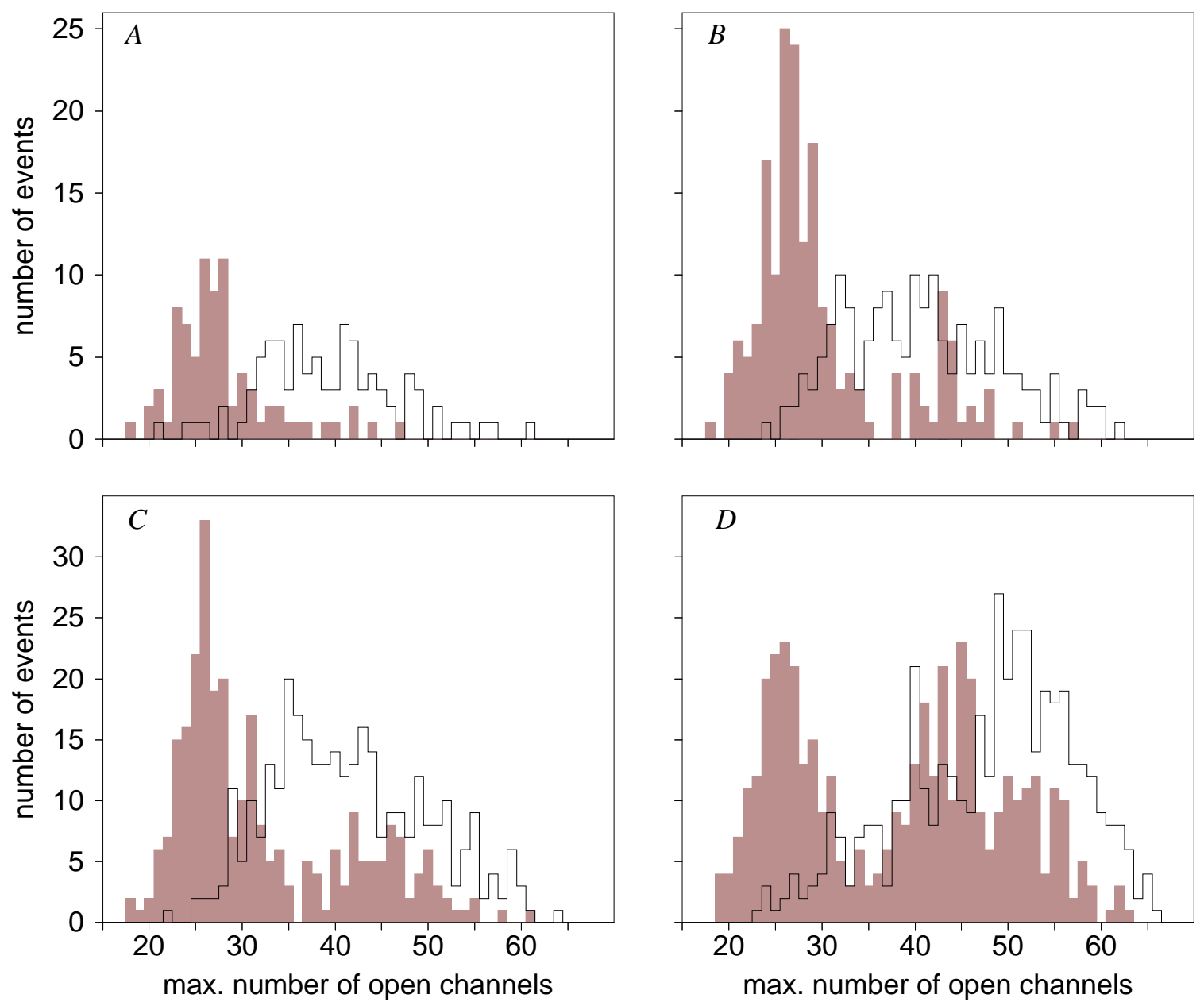

Figure 4.13.: Distributions of peak amplitudes (max. number of open channels), each distribution generated for 120 receptors from 500 runs. Different panels show distributions for varying release probabilities: $A) \mathrm{p}=0.05 /$ failure rate $\sim 80 \%, B) \mathrm{p}=0.1 /$ failure rate $\sim 66 \%, C) \mathrm{p}=0.2 /$ failure rate $\sim 41 \%$ and $D$ ) $\mathrm{p}=0.4$ / failure rate $\sim 12 \%$ The gray distributions are from perforated synapses (parameters as in Fig. 4.8). The corresponding white distributions result from the release of four vesicles at random release sites across the PSD at a simple synapse with 120 receptors (rest of parameters as in Fig. 4.6). The failure rates denote the percentage of events without the release of any vesicle. 


\subsection{Results for brainstem interneurons}

In this section we study AMPA-receptor mediated miniature currents recorded from interneurons in the brainstem, not only to analyze neurons located in a different area of the brain, but also because the recorded postsynaptic currents reveal unusually large fluctuations in amplitudes and decay times as displayed in Fig. 4.14. In the following we will use Monte Carlo simulations to study the source of the observed variability of amplitudes, rise and decay times.
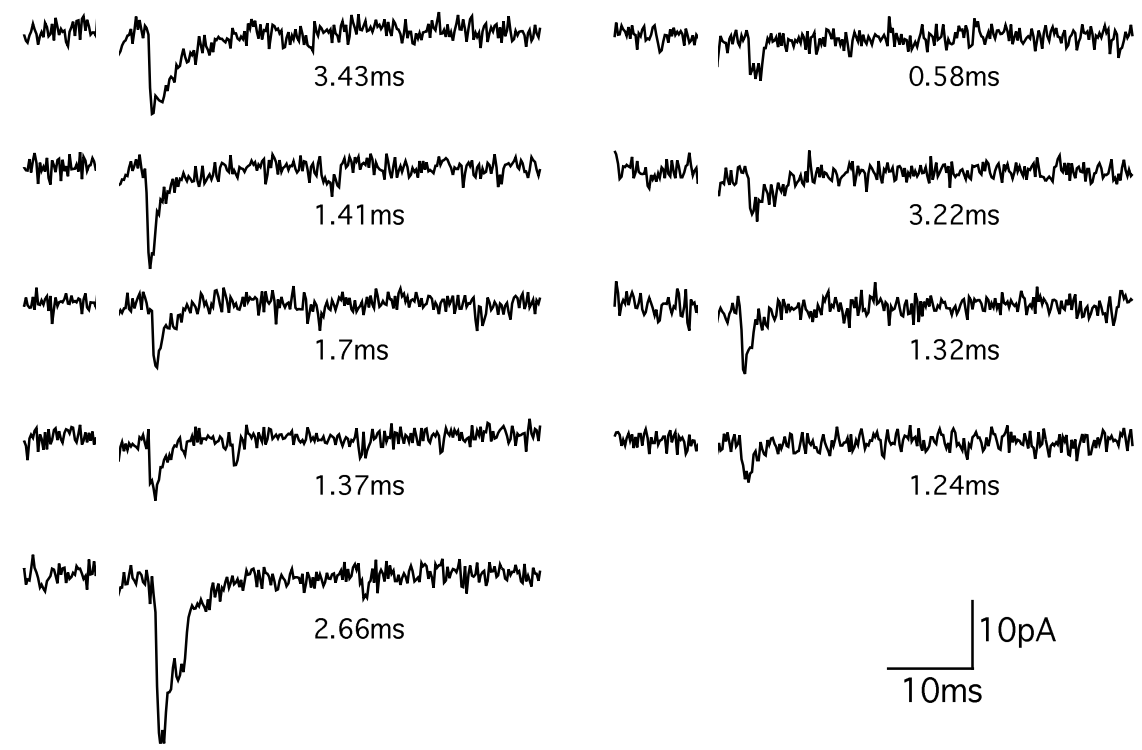

Figure 4.14.: Fluctuations in the time course of EPSCs recorded during single fiber stimulation in the presence of low extracellular calcium concentration $(0.8 \mathrm{mM})$ at a failure rate of $58 \%$ (taken from Titz \& Keller, 1996).

Several mechanisms have been suggested as source of synaptic variability and will be discussed in the following: Most likely miniature currents are recorded from various synaptic boutons, which might differ regarding the postsynaptic receptor number (Borst et al., 1994; Edwards et al., 1990; Nusser et al., 1997) and receptor distribution (Wahl et al., 1996), vesicle content (Liu et al., 1999) and synaptic morphology (Edwards, 1995a; Walmsley et al., 1998). The latter, as well as heterogeneities in the transmitter-transporter interaction (Rusakov and Kullmann, 1998; Trommershäuser et al., 1999) have been suggested to affect the transmitter dynamics in- and outside the cleft (Kullmann et al., 1999). Furthermore it has been pointed out (Bier et al., 1996; Faber et al., 1992; Trommershäuser et al., 1999) that due to a small number of postsynaptic receptors, the intrinsic noise of the receptor dynamics should provide a significant contribution to synaptic variability.

The time course of mEPSCs exhibits large variations as shown in Fig. 4.15. Recordings of miniature EPSCs yield distributions of maximum amplitudes (52.8 \pm 26.4 channels 
open at peak amplitude, $\mathrm{CV}=0.5)$, rise $(0.30 \pm 0.11 \mathrm{~ms}, \mathrm{CV}=0.37)$ and decay times $(2.64 \pm 1.30 \mathrm{~ms}, \mathrm{CV}=0.49)$ as displayed in Fig. 4.15. These fluctuations in the decay times are larger than those reported for AMPA mediated mEPSCs at CA3 and CA1 regions of rat hippocampus (Jonas et al., 1993: CV =0.22; Atassi \& Glavinovic, 1999: $\mathrm{CV}=0.34)$ and computed in Sec. 4.5.

Notice, that rise times are still relatively homogeneous indicating that filtering effects due to the cable properties of the dendritic tree are small. [ Large variations are only observed in EPSC amplitude and decay time constants. Rise times, amplitudes or decay times do not correlate significantly (Fig. 4.16). These findings allow the conclusion that filtering effects contribute weakly to the observed variability. Hence we will not take dendritic filtering into account in our theoretical analysis. To identify the underlying elementary events, a detailed electrophysiological analysis of miniature EPSCs in NTS interneurons has been performed and the results are in the following compared with the results of Monte Carlo simulations.

Information about the synaptic geometry and the number of transmitter molecules released is taken from Edwards (1995) and Bruns \& Jahr (1995). The distribution of rise times is used to yield an estimate of the effective diffusion constant $D_{\text {net }}=40 \mathrm{~nm}^{2} / \mu \mathrm{s}$, which is in accordance with the range calculated in Sec. 3.3. The set of parameters used to simulate mEPSCs at brainstem interneurons is summarized in Tab. 4.4.

Table 4.4.: Parameters as used in the Monte Carlo simulations of single synaptic boutons of brainstem interneurons (rest of parameters as noted in Tab. 4.3).

\begin{tabular}{lc}
\hline Geometry: & \\
receptor density & $1600 \pm 800$ receptors $/ \mu \mathrm{m}^{2}$ \\
postsynaptic density (PSD), radius $\mathrm{r}_{\mathrm{PSD}}$ & $\sim 150 \mathrm{~nm}$ \\
absorbing boundary, radius $\mathrm{r}_{\text {abs }}$ & $\sim 500 \mathrm{~nm}$ \\
& \\
Transmitter: & 4000 \\
no. of molecules/vesicle, $\mathrm{N}_{\mathrm{T}}$ & $40 \mathrm{~nm}^{2} / \mu \mathrm{s}$ \\
diffusion constant, $D_{\text {net }}$ &
\end{tabular}

\footnotetext{
${ }^{7}$ If the recorded responses were caused by a wide spread of synaptic contacts across the dendritic tree, a heterogeneity in decay times could be caused by filtering of the synaptic responses due to the cable properties of the dendritic tree. Nevertheless, filtering would not only appear in the modification of decay times, but also effect the rise times, causing a broad spectrum of rise times and correlations between prolonged rise and decay times (Rall and Agmon-Snir, 1998).
} 

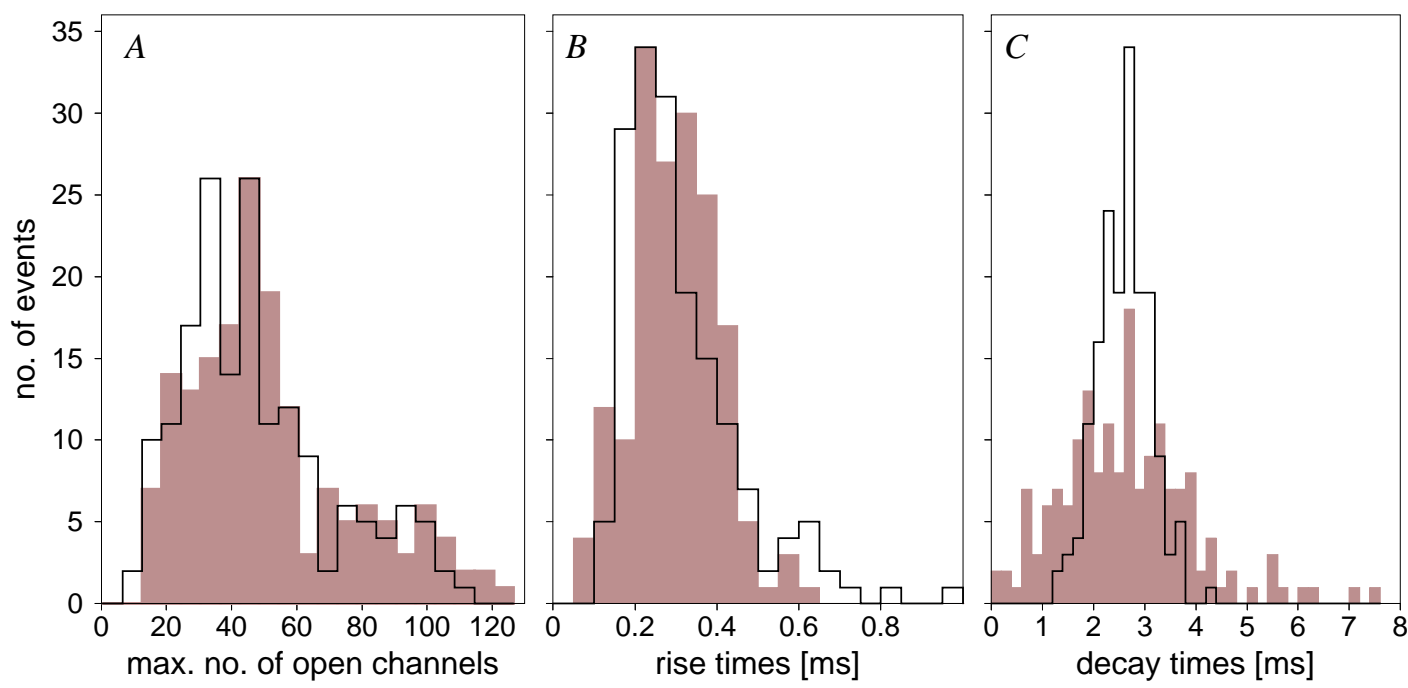

Figure 4.15.: Distributions of miniature amplitudes $(A)$, rise $(B)$ and decay times $(C)$ as recorded in low extracellular $\mathrm{Ca}^{2+}$ (gray columns) and as generated from Monte Carlo simulations (white columns). Simulations yield a maximum of open channels of $47.8 \pm 23.2$ channels (experiments:52.8 \pm 26.4 channels), rise times of $0.32 \pm 0.18 \mathrm{~ms}$ (experiments: $0.30 \pm 0.11 \mathrm{~ms}$ ) and decay times of $2.61 \pm 0.48 \mathrm{~ms}$ (experiments: $2.64 \pm 1.30 \mathrm{~ms}$ ). Monte Carlo simulations were run using the parameters in Tab. 4.4 and 4000 transmitter molecules for the content of a single vesicle and released from a release-site, which for every run was chosen randomly within the PSD of radius $\mathrm{r}_{\mathrm{PSD}}=150 \mathrm{~nm}$.

\section{Amplitude distribution of miniature EPSCs}

In the simulations it has been assumed that a single mEPSC is caused by the release of 4000 glutamate molecules from a single vesicle at a randomly chosen release site located within a PSD of $300 \mathrm{~nm}$ diameter. The remaining geometric parameters are given in Tab. 4.4. The chosen synaptic scenario implies a residence time of glutamate in the cleft of $\langle T\rangle=0.43 \pm 0.04 \mathrm{~ms}$ as calculated by Eq. 3.4.

Monte Carlo simulations have been performed to study the source of variation observed in the time course of miniature amplitudes. Monte Carlo simulations of a single synaptic bouton with a fixed number of 85 postsynaptic receptors yield a non-skewed distribution of amplitudes ( $53.1 \pm 4.4$ channels open at peak amplitude), which is too narrow to account for the range of experimentally observed fluctuations in the distribution of miniature amplitudes (52.8 \pm 26.4 channels) as displayed in Fig. 4.17. The simulation results indicate that following the release of a single vesicle about $63 \pm 5 \%$ of all channels open. Hence, as expected from the results of outside-out patch-experiments (see Sec. 4.1.3) and our Monte Carlos simulation at hippocampal synapses (Sec. 4.5) the population of postsynaptic receptors is saturated to a large extent by the release of a single vesicle. Consistently variations in vesicle content fail to cover the range of miniature am- 

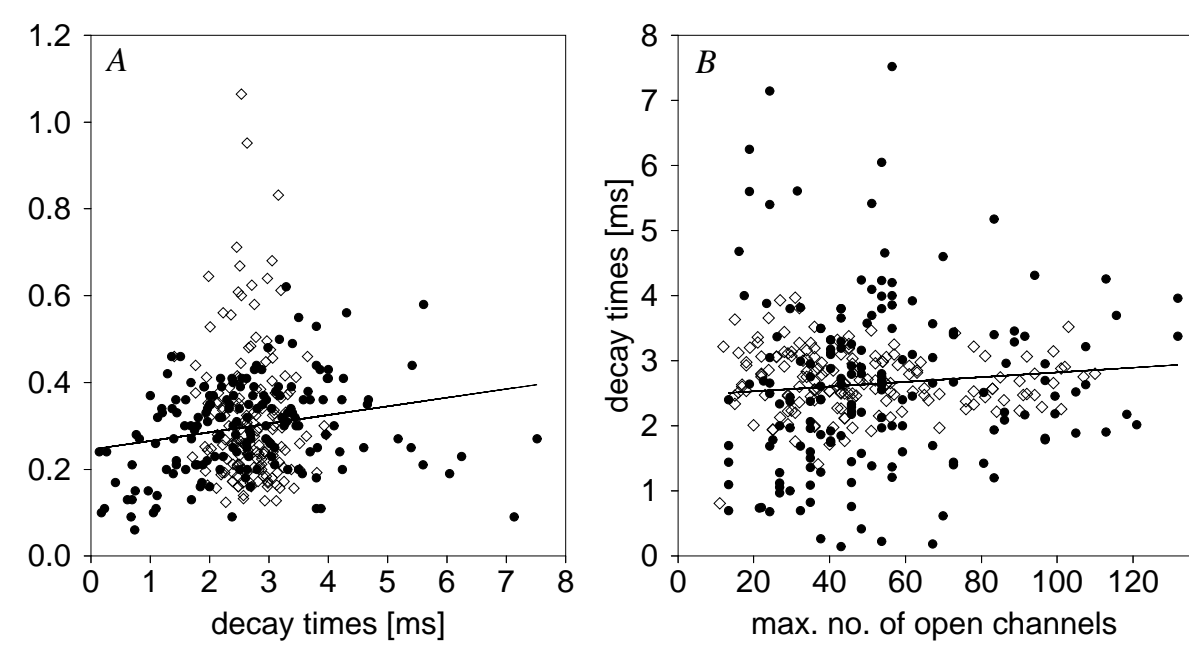

Figure 4.16.: Rise times as function of decay times $(A)$ and decay times as function of maximum amplitudes $(B)$ amplitude. Black circles indicate the results from recordings of mEPSCs, white diamonds the results from Monte Carlo simulations (see Fig. 4.15 and Tab. 4.4 for parameters). The black line indicates the correlation of rise and decay times $(A: \mathrm{r}=0.16)$, and of decay times and amplitudes $(B: \mathrm{r}=0.07)$.

plitudes, if the number of postsynaptic receptors is fixed: As shown in Fig. 4.17 a strongly fluctuating vesicle content (4000 \pm 2000 molecules randomly assigned as vesicle-content) does not significantly increase the spectrum of miniature amplitudes. While vesicles containing less than 1200 molecules cause smaller maximum amplitudes, the complementary effect for vesicles with a large amount of transmitter molecules does not occur due to the saturation of postsynaptic receptors. Hence variations in the miniature amplitude distribution of brainstem interneurons are not explained by variations in vesicle content, i.e. are not located on the presynaptic side, but are due to fluctuations in postsynaptic receptor number.

The variation in maximum amplitudes is then caused by fluctuating currents from several synaptic boutons containing a variable number of receptors. We estimate this receptor number by assuming that about $70 \%$ of postsynaptic receptors are activated by the release of a single vesicle (Sec. 4.1.3). For every simulation run the number of postsynaptic receptors is calculated directly from our experiments by dividing the recorded miniature amplitudes by 0.7 and the single channel current of $0.372 \mathrm{pA}$ (Titz and Keller, 1997). This yields a receptor number of $77 \pm 39$ postsynaptic receptors in a synapse (corresponding to $47.8 \pm 23.2$ channels open at peak amplitude) and allows to correctly cover the range of variation observed in experimental mEPSCs (Fig. 4.15A). 


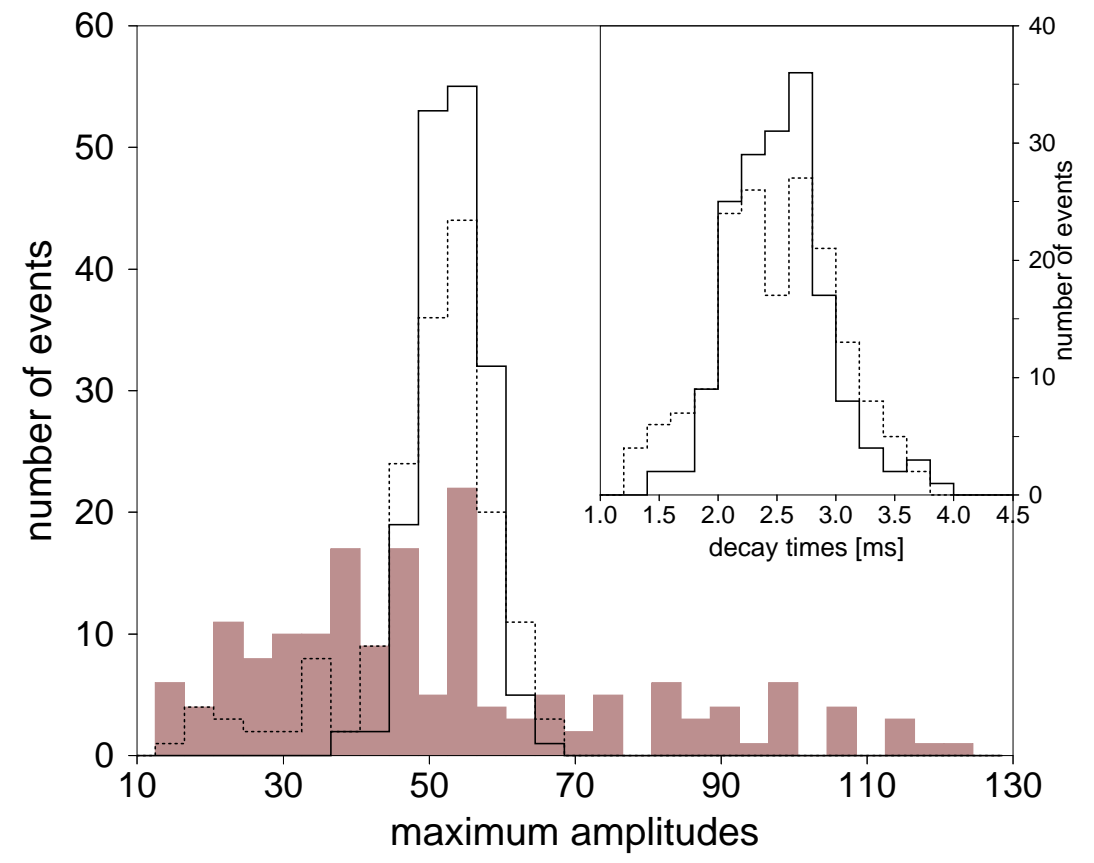

Figure 4.17.: Effect of fluctuations in vesicle content on amplitudes and decay times: Distributions of maximum amplitudes and decay times (small inset) as generated from Monte Carlo simulations for a fixed number of 85 postsynaptic receptors and the release of 4000 transmitter molecules (white columns, solid line: $53.1 \pm 4.4$ channels open at peak amplitude, decay times: $2.53 \pm 0.42 \mathrm{~ms}$ ) or a vesicle content, randomly chosen from a Gaussian distribution with a mean of 4000 molecules and a SD of 2000 molecules (white columns, dotted line, $49.5 \pm 10$ open channels; decay times: $2.5 \pm 0.52 \mathrm{~ms}$ ). Gray columns indicate the experimentally recorded miniature amplitudes.

\section{Distribution of rise times}

For several types of central synapses a large variety of PSD shapes has been observed (see Edwards, 1995 and Walmsley et al., 1998 for further references) and it has been suggested that variations in receptor distribution might contribute to synaptic variability of mEPSC amplitudes (Lim et al., 1999; Oleskevich et al., 1999; Wahl et al., 1996). Our results on hippocampal synapses however do not support this idea (Sec. 4.5): We have shown for synapses, which are saturated to a large extend by the release of a single vesicle, that due to the rapid equilibration of neurotransmitter across the synaptic cleft heterogenous receptor distributions do not cause a noticeable effect on amplitudes or decay times. If synaptic currents are expected to exhibit an effect due to the heterogeneity of receptor distributions it should occur in the distribution of rise times because of the late activation of receptors at larger distances from the point of release. The results displayed in Fig. 4.18 support this finding. Extremely large extensions of the PSD (diameter of 


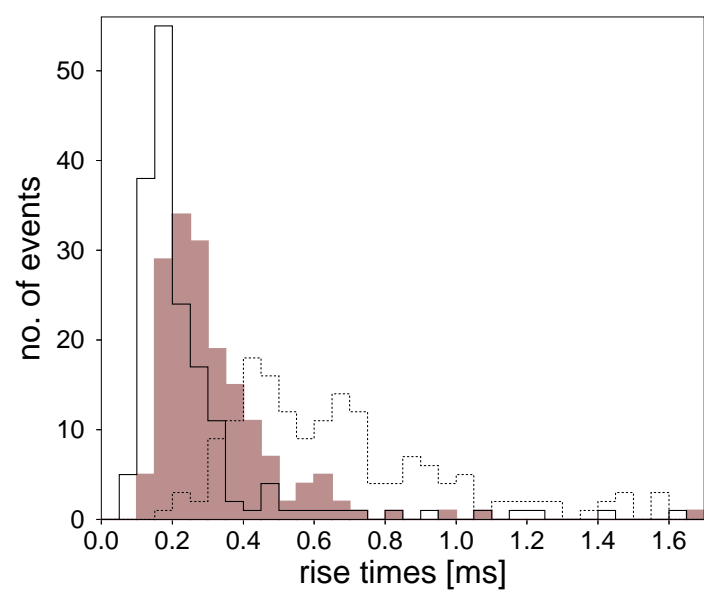

Figure 4.18.: Distribution of rise times for different extensions of the PSD; gray columns: $r_{\mathrm{PSD}}=75 \mathrm{~nm}$, rise times: $0.25 \pm 0.12 \mathrm{~ms}$; white columns/solid line: $r_{\mathrm{PSD}}=150 \mathrm{~nm}$, rise times: $0.32 \pm 0.18 \mathrm{~ms}$; white columns/dotted line: $r_{\mathrm{PSD}}=300 \mathrm{~nm}$, rise times: $0.70 \pm 0.37 \mathrm{~ms}$; rest of parameters as in Fig. 4.15 and Tab. 4.4.

$600 \mathrm{~nm}$ compared to $150 \mathrm{~nm}$ or $300 \mathrm{~nm}$ ) broaden the distribution of rise times (Fig. 4.18). Therefore a large variability in PSD shapes would create a broad distribution of rise times, which is not observed experimentally. We conclude that at brainstem interneurons postsynaptic receptors are distributed on average at about the same distance from the point of release on small PSDs with about $300 \mathrm{~nm}$ of diameter.

It should be mentioned in this context, that fluctuations in the receptor distribution only slightly modulate the distribution of amplitudes $\left(r_{\mathrm{PSD}}=75 \mathrm{~nm}\right.$ : $51.5 \pm 24.8$ channels; $r_{\mathrm{PSD}}=150 \mathrm{~nm}: 47.8 \pm 23.2$ channels; $r_{\mathrm{PSD}}=300 \mathrm{~nm}: 36.1 \pm 17.6$ channels $)$, do not systematically alter the distribution of decay times $\left(r_{\mathrm{PSD}}=75 \mathrm{~nm}: 2.6 \pm 0.5 \mathrm{~ms}\right.$; $\left.r_{\mathrm{PSD}}=150 \mathrm{~nm}: 2.61 \pm 0.48 \mathrm{~ms} ; r_{\mathrm{PSD}}=300 \mathrm{~nm}: 2.16 \pm 0.54 \mathrm{~ms}\right)$ and hence fail to account for the experimentally observed range of variation of amplitudes and decay times. Furthermore we find that the distribution of rise times is left unchanged for fluctuating vesicle content $(0.3 \pm 0.14 \mathrm{~ms}$, see also Fig. 4.17), variable receptor number $(0.32 \pm 0.18 \mathrm{~ms}$, Fig. $4.15 B)$ as compared to fixed number and fixed vesicle content $(0.27 \pm 0.1 \mathrm{~ms}$, Fig. 4.17). 


\section{Distribution of decay times}

Monte Carlo simulations which are run for varying postsynaptic receptor numbers (but for an otherwise identical population of synaptic boutons) are able to reproduce the distributions of amplitudes and rise times (see Fig. 4.15), but fail to account for the large range of variation observed in the distribution of decay-times: While the experimentally observed decay times are strongly fluctuating $(2.64 \pm 1.30 \mathrm{~ms}, \mathrm{CV}=0.49)$, the Monte Carlo simulations only yield a small fraction of this variability $(2.61 \pm 0.48 \mathrm{~ms}, \mathrm{CV}=0.18)$.

Furthermore the spectrum of decay times for a fixed number of receptors and constant vesicle content $(\mathrm{CV}=0.17$, Fig. 4.15) is left unchanged if variations in receptor number are included $(\mathrm{CV}=0.18$, Fig. 4.17) or if additionally the vesicle content is varied $(\mathrm{CV}=0.2$, Fig. 4.17). As shown above the distribution of decay times is hardly changed by a varying PSD size - despite the large effect on the distribution of rise times. Note that not only the mean value of $\tau_{\text {decay }}$, but also its $\mathrm{CV}$ does not depend on the receptor distribution. Hence the fact that mEPSCs are recorded from several synaptic boutons which might exhibit a wide spectrum of PSD shapes (see e.g., Walmsley et al., 1998) and receptor numbers (see above) does not account for the broad fluctuations observed in the distribution of decay times.

Due to individual morphological properties of single synaptic boutons it is possible that the time course of transmitter in the cleft is not the same for every synapse. We study the effect of fluctuations in the transmitter time course by varying the residence time of transmitter in the cleft $\langle T\rangle$ (Eq. 3.4). Leaving the extension of the postsynaptic density fixed we modulate the residence time $\langle T\rangle$ by varying the absorbing boundary $r_{\text {abs }}$ in our simulation model from run to run according to a Gaussian distribution with a mean of $530 \mathrm{~nm}$ and a standard deviation of $240 \mathrm{~nm}$, considering only values of $r_{\text {abs }}$ in between $150 \mathrm{~nm}$ and $1000 \mathrm{~nm}$. This is thought to roughly represent heterogenities in the synaptic morphology as well as in the morphology of the extra-synaptic space. As displayed in Fig. 4.19 fluctuations in the residence time of transmitter broaden the distribution of decay times: While residence times of $0.43 \pm 0.04 \mathrm{~ms}$ cause decay times of $2.61 \pm 0.48 \mathrm{~ms}$ $(\mathrm{CV}=0.18)$, the spectrum of decay times is larger $(2.66 \pm 1.32 \mathrm{~ms}, \mathrm{CV}=0.5)$ for larger fluctuations in the transmitter time course $(\langle T\rangle=0.4 \pm 0.14 \mathrm{~ms})$. The distributions of maximum amplitudes $(47.8 \pm 23.2$ channels vs. $45 \pm 21.3$ channels for fluctuating residence times) and rise times $(0.32 \pm 0.18 \mathrm{~ms}$ vs. $0.31 \pm 0.17 \mathrm{~ms}$ for fluctuating residence times) remain unchanged. We conclude that the broad fluctuations in the decay times of EPSCs can be explained by variations in the time course of glutamate in the cleft, corresponding to a mean residence time of glutamate in the cleft of $0.4 \pm 0.14 \mathrm{~ms}$. 


\section{- Experiment \\ - $<\mathrm{T}>=0.4+0.14 \mathrm{~ms}$ \\ $\cdots .<\mathrm{T}>=0.43+0.04 \mathrm{~ms}$}
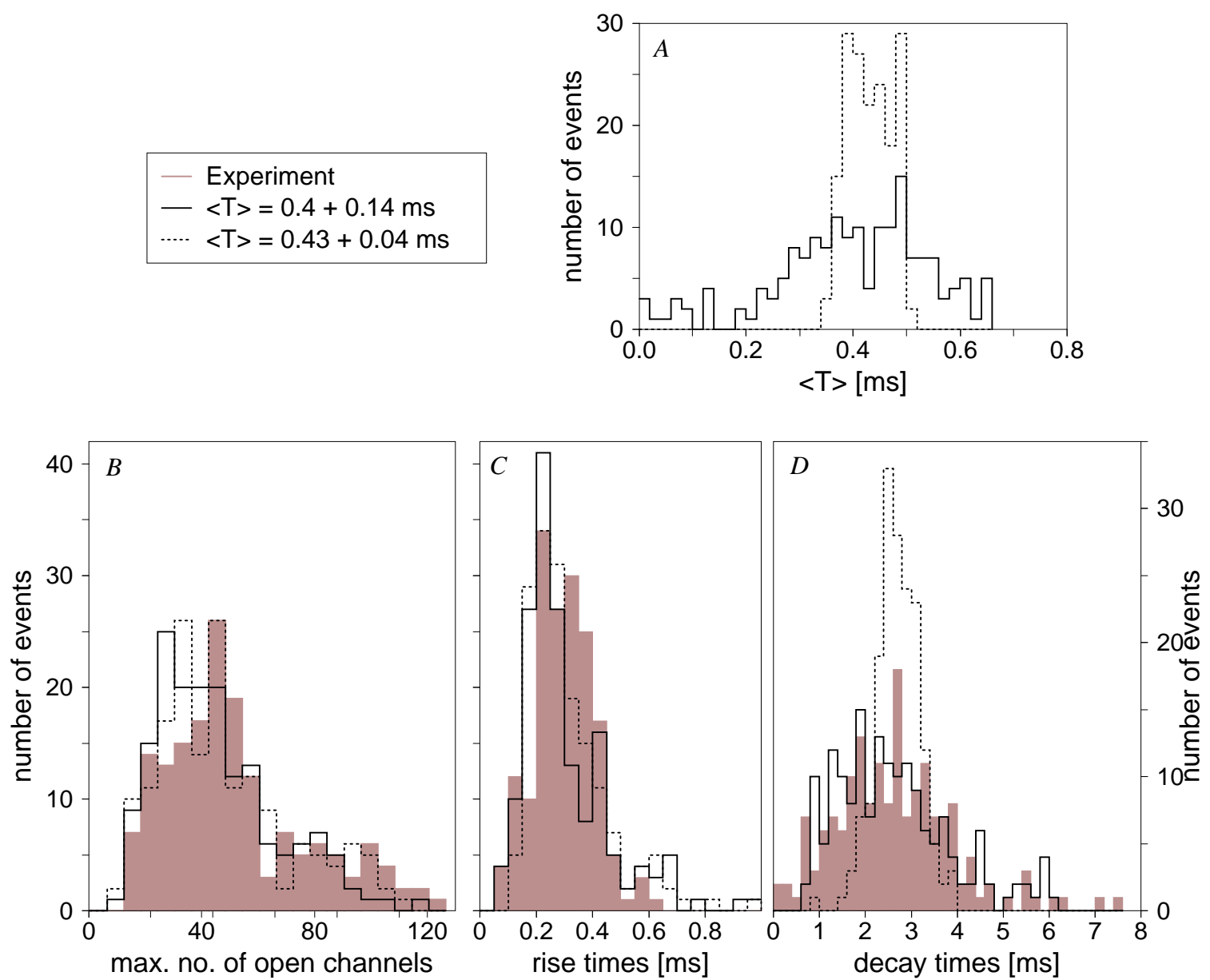

Figure 4.19.: Effect of fluctuations in the residence time $\langle T\rangle$ of transmitter $(A)$ on the distribution of amplitudes $(B)$, rise $(C)$ and decay times $(D)$; $\langle T\rangle=0.43 \pm 0.04 \mathrm{~ms}$ (white columns, dotted line), $\langle T\rangle=0.4 \pm 0.14 \mathrm{~ms}$ (white columns, solid line). Gray columns indicate the experimentally recorded miniature amplitudes. Monte Carlo simulations were run for synaptic boutons with varying absorbing boundaries $\left(\mathrm{r}_{\mathrm{abs}}\right.$ between 150 and $1000 \mathrm{~nm}$, mean of $\mathrm{r}_{\mathrm{abs}}=530 \pm 240 \mathrm{~nm}$ ), rest of parameters as in Tab.4.4. An amount of 4000 transmitter molecules is released at a release site randomly chosen within the PSD of radius $\mathrm{r}_{\mathrm{PSD}}=150 \mathrm{~nm}$. 


\subsection{Discussion}

The current chapter deals with modeling and understanding mechanisms of synaptic transmission that are located on the postsynaptic side. First we have introduced two kinetic models for calculating glutamate activated AMPA receptor mediated postsynaptic currents. The widely used seven-state model by Jonas et al. (1993) for hippocampal AMPA receptors has been illustrated, and a more compact three-state model for the receptor kinetics has been derived and fitted to experiments at brainstem interneurons.

On the basis of the given kinetic schemes we have used the complementary theoretical approaches of Monte Carlo simulations and local chemical kinetics to calculate glutamate mediated miniature EPSCs. The latter approach relies on the fact that at central synapses the transmitter molecules of a single vesicle provide an abundance of neurotransmitter for a low number of postsynaptic receptors. We have compared the two approaches in detail and shown their equivalence for average currents. The noisy character of the simulated EPSCs is apparent in broad distributions of amplitudes, rise and decay times of individual EPSCs and in good agreement with experiments at hippocampal synapses (Jonas et al., 1993). We have shown that for these synapses fluctuations in the time course of EPSCs are mainly due to the inherent noise in the receptor dynamics, whereas spatial fluctuations are less important. Despite the simplicity of our model it seems to comprise various relevant features of the transmission process to reproduce experimental data for average currents as well as the statistical properties of EPSCs.

We have used our model to study different receptor distributions, saturation and postsynaptic mechanisms for potentiation. Changes in the receptor distribution, e.g. clustering of receptors, do not efficiently potentiate postsynaptic signals, as long as the total number of receptors remains constant. An increase in the number of postsynaptic receptors or a change of receptor kinetics (Ambros-Ingerson and Lynch, 1993; Marienhagen et al., 1997) are found to be much more effective for potentiation.

Notice, that our Monte Carlo simulations of hippocampal synapses have lead to the conclusion that single channel noise is the major source of synaptic variability at these neurons. The situation seems different at brainstem interneurons where single channel noise fails to account for the whole spectrum of amplitudes and decay times. We have used Monte Carlo simulations to study these large fluctuations in the time course of mEPSCs at brainstem interneurons. We have shown that the release of a single vesicle activates about $70 \%$ of postsynaptic receptors, which also coincides with our estimates for hippocampal synapses. This implies a synaptic scenario where the release of a single vesicle yields an abundance of neurotransmitter in the cleft activating most of the postsynaptic receptors. Hence variations in vesicle content should not create a large effect on the postsynaptic synaptic signal. Our Monte Carlo simulations using a variable vesicle content underline this idea. We cannot find any significant effect, neither on the distributions of amplitudes, rise or decay times.

At the brainstem interneurons the large variability observed in the miniature amplitude distribution is explained by fluctuations in the number of postsynaptic receptors. The 
distribution of decay times however is not altered by fluctuations in receptor number, receptor arrangement or vesicle content, but reflects fluctuations in the residence time of the transmitter in the synaptic cleft. Most likely inhomogeneities in synaptic morphology, like morphological differences of extracellular space or inhomogeneous distributions of diffusion barriers in- and outside the cleft, are the reason for the fluctuations of the time course of transmitter in the cleft. The residence time of glutamate in the cleft at brainstem interneurons, which is able to account for the observed distribution of decay times, is estimated as $0.4 \pm 0.13 \mathrm{~ms}$. This is about half of the estimate found at hippocampal neurons.

Several studies (Edwards, 1995a; Lim et al., 1999; Oleskevich et al., 1999; Peters and Kaiserman-Abramof, 1969; Walmsley et al., 1998) have stressed that central synapses express a variety of PSD shapes and it has been assumed that the heterogeneity of the corresponding receptor distribution might contribute essentially to synaptic variability (Wahl et al., 1996). Our simulations do not support this idea. As demonstrated above a heterogeneity in the receptor distribution does not resolve the variability observed in amplitudes or decay times, but would broaden the distribution of rise times, which is not observed experimentally.

Central synapses are not only thought to exhibit a heterogeneity in PSD shape, but also in synaptic morphology, which has an effect on the transmitter dynamics inside the cleft: It has been suggested that the spreading of neurotransmitter inside the cleft is affected by the tortuosity of the cleft, by a dense staining, gel-like material, or by interaction of the diffusing molecules with receptors, transporters or other binding sites. Furthermore it is likely that structural inhomogeneities in the vicinity of a synapse, for instance local narrowing of intracellular gaps, can significantly retard diffusion and act like a diffusion barrier (see Chapter 3 for details and references).

Hence, the variability observed in the distribution of decay times may be due to fluctuations in the transmitter time course caused by a heterogeneity of synaptic morphology or may be due to variations in distribution, number or efficiency of uptake molecules. This question is addressed by bath application of the glutamate transporter blocker Daspartate, which at brainstem interneurons does not alter the EPSC kinetics. Control EPSCs do not differ from EPSCs recorded after inhibition of glutamate uptake with $1 \mathrm{mM}$ D-aspartate (Titz and Keller, 1997). We therefore conclude that glutamate transporters have little or no effect on the variability of mEPSCs. Rather differences in synaptic morphology as well as inhomogeneous distributions of diffusion barriers in- or outside the cleft are likely to be responsible for fluctuations in the transmitter dynamics.

We summarize that distributions of amplitudes, rise and decay times are shaped by independent properties of synaptic transmission. The distribution amplitudes indicates the spectrum of receptor numbers, the distribution of rise time the heterogeneity in PSD size and the distribution of decay times the fluctuations in the transmitter dynamics in the cleft - given the postsynaptic side is close to saturation after the release of individual vesicles. 


\section{Transmission properties of a single synaptic connection}

In this chapter we combine the results of the previous three chapters to design a theoretical model in order to study synaptic signal transduction and information-coding. It will be shown quantitatively that presynaptic mechanisms of vesicle release and postsynaptic receptor desensitization determine the range of synaptic ability to propagate rate-coded information depending on the type of nerve cell. For low release-probabilities often found at hippocampal synapses, the synaptic connection maintains the ability to transfer the rate at which input-stimuli arrive in a frequency-dependent manner towards the postsynaptic side. This is not the case for higher release-probabilities reported for instance at neocortical pyramidal cells.

Synaptic transmission can be understood as a sequence of three basic steps which transform an incoming presynaptic action potential (AP) into a postsynaptic signal: First, initiated by a presynaptic action potential (AP) vesicles fuse and release neurotransmitter into the synaptic cleft. Second, neurotransmitter spreads within the synaptic cleft and, third, interacts with postsynaptic receptors, which regulate the ionic flux through the postsynaptic membrane. This causes a detectable current, the excitatory postsynaptic current (EPSC) at the postsynaptic side, which we understand as the postsynaptic correlate of a presynaptic stimulus. We have modeled each step in this process in the previous chapters of this work. In Chapter 2 a model for presynaptic vesicle release and recruitment has been derived. In Chapter 3 it was shown that the transmitter motion within the synaptic cleft can be treated as a two-dimensional diffusive process, which is slowed down compared to free diffusion in aqueous solution. An estimate of the effective diffusion coefficient has been computed there. Finally it has been explained, how the opening behavior of receptors can be described by a kinetic three-state model and how postsynaptic currents are computed for a population of receptors within a single spine synapse (Chapter 4). Based on the models derived in these chapters we will now introduce an approach to compute the postsynaptic response for a given stimulus train.

We consider a synaptic scenario as displayed in Fig. 5.1: The axonal exit of the presynaptic neuron branches into a few collaterals at some distance from the cell forming individual synaptic connections with the postsynaptic side. These single synaptic boutons are activated by the same presynaptic stimulus and act as independent synaptic entities (Fig. 5.1). 


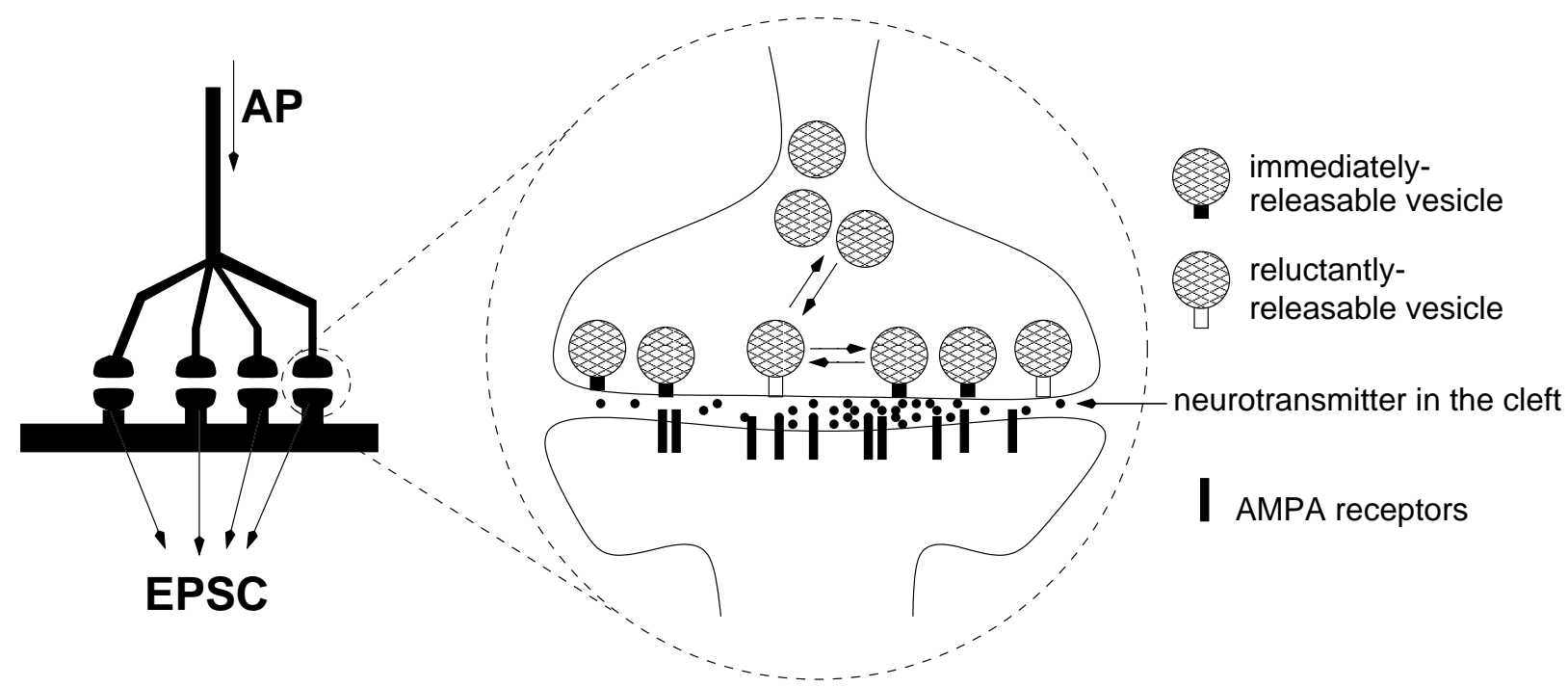

Figure 5.1.: Schematic picture of a synaptic connection. Here the axon of the presynaptic neuron branches into four collateral endings, which form individual synaptic contacts (= boutons) with the postsynaptic cell. Each synaptic bouton exhibits a single active zone, which contains a small number of immediatelyreleasable and reluctantly-releasable vesicles (as introduced in Chapter 2).

The currents from individual boutons add linearly[ and yield the total synaptic response, which will serve as our theoretical analogue of a stimulated postsynaptic current (EPSC).

Synapses differ in shape and size and this structural diversity is important for synaptic function. As many experiments have been carried out for glutamatergic synapses at hippocampal CA1 and CA3 cells (Allen and Stevens, 1994; Castro-Alamancos and Connors, 1997; Clements et al., 1992; Dobrunz et al., 1997; Dobrunz and Stevens, 1997; Hessler et al., 1993; Jonas et al., 1993; Pavlidis and Madison, 1999; Rosenmund et al., 1993; Schikorski and Stevens, 1997; Sorra and Harris, 1993; Spruston et al., 1995; Stevens and Tsujimoto, 1995) we will formulate our model for physiological conditions reported for hippocampal synapses. In the following we will provide a detailed description of the implementation of our model for the scenario of a small bouton-like hypothetical hippocampal synapse and briefly will summarize the employed parameters.

\footnotetext{
${ }^{1}$ If synaptic connections between a pair of neurons are spread across the dendritic tree, currents from individual boutons will be "filtered" due to the cable properties of the dendritic tree before being summed at or close to the cell soma (Rall and Agmon-Snir, 1998). For now, however, we ignore filtering effects, not only because synaptic contacts between pairs of hippocampal CA3 cells seem to be located on the same order of dendritic branch (Pavlidis and Madison, 1999), but also to keep the number of additional parameters (as e.g. input-resistance, membrane capacity and dendritic geometry) of our model low.
} 


\subsection{Model and Methods}

\section{Number of synaptic contacts}

Estimates about numbers of synaptic contacts between hippocampal cells are not known. It was suggested that axons occurring in stratum radiatum form a small number of two to five multiple contacts onto hippocampal CA1 target cells (Sorra and Harris, 1993). For our simulations we will assume five individual synaptic boutons, which are activated by the same presynaptic stimulus and contribute to the postsynaptic response. Each of the synaptic boutons operates as an independent entity, accessing its own synaptic resources. Notice, that in our approach the synaptic boutons do not interact. Furthermore we do not consider a morphological or physiological heterogeneity between the synaptic boutons.2 Therefore a larger number of active synaptic boutons simply increases the overall EPSC amplitude and reduces the relative noise in the recorded EPSC spectrum (data not shown here).

\section{Presynaptic mechanisms}

Here we briefly summarize experimental findings at hippocampal synapses and demonstrate that they roughly fit with observations made at the calyx of Held (see Chapter 2 for details). This rationalizes our approach of implementing the kinetic model for release and replenishment of presynaptic vesicles, as well as calcium related facilitation of release and dependency on the extracellular calcium concentration, which has been derived in Chapter 2.

Release-probabilities display a heterogeneity at hippocampal synapses (Rosenmund et al., 1993; Hessler et al., 1993; Murthy et al., 1997) and a simple depletion model of presynaptic vesicle recruitment could not account for the behavior during steady-state behavior (Fisher et al., 1997; Zucker, 1999). Recovery from depletion has been measured to occur with a time constant between 3 and 10 s (Dobrunz et al., 1997; Stevens and Tsujimoto, 1995). Facilitation of release-probability depends on calcium and occurs during paired-pulse recordings within a time window of 30 to $130 \mathrm{~ms}$ (Castro-Alamancos and Connors, 1997). Anatomical studies show that hippocampal synaptic boutons exhibit a single active zone, which is of the same size as the postsynaptic density right opposite the synaptic cleft (Harris and Sultan, 1995; Schikorski and Stevens, 1997; Sorra and Harris, 1993). On average eight vesicles are docked close to the membrane in that region (Schikorski and Stevens, 1997). This number fits with estimates of the readily-releasable pool of vesicles gained by electrophysiological measurements (Dobrunz et al., 1997; Dobrunz and Stevens, 1997; Rosenmund and Stevens, 1996). We implement the following presynaptic scenario (parameters from set 1 in Tab. 2.1): At rest on average 7.8 out of

\footnotetext{
${ }^{2}$ It has been shown in the in Sec. 4.5 that the variability in hippocampal miniature currents can be traced back to the intrinsic noise in the receptor dynamics. This result does not point towards a large (functional) morphological diversity.
} 
a total of 10 release-sites are filled with reluctantly-releasable (release-probability $w_{1}$ ) or immediately-releasable vesicles (release-probability $w_{2}, w_{2}>w_{1}$ ). The ratio of occupancy at rest between the two pools is chosen (on average) as $1: 13 / 7$ (see Chapter 2, set 1 of parameters in Tab. 2.1). Upon presynaptic stimulation vesicles are released according to the assigned release-probabilities $w_{1}$ and $w_{2}$.

As explained in detail in Chapter 2 and Appendix A the release-probabilities depend, first, on the extracellular calcium concentration, which is accounted for by a MichaelisMenten type dependence of the calcium flux on the extra cellular calcium concentration (Eq. 2.11). Second, release is assumed to be triggered by local domains of high calcium in the vicinity of a release-site, which are altered by changes in the global, spatially averaged presynaptic calcium concentration according to Eq. 2.13 and Appendix A. The global calcium increases due to stimulation as given by Eqs. 2.15 and 2.16. Hence for a given stimulus protocol we calculate the change in presynaptic calcium and estimate the release-probabilities $w_{1}$ and $w_{2}$. By comparing the release-probability with a random number it is decided whether the respective vesicle is released (from a randomly chosen point source within the active zone).

\section{Transmitter-dynamics in the cleft}

Individual release-sites are randomly chosen within the active-zone, which has the same lateral extension as the postsynaptic density. If a release event occurs all transmitter molecules are released at once from a single point. The transmitter molecules spread within the two-dimensional synaptic cleft with an effective diffusion constant $D_{\text {net }}$ according to Fick's law. After having crossed the absorbing boundary at radius $r_{\text {abs }}$ the transmitter molecules are irreversibly being removed (see Fig. 3.1 for a model of the synaptic cleft). The absorbing boundary comprises the effects of transporters and glutamate uptake by extra-synaptic mechanisms on the transmitter dynamics. The diffusion of transmitter in the cleft is characterized by the effective diffusion coefficient $D_{\text {net }}$, which is reduced compared to free diffusion in aqueous solution. We have given an estimate of $D_{\text {net }}$ on basis of measurements of the time course of glutamate in the cleft (Clements et al., 1992; Clements, 1996; Spruston et al., 1995) in Chapter 3. These experiments have been carried out at hippocampal synapses and lead to an estimate of the diffusion coefficient of $D_{\text {net }} \sim 20-50 \mathrm{~nm}^{2} / \mu$ s. Finally, the diffusion of single transmitter molecules is computed using a two-dimensional Langevin equation (Eq. 4.6). This approach is justified as preand postsynaptic terminal are separated by a very narrow cleft (see Fig. 1.2 and Chapter 3 for details).

\section{Postsynaptic receptor population}

The number of AMPA receptors on the postsynaptic side of a single synaptic bouton has been estimated as 60-70 (Spruston et al., 1995). Additionally glutamatergic synapses in the hippocampus express a small number of two to ten NMDA-channels (Spruston 
et al., 1995). These NMDA-channels are considered to be important for long-term changes (Buonomano and Merzenich, 1998; Malenka and Nicoll, 1999). Due to a voltagedependent magnesium block NMDA channels remain non-conducting for a membrane potential close to resting conditions. Consistently a block of NMDA receptors does not alter the response characteristics of short-term depression during repetitive stimulation (Castro-Alamancos and Connors, 1997; Markram et al., 1998b). Therefore we abstain from including NMDA receptors in our model for now.

As explained in Sec. 4.2 the population of postsynaptic AMPA receptors is modeled by Monte Carlo simulations based on kinetic master-equations. Transitions between the open, closed and desensitized state are computed by means of the kinetic three-state model introduced in Sec. 4.1.2. The respective kinetic rate constants have been estimated from patch-experiments at brainstem interneurons and are given in Tab. 4.2. The computed kinetic behavior of AMPA-receptors compares well results for hippocampal CA3 and CA1 cells (Jonas et al., 1993; Spruston et al., 1995) (see also Chapter đ).

The radius of the postsynaptic density (PSD) is set to $r_{\mathrm{PSD}}=150 \mathrm{~nm}$ as the average area of the PSD has been estimated as $\sim 0.04 \mu \mathrm{m}^{2}$ (Schikorski and Stevens, 1997). We assume that the population of AMPA receptors is homogeneously randomly distributed within the PSD and correspondingly the point of release for presynaptic vesicles is chosen at random within the range given by $r_{\mathrm{PSD}}=150 \mathrm{~nm}$.

\section{Computational procedure}

In each discrete time step $\Delta t$ of the Monte Carlo simulation a "new" distribution of transmitter molecules is generated, a possible change in the states of presynaptic releasesites as well as postsynaptic receptors is calculated and the number of open channels is computed for each synaptic bouton. The simulation time step $\Delta \mathrm{t}$ has been set to $5 \mu \mathrm{s}$, to account for the fastest time scales involved, which are the diffusion steps of individual transmitter molecules. The entire set of parameters is summarized in Tab. 5.1.

Figure 5.2 schematically illustrates the algorithm chosen to generate the overall synaptic response in terms of the number of open channels as a function of time or stimulus protocol, respectively. For a series of sweeps run for a set of given synaptic parameters but varying initializations of the random number generator, distributions of amplitudes are generated and compared to experiments. 


\begin{tabular}{|c|c|c|}
\hline Definition & Value & taken from \\
\hline number of synaptic boutons & 5 & (Sorra and Harris, 1993) \\
\hline release-sites per bouton & 10 & (Dobrunz and Stevens, 1997) \\
\hline $\begin{array}{l}\text { occupancy of release-sites at rest } \\
\text { (with immediately-releasable vesicles }\end{array}$ & $\begin{array}{l}78 \% \\
51 \%)\end{array}$ & Tab. 2.1 (set 1$)$ \\
\hline \multicolumn{2}{|c|}{$\begin{array}{l}\text { kinetic rate constants for vesicle-dynamics: } \\
\qquad \begin{array}{l}k_{r}=1.333 \mathrm{~s}^{-1}, k_{-r}=1.088 \mathrm{~s}^{-1} \\
k_{s}=0.163 \mathrm{~s}^{-1}, k_{t}=0.088 \mathrm{~s}^{-1}\end{array}\end{array}$} & $\begin{array}{l}\text { see Sec. 2.1, Eqs. } 2.7 \\
\text { from Tab. 2.1 }(\text { set } 1) \\
\text { from Tab. } 2.1(\text { set } 1)\end{array}$ \\
\hline $\begin{array}{l}\text { release-probabilities for vesicles: } \\
\text { initial: } w_{1}=0.1 \\
\text { full-facilitated: } w_{1}=0.25,\end{array}$ & $\begin{array}{l}1, w_{2}=0.4 \\
w_{2}=0.58\end{array}$ & $\begin{array}{l}\text { see Sec. 2.3.1, Eqs. } 2.13 \\
\text { from Tab. } 2.1 \text { (set } 1) \\
\text { from Tab. } 2.1 \text { (set } 1)\end{array}$ \\
\hline $\begin{array}{l}\text { dependency on extracellular calcium } \\
\text { change in global calcium after stimulat }\end{array}$ & & $\begin{array}{l}\text { Eq. 2.11, Tab. 2.1 (set } 1) \\
\text { Tab. 2.1 (set } 1)\end{array}$ \\
\hline radius of active zone $=$ radius of PSD $=$ & $=150 \mathrm{~nm}$ & (Schikorski and Stevens, 1997) \\
\hline AMPA-receptors per bouton & 70 & $($ Spruston et al., 1995) \\
\hline kinetic rate-constants (AMPA) & & Tab. 4.2 \\
\hline molecules per vesicle & 2000 & (Edwards, 1995a $)$ \\
\hline absorbing boundary & $500 \mathrm{~nm}$ & see Sec. 3.1 \\
\hline diffusion coefficient in the cleft & $40 \mathrm{~nm}^{2} / \mu \mathrm{s}$ & see Sec. 3.3 \\
\hline simulation time step & $5 \mu \mathrm{s}$ & \\
\hline
\end{tabular}

Table 5.1.: Parameters for Monte Carlo simulations of a single synaptic connection in the hippocampus. 


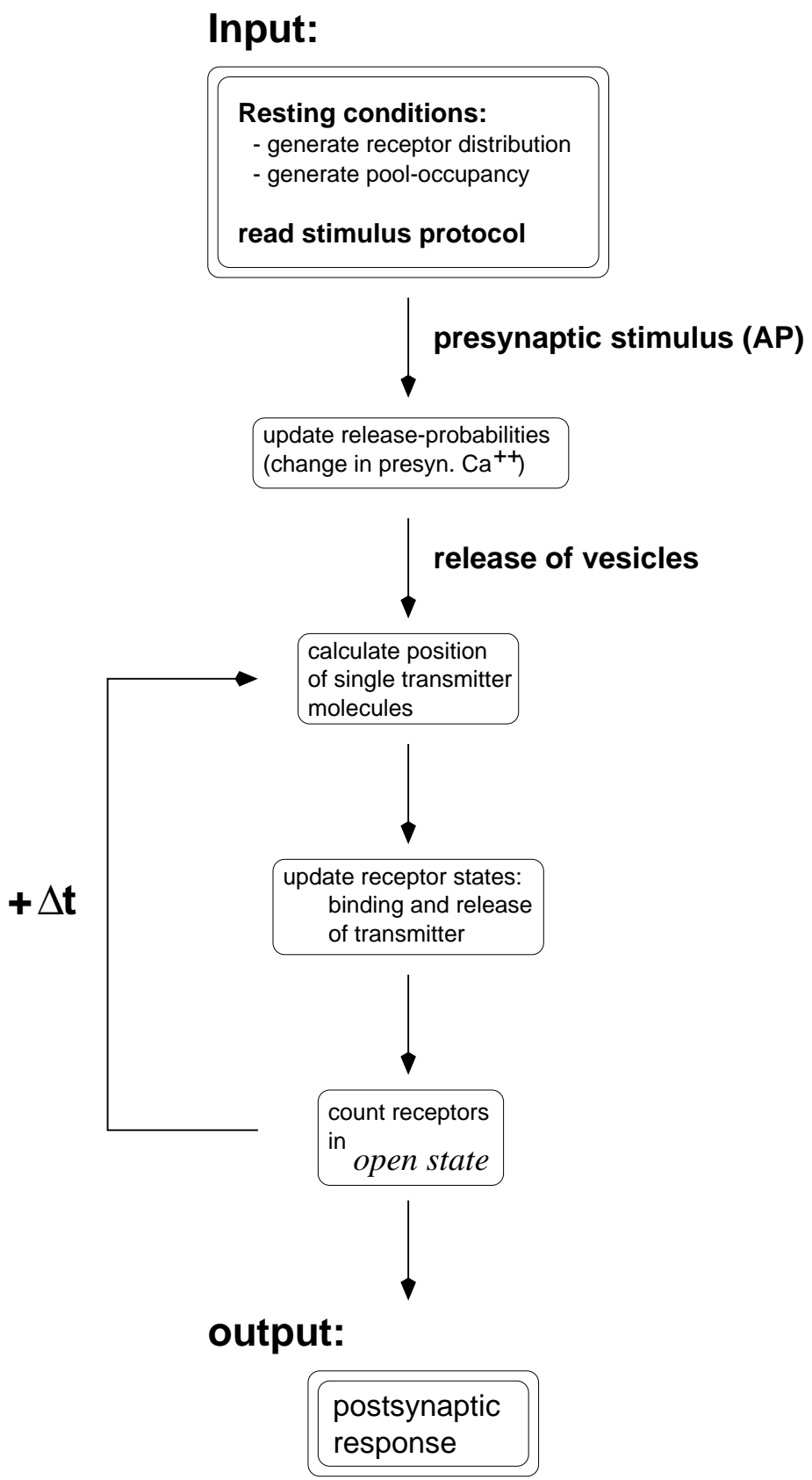

Figure 5.2.: Schematic picture of the algorithm to compute postsynaptic responses for given initial conditions and protocol of the applied presynaptic stimulation. The simulation steps are subsequently carried out during a single Monte Carlo sweep as indicated by arrows to compute the total current. This yields the number of open channels as function of time (for discrete simulation time steps $\Delta \mathrm{t}$ ) for a single synaptic bouton. The total response of a synaptic connection forming synaptic contacts via several boutons is computed for a sequence of stimuli by repeating the simulation procedure for each stimulus and with identical initial conditions and presynaptic stimulus protocol, but varying initializations of the random number generator for every synaptic bouton. The total synaptic response is gained by summing over the contributions of individual boutons. 


\subsection{Synaptic transmission of information}

The results in Chapter 2 promote the idea of rate coding to transmit presynaptic signals towards the postsynaptic side; i.e. the amplitude of postsynaptic currents during steadystate depression depends on the input frequency (Fig. 2.5D). This frequency-dependence of propagated presynaptic stimuli has been observed at synaptic connections in different areas of the brain (Abbott et al., 1997; Dobrunz and Stevens, 1997; Fisher et al., 1997; Galarreta and Hestrin, 1998; Markram et al., 1998b; Thomson and Deuchars, 1994). Although there is general agreement that the spike output by a neuron is correlated with the input to that neuron, the code by which the information is transmitted is not clear. One approach favors the idea that the mean firing-frequency or -rate, respectively, encodes the signal (= rate-coding), whereas an alternative view is that information is encoded in the precise times at which spikes occur (= temporal coding) (Rieke et al., 1997). Recently the focus of attention in this debate has been shifted towards a different point of view: It has been noted that the properties of synaptic transmission vary between specific types of neurons and these synaptic properties seem to determine the contributions of rate and temporal signals to the postsynaptic response (Tsodyks and Markram, 1997; Markram et al., 1998a; Varela et al., 1997).

Here we will follow the latter line of reasoning and address the question, how the underlying physiological mechanisms determine the properties of information transport at individual synapses. Due to the bursting activity of Schaffer/commissural projections hippocampal cells CA1 may receive repeated input with frequencies of up to $500 \mathrm{~Hz}$. Bursting patterns have been found to be unique at individual cells and to vary over a wide frequency range (Kandel and Spencer, 1968; Larson et al., 1986; Suzuki and Smith, 1985). We therefore assume that the frequency of presynaptic stimulus trains carries information and study how the corresponding rate code is propagated to the postsynaptic side. ${ }^{3}$

A phenomenological model of synaptic transmission, defined on the basis of electrophysiological experiments in the neocortex (Tsodyks and Markram, 1997; Varela et al., 1997), predicts a $1 / f$ decay in the amplitude of steady-state depression current $I_{\text {depr }}^{S}$ for stimulation frequencies $f$ larger than a limiting frequency $f_{\text {lim }}$, such that

$$
f>f_{\text {lim }}: \quad I_{\text {depr }}^{S} \sim \frac{1}{f} .
$$

Notice, that a $1 / f$ behavior of $I_{\mathrm{depr}}^{S}$ implies that $I_{\mathrm{depr}}^{S} / \tau$ is constant, i.e the charge entering the postsynaptic cell within a time window $\tau$ is constant. Hence for $f>f_{\text {lim }}$ the average postsynaptic depolarization will not change with increasing frequency, making a ratecoding of the input stimulus train impossible. Therefore the limiting frequency $f_{\text {lim }}$ sets

\footnotetext{
${ }^{3}$ In the present work we do not study how the presynaptic input relates to the generation of action potentials in the postsynaptic neuron. Instead we restrict our analysis to study the postsynaptic current as function of the presynaptic stimulus protocol. In that sense we use the term "information" to characterize, how the postsynaptic response reflects the presynaptic input pattern.
} 
the frequency range within which synapses are able to transmit information about the presynaptic firing rate.

Experiments at neocortical pyramidal neurons have shown the predicted $1 / f$-behavior for a range of stimulation frequencies in between 10 to $40 \mathrm{~Hz}$ (Tsodyks and Markram, 1997). Variations of release-probability by changes in extracellular calcium concentrations have caused a shift of $f_{\text {lim }}$ (Castro-Alamancos and Connors, 1997; Dobrunz et al., 1997; Markram et al., 1998a; Tsodyks and Markram, 1997), implying that the response characteristics of a synaptic connection not only depends on the neuronal type, but also on the specific physiological conditions. We employ our model of synaptic transmission to learn more about the primary determinants of signaling between central neurons and subsequently study the implications of synaptic mechanisms for the transmission of information.

In the following we discuss the results of Monte Carlo simulations of a synaptic connection between pairs of hippocampal pyramidal cells for two types of frequently used experimental stimulus protocols: First, two stimuli, i.e. paired-pulses, are applied for inter-pulse intervals of various duration (see Fig. 5.3) and the change in amplitude due to the repeated stimulation is studied. Second, a long lasting stimulus train is applied with a constant frequency (Fig. 5.5) in order to study the behavior during steady-state depression and to determine the limiting frequency $f_{\text {lim }}$.

\subsection{Results}

As shown in Figs. 5.4 and $5.5 \mathrm{~A}$ computer experiments for the same set of parameters but different initializations of the random number generator yield postsynaptic responses of large variability during paired-pulse as well as repetitive stimulation. Therefore steadystate currents during repetitive stimulation are computed by first averaging 16 synaptic responses during steady-state depressionf within an individual stimulus train and additionally taking the mean over 20 simulation runs. As shown in Fig. 5.5 presynaptic stimuli sometimes fail to trigger the release of a single vesicles and leave the postsynaptic side silent. These failures reflect the stochasticity of individual release events. Low averaged steady-state amplitudes during repetitive stimulation are hence partly caused by an increase in presynaptic failure rates.

\section{Paired-pulse recordings}

Responses of paired pulse stimulation exhibit a strong correlation between the amplitude of the first EPSC and the paired-pulse ratio (= amplitude of second stimulus / amplitude of first stimulus), which is also observed in experiments (Dobrunz and Stevens, 1997;

\footnotetext{
${ }^{4}$ We assume that after the application of the fifth stimulus the postsynaptic response has reached steady-state depression.
} 


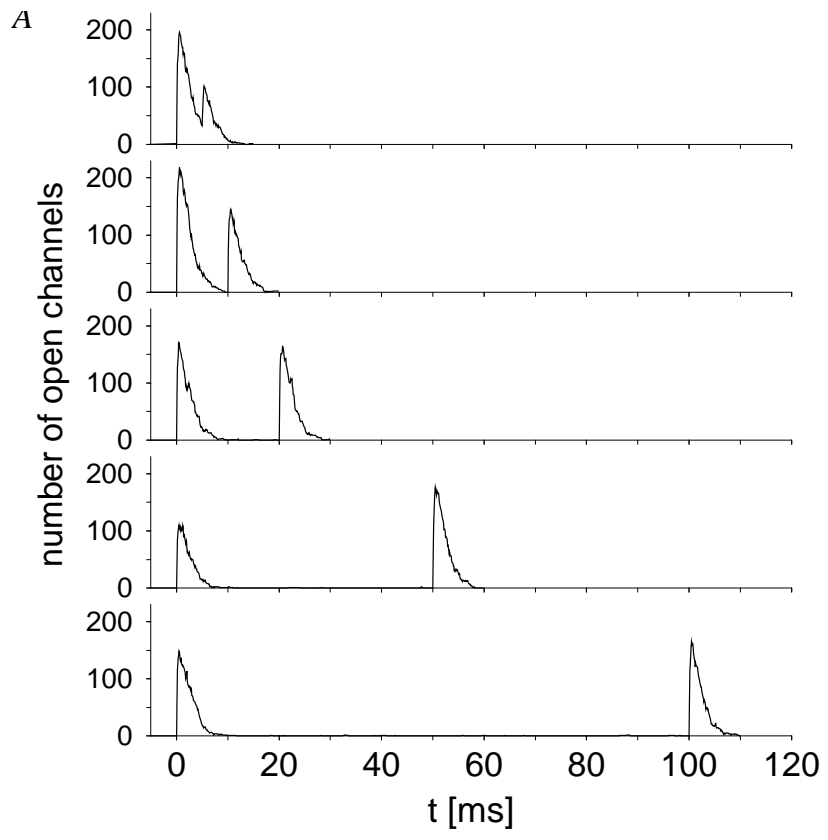

Figure 5.3.: Simulated paired-pulse recordings. Traces of individual simulation runs for differing inter-pulse intervals.

Pavlidis and Madison, 1999): As displayed in Fig. 5.4 EPSCs with a small first amplitude are followed by a large second response, whereas the response after a large first amplitude is smaller. We interpret this effect as "competition" for synaptic resources between the first and second EPSC.

It can be seen in Fig. $5.4 B$ that the paired-pulse ratio (PPR) for a shorter inter-pulse interval (IPI) of $10 \mathrm{~ms}$ on average is smaller than for an inter-pulse interval of $50 \mathrm{~ms}$. Since the time-scale of vesicle recruitment is of the order of $\sim 5 \mathrm{~s}$, paired-pulse responses should not exhibit any difference for an inter-pulse interval of $50 \mathrm{~ms}$ or $10 \mathrm{~ms}$, if the correlation was solely due to presynaptic depletion of vesicles. But the time constant for AMPA receptors to return from the desensitized into the unblocked state has been estimated as $\tau_{\text {res }} \sim 50$ to $100 \mathrm{~ms}$ (Barbour et al., 1994; Jonas et al., 1993; Spruston et al., 1995). Hence the small difference in PPRs for $10 \mathrm{~ms}$ and $50 \mathrm{~ms}$ IPIs is due to receptor desensitization, whereas the major component causing the correlation between the first response and the PPR is the depletion of vesicles.

\section{Depression during repetitive activity}

To further study pre- and postsynaptic contributions to depression, simulations of repetitive presynaptic stimulation are performed under block of desensitization of AMPA receptors, which is achieved by setting the desensitization rate $k_{d}$ to zero (see Tab. 4.2). For stimulation frequencies below $50 \mathrm{~Hz}$ EPSC amplitudes during steady-state depression 

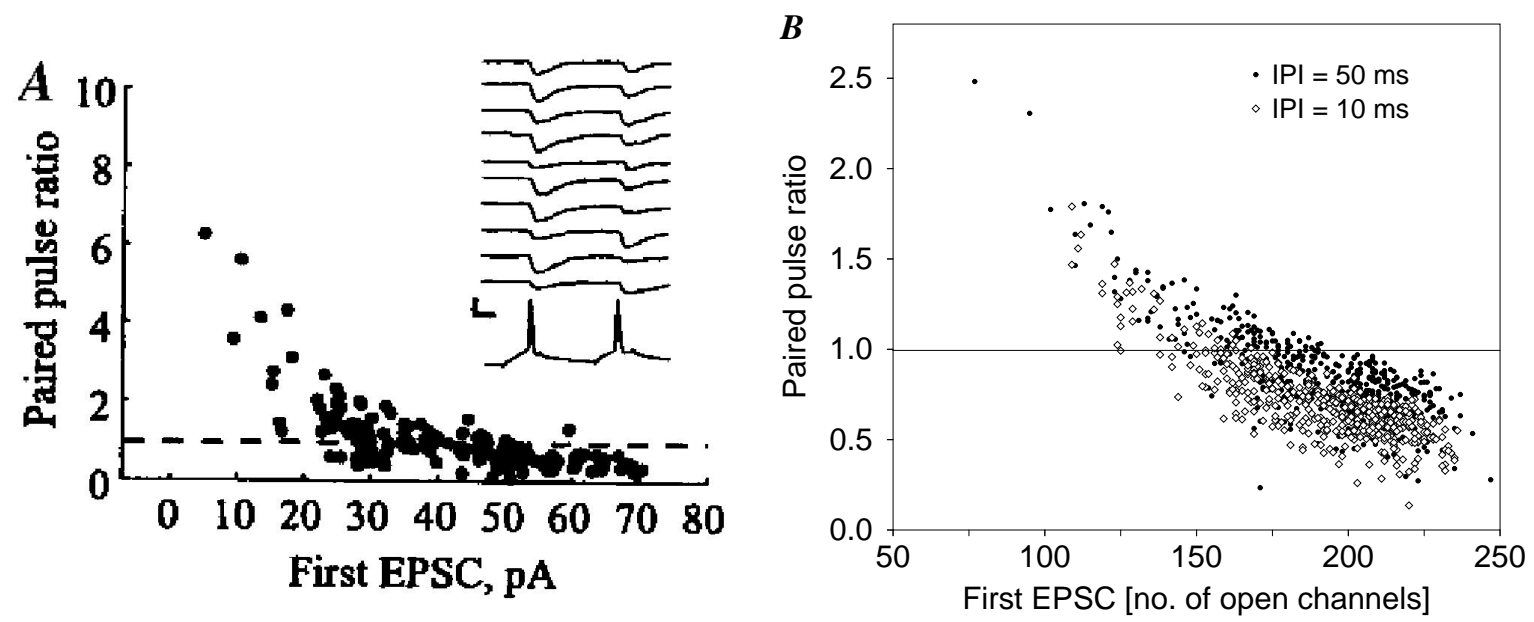

Figure 5.4.: Paired-pulse recordings in experiment and theory. $A$ : Paired-pulse recordings from experiments at hippocampal CA3 pyramidal cells for an inter-pulse interval of $50 \mathrm{~ms}$ (from Pavlidis and Madison, 1999). (Paired pulse ratio: amplitude of second EPSC / amplitude of first EPSC) B: Paired-pulse ratio as function of corresponding first EPSC. Results from 500 runs for identical parameters (Tab. 5.1) and inter-pulse intervals of $50 \mathrm{~ms}$ (black circles) and $10 \mathrm{~ms}$ (white circles).

do not differ from normal "physiological" conditions if receptor desensitization is blocked (Fig. 5.6), suggesting that depletion of releasable vesicles is the reason for depression at these frequencies. At stimulation frequencies above $50 \mathrm{~Hz}$ steady-state depression currents are considerably larger under block of desensitization indicating that depression is shaped to some extend by receptor desensitization.

As mentioned above the limiting frequency $f_{\text {lim }}$ has been introduced to indicate the upper limit of presynaptic input rates that can be transmitted towards the postsynaptic side by a frequency-dependent code. This means, that for frequencies larger than $f_{\text {lim }}$ the steady-state depression current $I_{\text {depr }}^{S}$ decays according to $1 / f$. We find a $1 / f$ decay in $I_{\text {depr }}^{S}$ only under certain conditions: Our simulations over a broad range of stimulation frequencies (Fig. 5.7) indicate a crucial dependence on the physiological properties of the individual neuronal connection. As shown in Fig. 5.7 for alterations in release-probability (by variations in extracellular calcium concentration) the predicted $1 / f$-behavior occurs at high presynaptic release-probabilities for frequencies $f>f_{\text {lim }}=35 \mathrm{~Hz}$. For lower releaseprobabilities or block of receptor desensitization, $I_{\text {depr }}^{S}$ decays according to a power law with an exponent larger than -1, provided the stimulation frequencies are between 10 and $200 \mathrm{~Hz}$. This algebraic dependence of $I_{\mathrm{depr}}^{S}$ on $f$ ends for frequencies higher than $200 \mathrm{~Hz}$ when the time-courses of individual EPSCs start to overlap and add to increasing steady-state values (see also Fig. 5.5B). 


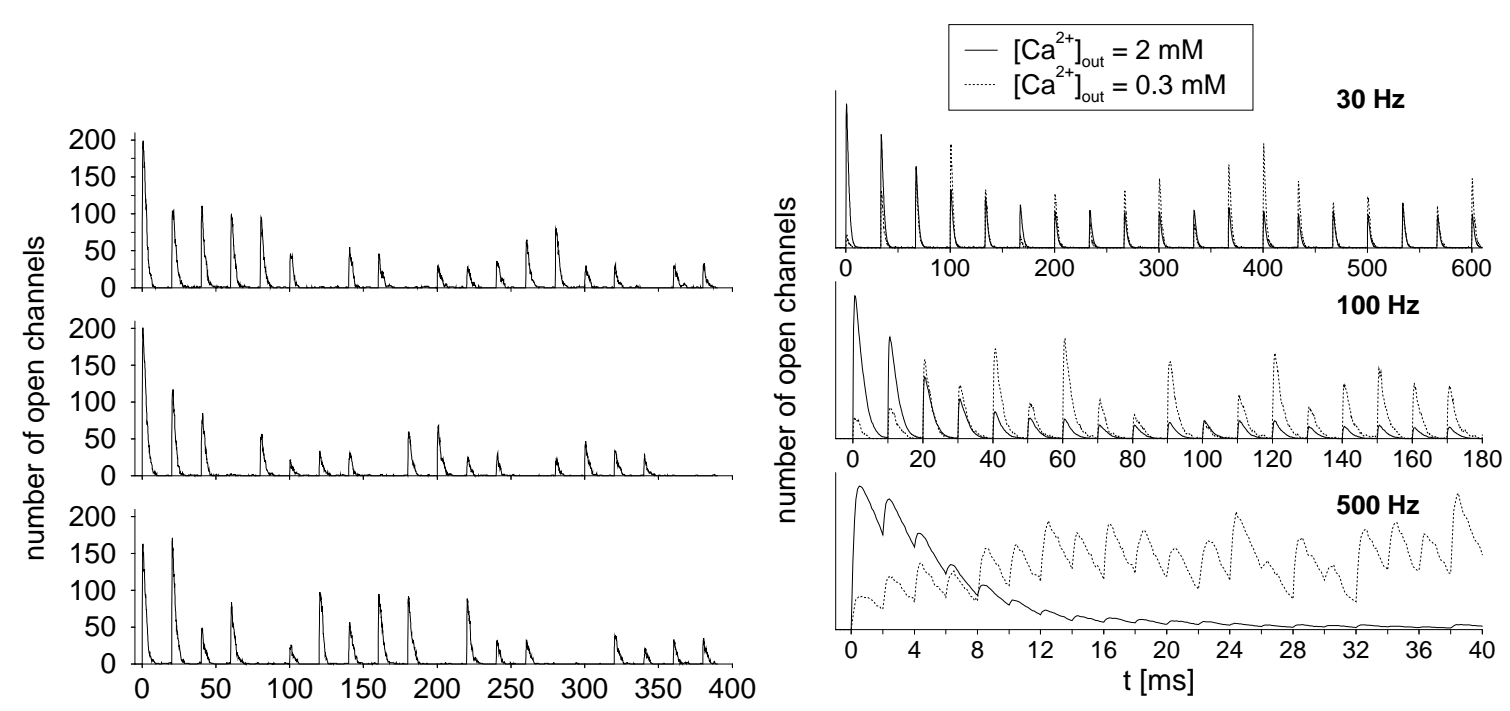

Figure 5.5.: Simulated postsynaptic responses during repetitive stimulation. A: Synaptic responses generated by three individual simulation runs for repetitive stimulation with $50 \mathrm{~Hz}$. B: Averaged synaptic responses (over 50 simulation runs each) for repetitive stimulation with 30,100 and $500 \mathrm{~Hz}$ and variations in extracellular calcium concentration (solid line: $\left[\mathrm{Ca}^{2+}\right]_{\text {out }}=2 \mathrm{mM}$; dotted line: $\left.\left[\mathrm{Ca}^{2+}\right]_{\text {out }}=0.3 \mathrm{mM}\right)$.

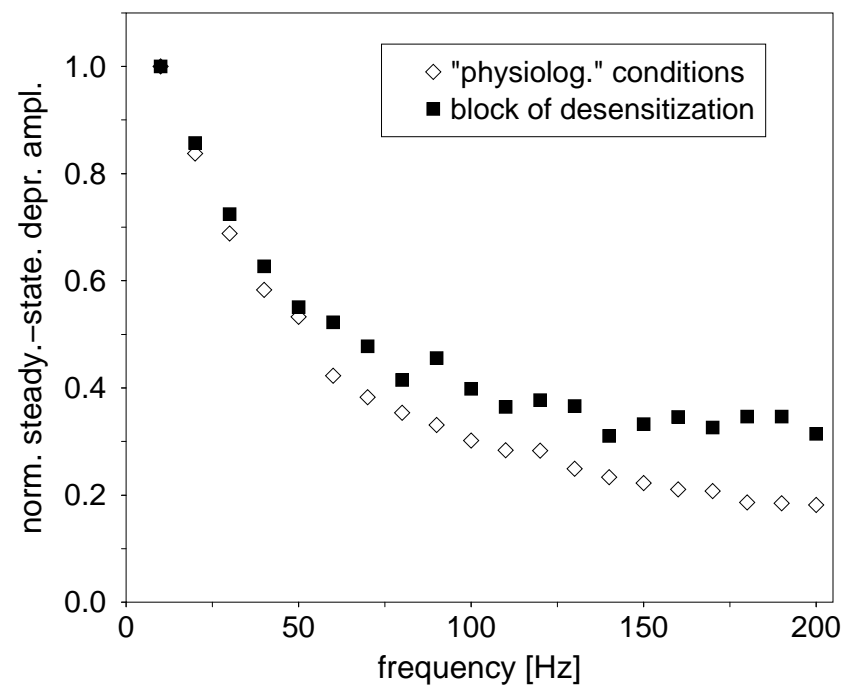

Figure 5.6.: Simulated postsynaptic response during repetitive stimulation. EPSC amplitude during steady-state depression (normalized by steady-state EPSC amplitude at $10 \mathrm{~Hz}$ ) as function of stimulation frequency for the set of parameters in Tab. 5.1 (white diamonds). Black squares indicate responses, if desensitization has been blocked by setting the rate $k_{d}$ (in Tab. 4.2) to zero. Individual data points are generated by averaging the 16 steady-state responses and computing the mean of 20 simulation runs. 


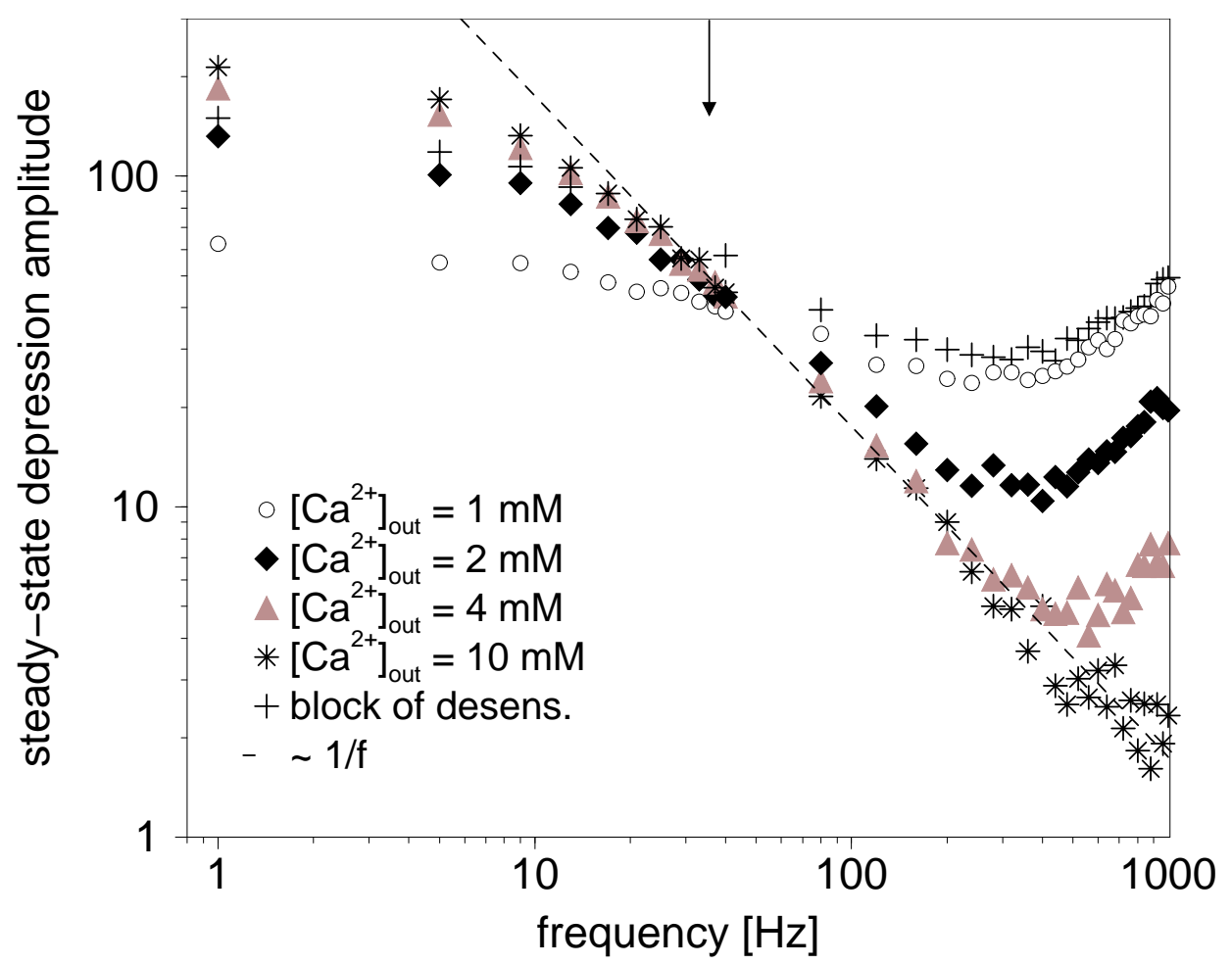

Figure 5.7.: Simulated postsynaptic response during repetitive stimulation. Number of open channels during steady-state depression as function of stimulation frequency for different extracellular calcium concentrations (white circles: $\left[\mathrm{Ca}^{2+}\right]_{\text {out }}=1 \mathrm{mM}$, black diamond: $\left[\mathrm{Ca}^{2+}\right]_{\text {out }}=2 \mathrm{mM}$, grey triangle: $\left[\mathrm{Ca}^{2+}\right]_{\text {out }}=4 \mathrm{mM}$, black star: $\left.\left[\mathrm{Ca}^{2+}\right]_{\text {out }}=10 \mathrm{mM}\right)$ and block of desensitization (parameters as in Fig. 5.6). The dashed line indicates a decay $\sim 1 / f$, and the arrow denotes the limiting frequency $f_{\text {lim }}$, where $I_{\text {depr }}^{S}$ approaches a $1 / f$-behavior for $\left[\mathrm{Ca}^{2+}\right]_{\text {out }}=4 \mathrm{mM}$. Individual data points are generated by averaging the 16 steady-state responses and computing the mean of 20 simulation runs. 


\subsection{Discussion}

Our simulations of a single synaptic connection for hippocampal pyramidal cells have produced two major results. First, we have demonstrated that synaptic-depression during repeated stimulation is generated by exhaustion of synaptic resources. For low frequencies $(<50 \mathrm{~Hz})$ EPSC amplitudes decrease consistently without and under block of receptor desensitization and hence decay due to the depletion of presynaptic vesicles in conjunction with a slow recovery process. In contrast depression arises as a joint effect of pre- and postsynaptic processes for higher stimulus frequencies. S Second, it has been shown that during repetitive stimulation the synaptic connection remains transmitting with a steadystate current $I_{\text {depr }}^{S}$. This steady-state current decreases with increasing stimulus-frequency and for physiological conditions does not reach a limiting frequency $f_{\text {lim. }}$. For elevated release-probabilities a $1 / f$-behavior is observed and limits the possible range of rate-coded transmission of presynaptic input patterns for frequencies $f$ larger than $f_{\lim }=35 \mathrm{~Hz}$ (Fig. 5.7).

This is in contrast to experimental findings at neocortical pyramidal neurons where a $1 / f$ dependence has been observed under physiological conditions for frequencies between 10 and $40 \mathrm{~Hz}$ (higher frequencies have not been studied experimentally), such that $f_{\lim }=$ $10 \mathrm{~Hz}$ and input-signals within this frequency-range being transmitted independently of the stimulus-frequency (Tsodyks and Markram, 1997). In the following paragraph we study the differences between the conditions at hippocampal neurons and those at neocortical neurons and discuss the implications of these differences on the responsecharacteristics of synapses.

On the basis of the simple depletion model the initial release probability has been estimated as $0.5 \pm 0.23$ at synapses between neocortical pyramidal neurons and facilitates to a value of $0.69 \pm 0.18$ (Tsodyks and Markram, 1997). This release-probability is higher than reported for hippocampal synapses, where average release-probabilities from 0.35 (Dobrunz and Stevens, 1997) to 0.1 (Hessler et al., 1993) have been measured. Consistent values have been implemented in our model, resulting in averaged release-probability from both pools of $w_{\mathrm{av}}=0.295$ under physiological extracellular calcium concentrations $\left(\left[\mathrm{Ca}^{2+}\right]_{\text {out }}=2 \mathrm{mM}\right)$, reaching $w_{\mathrm{av}}=0.59$ for elevated extracellular calcium of $4 \mathrm{mM}$ (see Fig. 2.4). Hence concerning the initial probability of release, our simulation results using $\left[\mathrm{Ca}^{2+}\right]_{\text {out }}=4 \mathrm{mM}$ (gray triangles in Fig. 5.7) can be compared to the findings at

\footnotetext{
${ }^{5}$ Paired-pulse experiments with inter-stimulus intervals of a few microseconds indicate that at these short times synaptic plasticity is shaped by additional mechanisms of release-dependent "lateral inhibition" of consecutive release, release inactivation and history-dependent enhanced facilitation for low releaseprobabilities (Dobrunz et al., 1997). At high stimulation frequencies of several hundred Hertz these mechanisms might considerably contribute to and shape the postsynaptic response. Nevertheless, possible physiological correlates of these effects are not clear and have not been included into our model.

${ }^{6}$ In the simple depletion model every stimulus depletes the pool of readily-releasable vesicles by a constant fraction, while the pool is simultaneously replenished with a single (slow) time constant (Liley and North, 1953).
} 


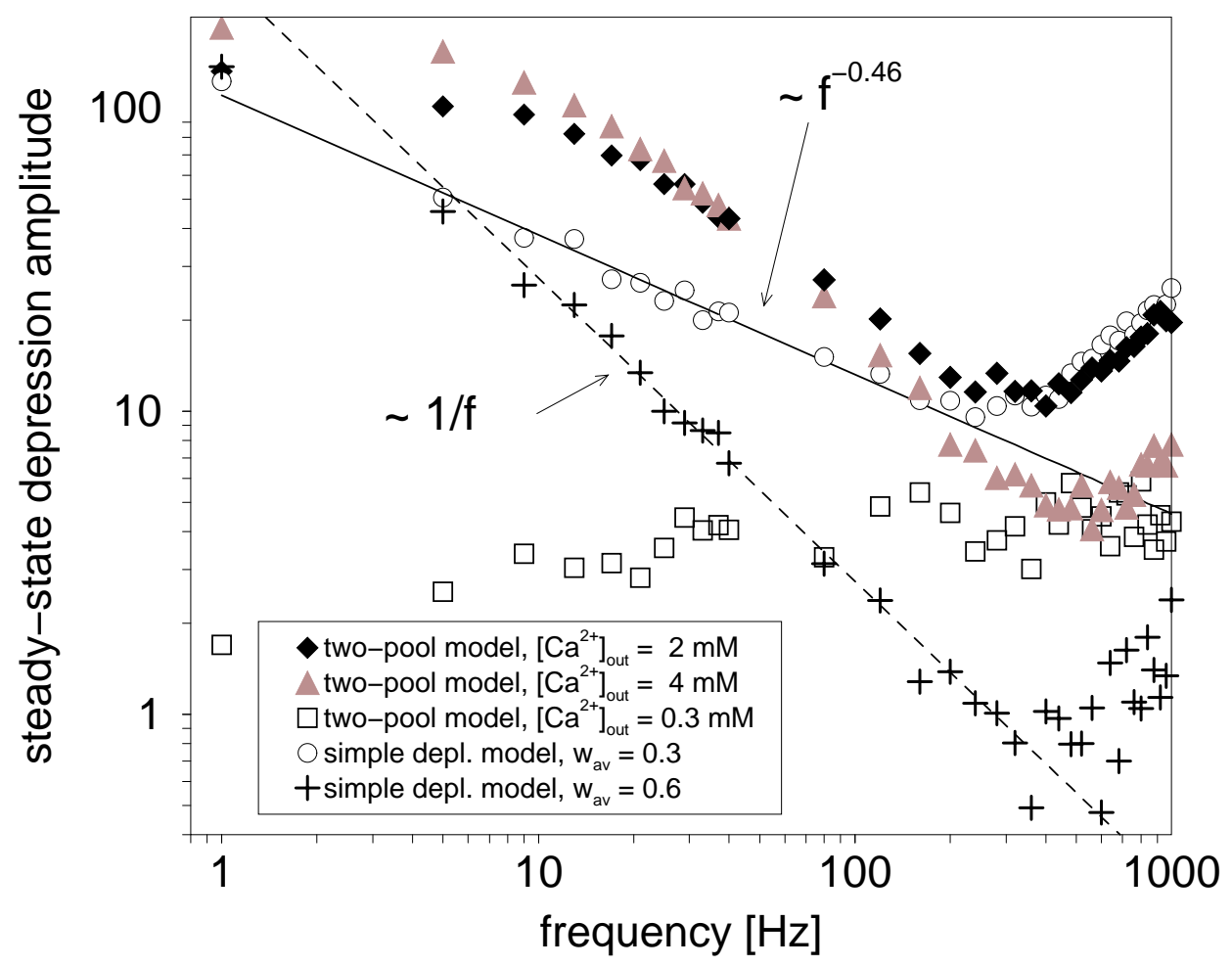

Figure 5.8.: Simulated postsynaptic response during repetitive stimulation. Number of open channels during steady-state depression as function of stimulation frequency. The two-pool model of presynaptic release of vesicles with two different release-probabilities is compared with a simple depletion model for normal extracellular calcium concentrations of $\left[\mathrm{Ca}^{2+}\right]_{\text {out }}=2 \mathrm{mM}$ (two-pool model: black diamonds, simple depletion model: white circles) and elevated release at $\left[\mathrm{Ca}^{2+}\right]_{\text {out }}=4 \mathrm{mM}$ (two-pool model: grey triangles, simple depletion model: black crosses). Parameters of the simple depletion model have been adjusted to yield the same release for the first stimulus (rest of parameters from Tab. 5.1). The black line indicates a fit to the simple depletion model that yields an exponent of -0.46 for $w_{\mathrm{av}}=0.3$ and of -1 for $w_{\mathrm{av}}=0.6$. For reduced $\left[\mathrm{Ca}^{2+}\right]_{\text {out }}=0.3 \mathrm{mM}$ the synapse facilitates, white squares indicating the steady-state facilitation. Individual data points are generated by averaging the 16 steady-state responses and computing the mean of 20 simulation runs. 
neocortical synapses and indeed display a $1 / f$ decay for frequencies larger $f_{\text {lim }}=35 \mathrm{~Hz}$.

Our model does not only differ from the "theoretical" neocortical synapse by Tsodyks and Markram (1997) by assuming lower release probabilities under physiological conditions, but also by modeling presynaptic dynamics differently. In order to compare the two approaches, we have replaced our presynaptic model for release of vesicles from two pools (Chapter 2) with the simple depletion model and have adjusted the two models for corresponding average initial release-probability $\left(w_{\mathrm{av}}=0.295\right)$. It can be seen in Fig. 5.8, that depression is stronger for the simple depletion model, but again does not exhibit a $1 / f$-behavior of the depression amplitude, i.e. decays with an exponent smaller than one $\left(\sim f^{-0.46}\right)$. But for higher release-probabilities $\left(w_{\mathrm{av}}=0.6\right)$ the decay of the steady-state depression current approaches $1 / f$ for $f>f_{\text {lim }}=4 \mathrm{~Hz}$. In contrast the limiting frequency for the presynaptic two-pool model is given by $f_{\text {lim }}=35 \mathrm{~Hz}$ for corresponding releaseprobabilities when $\left[\mathrm{Ca}^{2+}\right]_{\text {out }}=4 \mathrm{mM}$ (Fig. 5.8). This finding indicates that the additional pool of reluctantly-releasable vesicles not only assures larger postsynaptic responses, but also allows the transmission of a broader range of stimulus frequencies.

Alterations in release-probability not only determine the possible range for rate-coded transmission of stimulus frequencies, but additionally may turn a depressing synapse into a facilitating connection. The synaptic connection shown in Fig. 5.5 depresses under physiological conditions, but exhibits a facilitating behavior if the release-probabilities are lowered. For low extracellular calcium concentrations of $\left[\mathrm{Ca}^{2+}\right]_{\text {out }}=0.3 \mathrm{mM}$ the presynaptic release-probabilities are strongly reduced and hence the two pools of readily-releasable vesicles are only weakly depleted by individual stimuli. This leaves a sufficient amount of readily-releasable vesicles to be accessed by the facilitated release-probabilities originating from the accumulation of presynaptic residual calcium during repetitive stimulation. Therefore the steady-state activity during repeated stimulation rises with increasing frequency (Fig. 5.8). This finding is in accordance with recordings at neocortical pyramidal cells where differences between depressing and facilitating synaptic connections have been traced back to differing values of synaptic efficacy (Markram et al., 1998b).

We summarize that the specific combination of release-probability, receptor desensitization and presynaptic release-machinery determines whether synaptic connections facilitate or depress and set the range of input-rates, i.e. frequencies $f<f_{\text {lim }}$, that can be transmitted towards the postsynaptic side. 


\section{Conclusion and outlook}

The primary objective of this work has been the design of a model of synaptic transmission, which for a given presynaptic input-pattern computes the postsynaptic response. We have addressed this goal by successively developing theoretical descriptions for those physiological pre- and postsynaptic mechanisms that we consider to contribute crucially to the transmission process. In doing so we have been guided by results of physiological experiments at central synapses, which have led us to the theoretical implementation of the following synaptic scenario:

Upon arrival of an action potential in the presynaptic terminal two different types of vesicles release their contents of neurotransmitter into the synaptic cleft. The two kinds of vesicles differ regarding their release-probability and rates of recruitment. More reluctantlyreleasable vesicles are replenished faster with a time constant of $\sim 0.3 \mathrm{~s}$ as compared to immediately-releasable vesicles (time constant of refilling 5 to $6 \mathrm{~s}$ ). We have demonstrated that the specific dynamics of replenishment for both types of vesicles creates an activityinduced intrinsic, i.e. calcium-independent, extra-recruitment of reluctantly released vesicles. During repetitive presynaptic stimulation this mechanism ensures a steady-state activity, which is maintained at a higher level than explained by simple depletion of a single type of vesicles. Other features of presynaptic dynamics that are resolved by our model are the slow and calcium-independent recovery from depression within a few seconds, heterogeneities in release-probability and the calcium related facilitation mechanisms of release (von Gersdorff et al., 1997; Schneggenburger et al., 1999; Weis et al., 1999; Wu and Borst, 1999). As these patterns are also observed for presynaptic processes in other areas of the brain (Dobrunz and Stevens, 1997; Hessler et al., 1993; Murthy et al., 1997; Rosenmund et al., 1993; Zucker, 1999) we suggest that our model comprises some general features of presynaptic vesicle dynamics.

Once the transmitter-content of a vesicle is released into the cleft it spreads according to Fick's law. Pre- and postsynaptic terminal are closely attached in central synapses. We have shown that the transmitter dynamics can be effectively modeled by a twodimensional diffusion process, where absorbing boundary conditions reflect the effect of extra-synaptic transmitter uptake by transporters and diffusion into extra-synaptic space. We have demonstrated that transmitter diffusion in the cleft is slowed down compared to aqueous solution and have computed an estimate of the effective diffusion coefficient.

On the postsynaptic side the neurotransmitter interacts with receptors, which we have modeled by kinetic Markov models. Guided by a commonly used seven-state model for 
glutamate activated AMPA receptors (Jonas et al., 1993) we have introduced a simplified three-state model and fitted it to experiments performed at brainstem interneurons (Titz and Keller, 1996; Titz and Keller, 1997). The kinetic receptor models have been implemented in Monte Carlo simulations of a population of individual receptors in a synaptic bouton, which on the basis of Langevin and master equations comprise the dynamics of individual transmitter molecules, the transmitter-receptor interaction and the stochasticity of the receptor dynamics. The noisy character of the simulated EPSCs is apparent in broad distributions of amplitudes, rise and decay times of individual EPSCs and compares well with experiments at hippocampal synapses (Jonas et al., 1993). Our theoretical analysis has led to the conclusion that the distributions of amplitudes, rise and decay times are each shaped by independent characteristics of synaptic transmission: The distribution of amplitudes reflects variations in receptor number, the distribution of rise times maps the heterogeneity in PSD size and the distribution of decay times is largely determined by fluctuations in the transmitter dynamics in the cleft — given, the postsynaptic side is close to saturation after the release of an individual vesicle. This is the case for the two neuronal types of synapses studied here (in the hippocampus and brainstem), where the release of a single vesicle activates about seventy percent of the postsynaptic receptors. Additionally a model of local chemical kinetics has been designed to study the effect of different receptor distributions and of postsynaptic mechanisms for potentiation. Postsynaptic signals are found to be only weakly modified by changes in receptor distribution, but are more effectively potentiated by increasing receptor numbers.

We have finally combined our theoretical approaches of presynaptic vesicle dynamics, transmitter motion in the cleft and its interaction with postsynaptic receptors to create a model of a single synaptic connection between two neurons. For a given input stimuluspattern our model computes the postsynaptic current. We have implemented the physiological conditions for hippocampal synapses and studied the transmission properties of this specific connection. During repetitive stimulation the synapse has been shown to depress due to depletion of presynaptic vesicles and receptor desensitization, the latter only significantly contributing for stimulation frequencies higher than $50 \mathrm{~Hz}$. It has been illustrated how the transmission properties for synaptic connections in the hippocampus differ from those at pyramidal cells in the cortex (Tsodyks and Markram, 1997). While at hippocampal synapses rate-coded information is propagated in a frequency-dependent manner for a wide range of input-frequencies, at pyramidal synapses only frequencies below $10 \mathrm{~Hz}$ can be transmitted towards the postsynaptic side. We have demonstrated that the differences between the transmission properties of both types of synaptic connections are resolved by the lower release-probabilities at hippocampal synapses and by the deviations of our presynaptic two-pool model of release from the simple depletion of vesicles. Synapses which depress under physiological conditions have been shown to turn into facilitating connections if the average release-probability is strongly lowered. We can conclude that for a given synaptic contact the synaptic ability to transmit information is determined by the cooperative effects of the specific physiological conditions at the preand postsynaptic side. 
Our model of synaptic transmission opens a new path to understanding the importance of physiological mechanisms for the transmission of information at synaptic contacts between pairs of neurons. It has been suggested that the diversity observed in the structure and morphology of central synapses is related to function and different computational tasks of the corresponding neuronal connections (Edwards, 1995a; Walmsley et al., 1998). Our theoretical approach provides a chance to further elucidate this idea and to study transmission properties as function of specific synaptic morphology.

We have studied the properties of synaptic connections between neurons in the hippocampus which under physiological conditions depress during repetitive stimulation. But also the opposite effect, a facilitation of synaptic strength due to repeated activity, is observed at other neuronal connections. Even the same axon of a neuron was found to establish depressing contacts with a postsynaptic neuron and facilitating contacts at the same time with another (Markram et al., 1998b). We have demonstrated that one major difference between facilitating and depressing synapses is the presynaptic release-probability, suggesting that facilitating synapses exhibit very low release-probabilities $(\sim 0.05)$ about an oder of magnitude smaller than those at depressing synapses. Nevertheless, the detailed contributions to facilitation on the pre- and postsynaptic side remain unclear and could be studied by our model.

Very low release-probabilities come along with extreme failure-rates and hence make the transmission of information unreliable. The consequences of strongly fluctuating synaptic responses for the transmission process are unclear. For instance hippocampal pairedpulse recordings at a single connection yield paired-pulse ratios higher or lower than one for identical stimulation protocols, i.e. synaptic responses that randomly facilitate or depress (Fig. 5.4). If it matters for the processing of information, whether signals are transmitted via depressing or facilitating synaptic connections, synaptic responses either need to be averaged over unrealistically long observation periods or over many identical synaptic inputs. Noise and its implications for information-coding have been discussed in the frame of neuronal-network models and it has been suggested that the intrinsic neuronal noise determines the possible range of temporal and rate-coding of information (Plesser and Gerstner, 2000; Schneidman et al., 1998; Shadlen and Newsome, 1994; Zador and Dobrunz, 1997). Our Monte Carlo model of synaptic transmission explicitly accounts for the stochastic dynamics of synaptic transmission and might further elucidate the effects of noise on the propagation and coding of information - in particular if additional sources of synaptic variability, such as the observed heterogeneity in presynaptic releaseprobabilities, receptor number or synaptic morphology are taken into consideration.

Currently we calculate postsynaptic currents, not potentials, because we consider the former to be sufficient for studying transmission properties of individual synaptic connections. To learn more about the contributions of (noisy) synaptic inputs to the overall neuronal response we will have to take into account that synaptic contacts may be formed throughout the whole dendritic tree at variable distances from the cell soma. Due to the cable properties of the dendritic tree synaptic signals will contribute with different weight to the total response. It is not clear whether the filtering of noisy synaptic signals will 
yield additional contributions to the synaptic variability or rather create the opposite effect, a smoothing of synaptic noise. The consequences of noisy synaptic inputs on the action potential generation in the postsynaptic neuron remain to be studied. 


\section{A. Release-sites, calcium microdomains and global residual calcium}

In this appendix we will demonstrate, how local calcium gradients, so called calcium microdomains, emerge around the mouth of a conducting calcium channel due to the interaction of calcium with endogenous mobile calcium buffers. On the basis of the calculated concentration profile around a single calcium channel we will compute an estimate of the calcium concentration at the release-sites of a presynaptic vesicle and demonstrate how the local calcium concentration is related to the spatially averaged global calcium concentration.

The approach described here is a summary of previous work presented in (Naraghi and Neher, 1997; Neher, 1986; Neher, 1998a).

\section{A.1. Concentration profile due to a single conducting calcium channel}

Let us consider a presynaptic terminal, where under resting conditions free calcium is present with a concentration of $\left[\mathrm{Ca}^{2+}\right]_{\text {global }}$ and at equilibrium with an endogenous mobile calcium buffer $\mathrm{B}$. Upon presynaptic stimulation calcium conducting channels open, giving way for calcium entering the cell. Inside the cell calcium $\mathrm{Ca}^{2+}$ interacts with a calcium buffer $B$ according to

$$
\mathrm{Ca}^{2+}+B \underset{k_{\text {off }}}{\stackrel{k_{\text {on }}}{\rightleftarrows}} \mathrm{CaB}
$$

with $k_{\text {on }}$ and $k_{\text {off }}$ denoting the calcium binding and unbinding rates and $\mathrm{CaB}$ the bound calcium-buffer complex.

Based on the kinetic scheme (A1) and the diffusive properties of calcium with diffusion constant $D_{\mathrm{Ca}}$, changes in the intracellular calcium concentration $c(\mathbf{x}, t)$ are described by

\footnotetext{
${ }^{1}$ It has been shown, that interactions of calcium with fixed calcium buffers are of no importance for the calculation of steady-state calcium-concentration profiles (Stern, 1992; Naraghi and Neher, 1997). Therefore effects of fixed calcium buffers will be neglected in this context
} 
the following diffusion-reaction equation,

$$
\frac{\partial}{\partial t} c(\mathbf{x}, t)=-k_{\text {on }} b_{\text {free }}(\mathbf{x}, t) c(\mathbf{x}, t)+k_{\text {off }} b_{\text {bound }}(\mathbf{x}, t)+D_{\mathrm{Ca}} \Delta c(\mathbf{x}, t)
$$

where $b_{\text {free }}(\mathbf{x}, t)$ is the free and $b_{\text {bound }}(\mathbf{x}, t)$ the bound buffer concentration, which evolve in time according to similar reaction-diffusion equations.

Calcium buffering occurs on a time scale of a few microseconds (Neher, 1986), which is fast compared to the time the calcium channel remains in the conducting state. Hence the flux through the point-like channel-pore (described as flux through a spherical half-shell for $r \rightarrow 0)$ may be assumed to be constant in time and is given by $i_{S} / 2 F\left(i_{S}\right.$ : single channel current and F: Faraday constant).

First we will consider the solution of Eq. A2 for a single open channel, located at the origin. In the steady-state the influx of calcium is modeled as a source term at the origin, i.e. the channel position. Hence we have to solve the following inhomogeneous, partial differential equation:

$$
0=-k_{\text {on }} b_{\text {free }}(\mathbf{x}) c(\mathbf{x})+k_{\text {off }} b_{\text {bound }}(\mathbf{x})+D_{\mathrm{Ca}} \Delta c(\mathbf{x})+\frac{i_{S}}{2 F} \delta(\mathbf{x}) .
$$

We compute the concentration profile for changes of calcium at the inside of the presynaptic membrane. For the sake of simplicity we will solve the two-dimensional form of Eq. A3 in oder to calculate the free calcium concentration for the planar case ${ }^{2}$ and a single channel located at the origin. Hence polar coordinates are adequate and the concentrations are taken to be independent of solid angle and to depend only on $r=|\mathbf{x}|$.

We distinguish between two kinds of buffers:

a) The first buffer is present in high concentration, so that changes in $b_{\text {free }}^{(1)}(r)$ due to calcium binding are small and can be neglected. In other words the concentration of free buffer is assumed to remain constant during synaptic stimulation and hence is given by the concentration at rest: $b_{\text {free }}^{(1)}(r) \sim\left[B^{(1)}\right]_{\mathrm{r}}$. To solve Eq. A2 we make the ansatz

$$
c(r)=C_{1}+\frac{C_{2}}{r} \exp (-r / \lambda)
$$

and apply the Laplacian

$$
\Delta \frac{\exp (-r / \lambda)}{r}= \begin{cases}\frac{\exp (-r / \lambda)}{r \lambda^{2}} & \text { for } r \neq 0 \\ 2 \pi \delta(r) & \text { for } r=0\end{cases}
$$

so that the three constants are determined by

$$
C_{1}=\left[\mathrm{Ca}^{2+}\right]_{\text {global }}, \quad C_{2}=\frac{i_{S}}{4 \pi F D_{\mathrm{Ca}}}, \quad \text { and } \quad \lambda=\sqrt{\frac{D_{\mathrm{Ca}}}{k_{\mathrm{on}}^{(1)}\left[B^{(1)}\right]_{\mathrm{r}}}} .
$$

\footnotetext{
${ }^{2}$ In a first approximation the two-dimensional formulation of Eq. A3 is justified because it has been shown that concentration changes are confined to a narrow shell in the immediate vicinity of the membrane, if a highly diffusive buffer is present in high concentration (Neher, 1986).
} 
We have chosen the boundary condition that $c(r)$ vanishes for large distances from the channel. The calcium concentration is infinite directly at the open calcium channel $(r=0)$, corresponding to a constant finite influx, and decays with the length constant $\lambda=\sqrt{D_{\mathrm{Ca}} / k_{\mathrm{on}}^{(1)}\left[B^{(1)}\right]_{\mathrm{r}}}$. The length $\lambda$ is determined by affinity and concentration of the endogenous buffer (see Eq. A4) and is of the order of a few tens of nanometers (Neher, 1998a). In order to estimate the constant $C_{1}$ it is assumed that the calcium-buffer interaction is in local equilibrium (Neher, 1986); in particular this implies that $\left[\mathrm{Ca}^{2+}\right]_{\text {global }}=$ $[\mathrm{CaB}] k_{\mathrm{off}} /\left(\left[B^{(1)}\right]_{r} k_{\mathrm{on}}\right)=C_{1}$.

b) The second buffer is in a state close to saturation by the global calcium concentration $\left[\mathrm{Ca}^{2+}\right]_{\text {global }}$ and reaches the saturated state rapidly, reducing the concentration of free buffer according to

$$
b_{\text {free }}^{(2)} \sim \frac{K_{D}^{(2)}\left[B^{(2)}\right]_{\text {tot }}}{\left[\mathrm{Ca}^{2+}\right]_{\text {global }}+K_{D}^{(2)}}
$$

where $K_{D}^{(2)}=k_{\text {off }}^{(2)} / k_{\text {on }}^{(2)}$ is the dissociation constant and $\left[B^{(2)}\right]_{\text {tot }}$ the total buffer concentration of $b^{(2)}$. For the same boundary conditions and assumption of local equilibrium as introduced above, the steady-state solution of Eq. A2 is given by

$$
c(r)=\left[\mathrm{Ca}^{2+}\right]_{\text {global }}+\frac{i_{S}}{4 \pi F D_{\mathrm{Ca}} r} \mathrm{e}^{-r / \lambda} \quad \text { with } \quad \lambda=\sqrt{\frac{D_{\mathrm{Ca}}\left(\left[\mathrm{Ca}^{2+}\right]_{\text {global }}+K_{D}^{(2)}\right)}{k_{\mathrm{on}}^{(2)}\left[B^{(2)}\right]_{\mathrm{tot}} K_{D}^{(2)}}} .
$$

If both buffers are present, then

$$
\frac{D_{\mathrm{Ca}}}{\lambda^{2}}=k_{\mathrm{on}}^{(1)}\left[B^{(1)}\right]_{r}+\frac{k_{\mathrm{on}}^{(2)}\left[B^{(2)}\right]_{\text {tot }} K_{D}^{(2)}}{\left[\mathrm{Ca}^{2+}\right]_{\text {global }}+K_{D}^{(2)}} .
$$

We denote by $\lambda_{0}=\sqrt{D_{\mathrm{Ca}} /\left(k_{\mathrm{on}}^{(1)}\left[B^{(1)}\right]_{r}\right)}$ the length over which $c(r)$ decays if only the highly concentrated buffer is present and introduce $\beta=k_{\mathrm{on}}^{(2)}\left[B^{(2)}\right]_{\mathrm{tot}} /\left(k_{\mathrm{on}}^{(1)}\left[B^{(1)}\right]_{r}\right)$ to rewrite $\lambda$ as

$$
\lambda=\lambda_{0}\left(1+\beta \frac{K_{D}^{(2)}}{K_{D}^{(2)}+\left[\mathrm{Ca}^{2+}\right]_{\text {global }}}\right)^{-1 / 2} .
$$

We furthermore assume the saturable buffer $b^{(2)}$ to be present in much lower concentration than buffer $b^{(1)}$, so that $k_{\text {on }}^{(2)}\left[B^{(2)}\right]_{\text {tot }} \ll\left(k_{\text {on }}^{(1)}\left[B^{(1)}\right]_{r}\right)$ (Neher, 1999).

This implies $\beta \ll 1$, so that we can expand the square-root and get

$$
\lambda=\lambda_{0}\left(1-\frac{1}{2} \frac{\beta}{1+\frac{\left[\mathrm{Ca}^{2+}\right]_{\text {global }}}{K_{D}^{(2)}}}\right) .
$$


It should be noted that this result describes the local calcium concentration in the vicinity of an open channel and depends on the global presynaptic calcium concentration. Due to presynaptic stimulation the global calcium concentration increases above resting level $\left(=\left[\mathrm{Ca}^{2+}\right]_{\mathrm{r}}\right)$ by an amount of $\Delta \mathrm{Ca}^{2+}$, which for a given stimulus protocol is calculated by means of the single compartment model described in Sec. 2.4. Calcium-buffer interactions equilibrate rapidly, so that the solution in Eq. A5 depends on the elevated global calcium concentration $\left[\mathrm{Ca}^{2+}\right]_{\text {global }}=\left[\mathrm{Ca}^{2+}\right]_{\mathrm{r}}+\Delta \mathrm{Ca}^{2+}$.

Inserting $\left[\mathrm{Ca}^{2+}\right]_{\text {global }}=\left[\mathrm{Ca}^{2+}\right]_{\mathrm{r}}+\Delta \mathrm{Ca}^{2+}$ into Eq. A5 yields the dependency of the microdomain calcium concentration $c(r)$ on the increase above resting calcium $\Delta \mathrm{Ca}^{2+}$,

$$
c\left(r, \Delta \mathrm{Ca}^{2+}\right)=\left[\mathrm{Ca}^{2+}\right]_{\mathrm{r}}+\frac{i_{S}}{4 \pi F D_{\mathrm{Ca}} r} \mathrm{e}^{-r / \lambda} \quad \text { with } \quad \lambda=\lambda_{0}\left[1-\frac{\gamma}{1+\frac{\Delta \mathrm{Ca}^{2+}}{K_{D}}}\right],
$$

with the constants $\gamma=\frac{\beta}{2} \frac{K_{D}^{(2)}}{K_{D}^{(2)}+\left[\mathrm{Ca}^{2+}\right]_{\mathrm{r}}}$ and $K_{D}=K_{D}^{(2)}+\left[\mathrm{Ca}^{2+}\right]_{\mathrm{r}}$.

\section{A.2. Calcium concentration at the release-site}

Synaptic vesicles are believed to be docked to release-sites which are embedded in regions of high density of $\mathrm{Ca}^{2+}$ channels (Llinas et al., 1992; Haydon et al., 1994) and may specifically be linked to one or several distinct $\mathrm{Ca}^{2+}$ channels at short distances (Bennett et al., 1992; Sheng et al., 1996; Rettig et al., 1997).

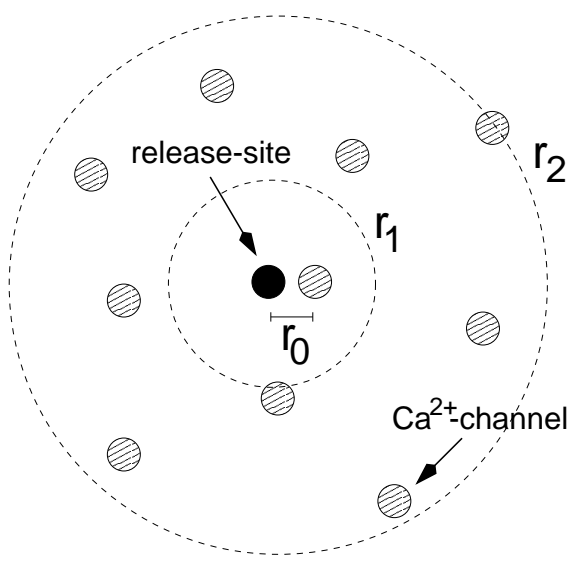

Figure A.1.: Geometric configuration of $\mathrm{Ca}^{2+}$-channels (hatched circles) around releasesite (black circle): A single $\mathrm{Ca}^{2+}$-channel at distance $r_{0}$ is colocalized with the release-site, which itself is surrounded by a cluster of $\mathrm{Ca}^{2+}$-channels (average area per channel $r_{1}^{2} \pi$, cluster-radius $r_{2}$ ).

Here we consider a presynaptic release-site at position $\mathbf{r}$, which is colocalized with a $\mathrm{Ca}^{2+}$ channel (at position $\mathbf{r}_{\mathbf{0}}$ ), and embedded in a cluster of $(n-1) \mathrm{Ca}^{2+}$-channels, which are 
distributed with a density of $\rho_{\mathrm{Ca}}=1 / r_{1}^{2} \pi\left(r_{1}\right.$ indicating the average distance between the channels) within a cluster radius of $r_{2}$ (see Fig. A.1).

We assume that buffer is present in the presynaptic terminal with a high concentration, such that $\lambda \ll r_{2}$ and that microdomains due to individual $\mathrm{Ca}^{2+}$ channels add up linearly (Neher, 1998a).

We consider the $n$ channels to be identical and open simultaneously. Then the calcium concentration at the release-site, $c(\mathbf{r})$, is given as the sum over the individual microdomains (Eq. A7) of all $n$ channels as

$$
c(\mathbf{r})=\left[\mathrm{Ca}^{2+}\right]_{\mathrm{r}}+\frac{i_{S}}{4 \pi F D_{\mathrm{Ca}}}\left[\frac{\mathrm{e}^{-\left|\mathbf{r}-\mathbf{r}_{0}\right| / \lambda}}{\left|\mathbf{r}-\mathbf{r}_{0}\right|}+\sum_{\nu=1}^{n-1} \frac{\mathrm{e}^{-\left|\mathbf{r}-\mathbf{r}_{\nu}\right| / \lambda}}{\left|\mathbf{r}-\mathbf{r}_{\nu}\right|}\right],
$$

with $\mathbf{r}_{\nu}$ denoting the position of the $\nu$ th channel. This expression simplifies, if we locate the release site at the origin, i.e. set $\mathbf{r}=0$ in the above equation.

The closest, possibly colocalized $\mathrm{Ca}^{2+}$ channel is at a distance $r_{0}$ from the release-site and contributes most to $c(r)$ and therefore is considered separately. For $r_{1} \gg r_{0}$ the sum is replaced by an integral (channel-density $\rho_{\mathrm{Ca}}=1 / r_{1}^{2} \pi$ ), so that

$$
\begin{aligned}
\sum_{\nu=1}^{n} \frac{\mathrm{e}^{-r_{\nu} / \lambda}}{r_{\nu}} \longrightarrow \quad & \rho_{\mathrm{Ca}} 2 \pi \int_{r_{1}}^{r_{2}} d r \mathrm{e}^{-r / \lambda} \\
& =\frac{2 \lambda}{r_{1}^{2}}\left(\mathrm{e}^{-r_{1} / \lambda}-\mathrm{e}^{-r_{2} / \lambda}\right) .
\end{aligned}
$$

Since $r_{2} \gg \lambda$, the second term can be neglected. Furthermore we consider $\lambda$ to be larger than $r_{1}$, i.e. $r_{1} / \lambda<1$, expand $\exp \left(-r_{1} / \lambda\right) \approx 1-r_{1} / \lambda$, and find

$$
\begin{aligned}
c(0)-\left[\mathrm{Ca}^{2+}\right]_{\mathrm{r}} & =\frac{i_{S}}{4 \pi F D_{\mathrm{Ca}}} \frac{1}{r_{0}}\left[1+\frac{2 \lambda r_{0}}{r_{1}^{2}}\right] \\
\text { Eq. } \mathrm{A7} & \frac{i_{S}}{4 \pi F D_{\mathrm{Ca}}} \frac{1}{r_{0}}\left[1+\frac{2 \lambda_{0} r_{0}}{r_{1}^{2}}\left(1-\frac{\gamma}{1+\frac{\Delta \mathrm{Ca}^{2+}}{K_{D}}}\right)\right],
\end{aligned}
$$

where $2 r_{0} / r_{1} \ll 1$ has been neglected during the second step.

For a fixed extracellular calcium concentration $\left[\mathrm{Ca}^{2+}\right]_{\text {out }}$ the influx of $\mathrm{Ca}^{2+}$ is given by $J_{\mathrm{Ca}}\left(\left[\mathrm{Ca}^{2+}\right]_{\text {out }}\right)=i_{S} / 2 F$ (see Eq. A3). Its dependency on $\left[\mathrm{Ca}^{2+}\right]_{\text {out }}$ has been studied experimentally (Church and Stanley, 1996; Schneggenburger et al., 1999) as discussed in Sec. 2.3.1. By introducing the constants $\alpha=1 / 2 \pi D_{\mathrm{Ca}} r_{0}$ and $\eta=2 \lambda_{0} r_{0} / r_{1}^{2}$, which will serve as free fit parameters of the model in Chapter 2, we find the local calcium concentration at the release-site $c(0)=\left[\mathrm{Ca}^{2+}\right]_{\mathrm{RS}}$ of Eq. 2.10 in Sec. 2.3.1

$$
\left[\mathrm{Ca}^{2+}\right]_{\mathrm{RS}}=\left[\mathrm{Ca}^{2+}\right]_{\mathrm{r}}+J_{\mathrm{Ca}}\left(\left[\mathrm{Ca}^{2+}\right]_{\mathrm{out}}\right) \alpha\left[1+\eta\left(1-\frac{\gamma}{1+\frac{\Delta \mathrm{Ca}^{2+}}{K_{D}}}\right)\right] .
$$





\section{Bibliography}

Abbott, L. F., Varela, J. A., Sen, K., and Nelson, S. B. (1997). Synaptic depression and cortical gain control. Science, 275:220-224.

Agmon, N. and Edelstein, A. L. (1997). Collective binding properties of receptor arrays. Biophys. J., 72:1582-1594.

Aharon, S., Parnas, H., and Parnas, I. (1994). The magnitude and significance of ca2+ domains for release of neurotransmitter. Bull. Math. Biol., 56:1095-1119.

Allen, C. and Stevens, C. F. (1994). An evaluation of causes for unreliability of synaptic transmission. Proc. Natl. Acad. Sci. USA, 91:10380-10383.

Ambros-Ingerson, J. and Lynch, G. (1993). Channel gating kinetics and synaptic efficacy: A hypothesis for expression of long-term potentiation. Proc. Natl. Acad. Sci. USA, 90:7903-7907.

Asztely, F., Erdemli, G., and Kullmann, D. M. (1997). Extrasynaptic glutamate spillover in the hippocampus: Dependence on temperature and the role of active glutamate uptake. Neuron, 18:281-293.

Bain, A. I. and Quastel, D. M. (1992). Multiplicative and additive ca(2+)-dependent components of facilitation at mouse endplates. J. Physiol. (Lond.), 455:383-405.

Barbour, B., Keller, B. U., Llano, I., and Marty, A. (1994). Prolonged presence of glutamate during excitatory synaptic transmission to cerebellar purkinje cells. Neuron, 12:1331-1343.

Bartol, T. M., Land, B. R., Salpeter, E. E., and Salpeter, M. M. (1991). Monte carlo simulation of miniature endplate current generation in the vertebrate neuromuscular junction. Biophys. J., 59:1290-1307.

Bekkers, J. M., Richerson, G. B., and Stevens, C. F. (1990). Origin of variability in quantal size in cultured hippocampal neurons and hippocampal slices. Proc. Natl. Acad. Sci. USA, 87:5359-5362.

Bekkers, J. M. and Stevens, C. F. (1990). Presynaptic mechanism for long-term potentiation in the hippocampus. Nature, 346:724-729. 
Bekkers, J. M. and Stevens, C. F. (1995). Quantal analysis of EPSCs recorded from small numbers of synapses in hippocampal cultures. J. Neurophysiol., 73:1145-1156.

Bennett, M. R., Calckos, N., and Scheller, R. H. (1992). Syntaxin: a synaptic protein implicated in docking of synaptic vesicles at presynaptic active zones. Science, 257:255-259.

Bennett, M. R., Farnell, L., Gibson, W. G., and Karunanithi, S. (1995). Quantal transmission at purinergic junctions: Stochastic interaction between ATP and its receptors. Biophys. J., 68:925-935.

Bennett, M. R., Farnell, L., Gibson, W. G., and Lavidis, N. A. (1997). Synaptic transmission at visualized sympathetic boutons: Stochastic interaction between acetylcholine and its receptors. Biophys. J., 72:1595-1606.

Bergles, D. E. and Jahr, C. E. (1997). Synaptic activation of glutamate transporters in hippocampal astrocytes. Neuron, 19:1297-1308.

Bertram, R., Sherman, A., and Stanley, E. F. (1996). Single-domain/bound calcium hypothesis of transmitter release and facilitation. J. Neurophysiol., 75:1919-1931.

Betz, W. J. (1970). Depression of transmitter release at the neuromuscular junction of the frog. J. Physiol. (Lond.), 206:629-644.

Bier, M., Kits, K. S., and Borst, J. G. (1996). Relation between rise times and amplitudes of GABAergic postsynaptic currents. J. Neurophysiol., 75:1008-1012.

Borst, J. G. G., Helmchen, F., and Sakmann, B. (1995). Pre- and postsynaptic whole-cell recordings in the medial nucleus of the trapezoid body of the rat. J. Physiol. (Lond.), 489:825-840

Borst, J. G. G., Lodder, J. C., and Kits, K. S. (1994). Large amplitude variability of GABAergic IPSCs in melano tropes from xenopus leavis: evidence that quantal size differs between synapses. J. Neurophysiol., 71:639-655.

Borst, J. G. G. and Sakmann, B. (1996). Calcium influx and transmitter release in a fast CNS synapse. Nature, 383:431-434.

Borst, J. G. G. and Sakmann, B. (1999). Effect of changes in action potential shape on calcium currents and transmitter release in a calyx-type synapse of the rat auditory brainstem. Phil. Trans. R. Soc. Lond. B., 354:355-363.

Boyd, I. A. and Martin, A. R. (1956). The end-plate potential in mammalian muscle. J. Physiol. Lond., 132:74-91.

Buonomano, D. V. and Merzenich, M. M. (1998). Cortical plasticity: from synapses to maps. Annu. Rev. Neurosci., 21:149-186. 
Busch, C. and Sakmann, B. (1990). Synaptic transmission in hippocampal neurons. Numerical reconstruction of quantal IPSCs. Cold Spring Harbor Symposia on Quantitative Biology, LV:69-80.

Castro-Alamancos, M. A. and Connors, B. W. (1997). Distinct forms of short-term plasticity at excitatory synapses of hippocampus and neocortex. Proc. Natl. Acad. Sci. USA, 94:4161-4166.

Chad, J. E. and Eckert, R. (1984). Calcium domains associated with individual channels can account for anomalous voltage relations of Ca-dependent responses. Biophys. J., 45:993-999.

Church, P. J. and Stanley, E. F. (1996). Single l-type calcium channel conductance with physiological levels of calcium in chick ciliary ganglion neurons. J. Physiol. (Lond)., 496:59-68.

Clements, J. D. (1996). Transmitter time course in the synaptic cleft: its role in central synaptic function. Trends Neurosci., 19:163-171.

Clements, J. D., Lester, R. A. J., Tong, G., Jahr, C. E., and Westbrook, G. L. (1992). The time course of glutamate in the synaptic cleft. Science, 258:1498-1501.

Colquhoun, D. and Hawkes, A. G. (1995). The principles of the stochastic interpretation of ion-channel mechanisms, pages 397-481. In (Sakmann and Neher, 1995).

del Castillo, J. and Katz, B. (1954). Quantal components of the end-plate potential. J. Physiol., 124:560-573.

den Loos, H. V. (1963). Fine structure of synapses in the cerebral cortex. Zeitsch. Zellforsch., 60:815-825.

Destexhe, A., Mainen, Z. F., and Sejnowski, T. J. (1994). Synthesis of models for excitable membranes, synaptic transmission and neuromodulation using a common kinetic formalism. J. Computat. Neurosci, 1:195-230.

Destexhe, A., Mainen, Z. F., and Sejnowski, T. J. (1998). Kinetic Models of Synaptic Transmission, pages 1-26. In (Koch and Segev, 1998).

Diamond, J. and Jahr, C. (1997). Transporters buffer synaptically released glutamate on a submillisecond time scale. J. Neurosc., 17:4672-4687.

Dittmann, J. S., Kreitzer, A. C., and Regehr, W. G. (2000). Interplay between facilitation, depression, and residual calcium at three presynaptic terminals. J. Neurosc., 20:13741385 .

Dobrunz, L. E., Huang, E. P., and Stevens, C. F. (1997). Very short-term plasticity in hippocampal synapses. Proc. Natl. Acad. Sci. USA, 94:14843-14847. 
Dobrunz, L. E. and Stevens, C. F. (1997). Heterogeneity of release probability, facilitation, and depletion at central synapses. Neuron, 18:995-1008.

Dodge, F. A. and Rahamimoff, R. (1967). Co-operative action of calcium ions in transmitter release at the neuromuscular junction. J. Physiol. (Lond.), 193:419-432.

Dzubay, J. A. and Jahr, C. E. (1999). The concentration of synaptically released glutamate outside of the climbing fiber-purkinje cell synaptic cleft. J. Neurosc., 19:5265-5274.

Eccles, J. C. and Jaeger, J. C. (1958). The relationship between the mode of operation and the dimensions of the junctional regions at synapses and motor end-organs. Proc. R. Soc. Lond. B. Biol., 148:38-56.

Edmonds, B., Gibbs, A. J., and Colquhoun, D. (1995). Mechanisms of activation of glutamate receptors and the time course of excitatory synaptic currents. Annu. Rev. Physiol., 57:495-519.

Edwards, F. A. (1995a). Anatomy and electrophysiology of fast central synapses lead to a structural model for long-term potentiation. Physiol. Rev., 75:759-787.

Edwards, F. A. (1995b). LTP - a structural model to explain the inconsistencies. Trends Neurosci., 18:250-255.

Edwards, F. A., Konnerth, A., and Sakmann, B. (1990). Quantal analysis of inhibitory synaptic transmission in the dentate gyrus of rat hippocampal slices: a patch clamp study. J. Physiol. (Lond.), 430:213-249.

Engert, F. and Bonhoeffer, T. (1999). Dendritic spine changes associated with hippocampal long-term synaptic plasticity. Nature, 399:66-70.

Faber, D. S., Young, W. S., Legendre, P., and Korn, H. (1992). Intrinsic quantal variability due to stochastic properties of receptor-transmitter interactions. Science, 258:14941498 .

Fisher, S. A., Fischer, T. M., and Carew, T. J. (1997). Multiple overlapping processes underlying short-term synaptic enhancement. Trends Neurosc., 20:170-177.

Fogelson, A. L. and Zucker, R. S. (1985). Presynaptic calcium diffusion from various arrays of single channels. implications for transmitter release and synaptic facilitation. Biophys. J., 48:1003-1017.

Forsythe, I. D. (1994). Direct patch recordings from identified presynaptic terminals mediating glutamatergic EPSCs in the rat CNS, in vitro. J. Physiol. (Lond.), 479:381-387.

Forti, L., Bossi, M., Bergamaschi, A., Villa, A., and Malgaroli, A. (1997). Loose-patch recordings of single quanta at individual hippocampal synapses. Nature, 388:874-878. 
Foster, T. C. and McNaughton, B. L. (1991). Long-term potentiation of CA1 synaptic transmission is due to increased quantal size, not quantal content. Hippocampus, 1:79-91.

Frerking, M., Borges, S., and Wilson, M. (1995). Variation in GABA mini amplitude is the consequence of variation in transmitter concentration. Neuron, 15:885-895.

Frerking, M. and Wilson, M. (1996). Saturation of postsynaptic receptors at central synapses? Curr. Opin. Neurobiol., 6:395-403.

Galarreta, M. and Hestrin, S. (1998). Frequency-dependent synaptic depression and the balance of excitation and inhibition in the neocortex. Nature Neuroscience, 1:587594.

Gardiner, C. W., editor (1983). Handbook of Stochastic Methods. Springer, Berlin Heidelberg New York Tokio.

Garthwaite, J. (1985). Cellular uptake disguises action of l-glutamate on n-methyl-daspartate receptors. Br. J. Pharmacol., 85:297-307.

Gingrich, K. J. and Byrne, J. H. (1985). Simulation of synaptic depression, posttetanic potentiation, and presyanptic facilitation of synaptic potentials from sensory neurons mediating gill-withdrawal reflex in Aplysia. J. Neurophysiol., 53:652-669.

Glavinovic, M. I. (1999). Monte Carlo simulation of vesicular release, spatiotemporal distribution of glutamate in synaptic cleft and generation of postsynaptic currents. Pflügers Arch., 437:462-470.

Hall, Z. W., editor (1992). An introduction to molecular neurobiology. Sinauer Associates, Sunderland Massachusetts.

Harris, K. M. and Kater, S. B. (1994). Dendritic spines: cellular specializations imparting both stability and flexibility to synaptic function. Annu. Rev. Neurosci., 17:341-371.

Harris, K. M. and Sultan, P. (1995). Variation in the number, location and size of synaptic vesicles provides an anatomical basis for the nonuniform probability of release at hippocampal CA1 synapses. Neuropharmacology, 34:77-95.

Häusser, M. and Roth, A. (1997). Dendritic and somatic glutamate receptor channels in rat cerebellar purkinje cells. J. Physiol. (Lond.), 501:77-95.

Haydon, P. G., Henderson, E., and Stanley, E. F. (1994). Localization of individual calcium channels at the release face of a presynaptic nerve terminal. Neuron, 13:12751280 .

Heidelberger, R., Heinemann, C., Neher, E., and Matthews, G. (1994). Calcium dependence of the rate of exocytosis in a synaptic terminal. Nature, 371:513-151. 
Heinemann, C., von Rüden, L., Chow, R. H., and Neher, E. (1993). A two-step model of secretion control in neuroendocrine cells. Pflügers Arch., 424:105-112.

Held, H. (1893). Die centrale Gehörleitung. Arch. Anat. Physiol., pages 201-248.

Helmchen, F., Borst, J. G. G., and Sakmann, B. (1997). Calcium dynamics associated with a single action potential in a CNS presynaptic terminal. Biophys. J, 72:14581471 .

Helmchen, F., Imoto, K., and Sakmann, B. (1996). Ca2+ buffering and action potentialevoked Ca2+ signaling in dendrites of pyramidal neurons. Biophys. J, 70:1069-81.

Hermann, H. J., Wolf, D. E., and Poeppel, E., editors (1995). Supercomputing in Brain Research: From Tomography to Neural Networks. World Scientific, Singapore.

Hertz, L., Schousboe, A., Boechler, N., Mukerji, S., and Fedoroff, S. (1978). Kinetic characteristics of the glutamate uptake into normal astrocytes in cultures. Neurochem. Res., 3:1-14.

Hessler, N. A., Shirke, A. M., and Malinow, R. (1993). The probability of transmitter release at a mammalian central synapse. Nature, 366:569-572.

Hille, B., editor (1992). Ionic Channels of Excitable Membranes (2nd ed.). Sinauer Associates, Sunderland.

Holmes, W. R. (1995). Modeling the effect of glutamate diffusion and uptake on NMDA and non-NMDA receptor saturation. Biophys. J., 69:1734-1747.

Huang, E. P. and Stevens, C. F. (1998). The matter of mind: molecular control of memory. Essays Biochem., 33:165-178.

Ichimura, T. and Hashimoto, P. H. (1988). Structural components in the synaptic cleft captured by freeze-substitution and deep etching of directly frozen cerebellar cortex. J. Neurocytol., 17:3-12.

Isaacson, J. S. and Nicoll, R. A. (1993). The uptake inhibitor L-trans-PDC enhances responses to glutamate but fails to alter the kinetics of excitatory synaptic currents in the hippocampus. J. Neurophysiol., 70:2187-2191.

Jahn, K., Bufler, J., and Franke, C. (1998). Kinetics of AMPA-type glutamate receptor channels in rat caudate-putamen neurones show a wide range of desensitization but distinct recovery characteristics. Eur. J. Neurosc., 10:664-72.

Jonas, P., Major, G., and Sakmann, B. (1993). Quantal components of unitary EPSCs at the mossy fiber synapse on CA3 pyramidal cells of rat hippocampus. J. Physiol. (Lond.), 472:615-663. 
Jones, M. V. and Westbrook, G. L. (1996). The impact of receptor desensitization on fast synaptic transmission. Trends Neurosc., 19:96-101.

Kaczmarek, L., Kossut, M., and Skangiel-Kramska, J. (1997). Glutamate receptors in cortical plasticity: molecular and cellular biology. Physiol. Rev., 77:217-255.

Kandel, E. R., Schwartz, J. H., and Jessel, T. M., editors (1996). Principles of neural science (3rd ed.). Elsevier, New York.

Kandel, E. R. and Spencer, W. A. (1968). Cellular and integrative properties of the hippocampal pyramidal cell and the comparative electrophysiology of cortical neurons. Int. J. Neurol., 6:266-296.

Katz, B. and Miledi, R. (1968). The role of calcium in neuromuscular facilitation. $J$. Physiol., 195:481-492.

Khanin, R., Parnas, H., and Segel, L. (1994). Diffusion cannot govern the discharge of neurotransmitter in fast synapses. Biophys. J., 67:966-972.

Kleinle, J., Vogt, K., Luescher, H. R., Mueller, L., Senn, W., Wyler, K., and Streit, J. (1996). Transmitter concentration profiles in the synaptic cleft: Analytical model of release and diffusion. Biophys. J., 71:2413-2426.

Klingauf, J. and Neher, E. (1997). Modeling buffered Ca2+ diffusion near the membrane: implications for secretion in neuroendocrine cells. Biophys. J., 72:674-690.

Koch, C. and Segev, I., editors (1998). Methods in Neuronal Modeling. From Ions to Networks (2nd ed.). MIT Press, Cambridge, Massachusetts.

Kruk, P. J., Korn, H., and Faber, D. S. (1997). The effect of geometrical parameters on synaptic transmission: A Monte Carlo simulation study. Biophys. J., 73:2874-2890.

Kullmann, D. A. and Asztely, F. (1998). Extrasynaptic glutamate spillover in the hippocampus: evidence and implications. Trends Neurosci., 21:8-14.

Kullmann, D. M., Min, M.-Y., F. Asztely, F., and Rusakov, D. A. (1999). Extracellular glutamate diffusion determines the occupancy of glutamate receptors at CA1 synapses in the hippocampus. Phil. Trans. R. Soc. Lond. B, 354:395-402.

Kullmann, D. M. and Nicoll, R. A. (1992). Long-term potentiation is associated with increases in quantal content and quantal size. Nature, 357:240-244.

Kusano, K. and Landau, E. M. (1975). Depression and recovery of transmission at the squid giant synapse. J. Physiol. (Lond.), 245:13-32.

Land, B. R., Harris, W. V., Salpeter, E. E., and Salpeter, M. M. (1984). Diffusion and binding constants for acetylcholine derived from the falling phase of miniature endplate currents. Proc. Natl. Adac. Sci. USA, 81:1594-1598. 
Land, B. R., Salpeter, E. E., and Salpeter, M. M. (1981). Kinetic parameters for acetylcholine interaction in intact neuromuscular junction. Proc. Natl. Adac. Sci. USA, 78:7200-7204.

Larson, J., Wong, D., and Lynch, G. (1986). Patterned stimulation at the theta frequency is optimal for the induction of hippocampal long-term potentiation. Brain. Res., 368:347-350.

Lenn, N. J. and Reese, T. S. (1966). The fine sturcture of nerve endings in the nucleus of the trapezoid body and the ventral cochlear nucleus. Am. J. of Anatomy, 118:375389.

Liley, A. W. and North, K. A. K. (1953). An electrical investigation of effects of repetitive stimulation on mammlian neuromuscular junction. J. Neurophysiol., 16:509-527.

Lim, R., Alvarez, F. J., and Walmsley, B. (1999). Quantal size is correlated with receptor cluster area at glycinergic synapses in the rat brainstem. J. Physiol. (Lond.), 516:505512.

Liu, G., Choi, S., and Tsien, R. W. (1999). Variability of neurotransmitter concentration and nonsaturation of postsynaptic AMPA receptors at synapses in hippocampal cultures and slices. Neuron, 22:395-409.

Liu, G. and Tsien, R. W. (1995). Properties of synaptic transmission at single hippocampal synaptic boutons. Nature, 375:404-408.

Llinas, R., Sugimori, M., and Silver, R. B. (1992). Microdomains of high calcium concentration in a presynaptic terminal. Science, 256:677-679.

Longworth, L. G. (1953). Diffusion measurement at $25^{\circ}$, of aqueous solutions of amino acids, peptides and sugars. J. Am. Chem. Soc., 75:5705-5709.

Mainen, Z. F., Malinow, R., and Svoboda, K. (1999). Synaptic calcium transients in single spines indicate that nmda receptors are not saturated. Nature, 399:151-155.

Malenka, R. C. and Nicoll, R. A. (1999). Long-term potentiation-a decade of progress? Science, 285:1870-1874.

Malinow, R. and Tsien, R. W. (1990). Presynaptic enhancement shown by whole-cell recordings of long-term potentiation in hippocampal slices. Nature, 346:177-180.

Marienhagen, J., Keller, B. U., and Zippelius, A. (1997). Kinetic model of excitatory synaptic transmission to cerebellar purkinje cells. J. Theor. Biol., 188:227-240.

Marienhagen, J. and Zippelius, A. (1995). Monte Carlo Simulation of a Structural Model for Long-term Potentiation, pages 243-248. In (Hermann et al., 1995). 
Markram, H., Pikus, D., Gupta, A., and Tsodyks, M. (1998a). Potential for multiple mechanisms, phenomena and algorithms for synaptic plasticity at single synapses. Neuropharmacology, 37:489-500.

Markram, H., Wang, Y., and Tsodyks, M. (1998b). Differential signaling via the same axon of neocortical pyramidal neurons. Proc. Natl. Acad. Sci. USA, 95:5323-5328.

Mennerick, S., Dhond, R. P., Benz, A., Xu, W., Rothstein, J. D., Danbolt, N. C., Isenberg, K. E., and Zorumski, C. F. (1998). Neuronal expression of the glutamate transporter glt-1 in hippocampal microcultures. J. Neurosc., 18:4490-4499.

Meyer, A. C. (1999). Untersuchungen zur Wahrscheinlichkeit der Transmitterfreisetzung an einer zentralen Synapse. Diplomarbeit. Max-Planck-Institut für biophysikalische Chemie, Göttingen.

Min, M.-Y., Rusakov, D. A., and Kullmann, D. M. (1998). Activation of AMPA, kainate, and metabotropic receptors at hippocampal mossy fiber synapses: Role of glutamate diffusion. Neuron, 21:561-570.

Murthy, V. N., Sejnowski, T. J., and Stevens, C. F. (1997). Heterogeneous release properties of visualized individual hippocampal synapses. Neuron, 18:599-612.

Naraghi, M. and Neher, E. (1997). Linearized buffered Ca2+ diffusion in microdomains and its implications for calculation of $[\mathrm{Ca} 2+]$ at the mouth of a calcium channel. $J$. Neurosci., 17:6961-6973.

Neher, E. (1986). Concentration profiles of intracellular calcium in the presence of a diffusible chelator. Exp. Brain Res., 14:80-96.

Neher, E. (1998a). Usefulness and limitations of linear approximations to the understanding of Ca++ signals. Cell Calcium, 24:345-357.

Neher, E. (1998b). Vesicle pools and $\mathrm{Ca}^{2+}$ microdomains: new tools for understanding their roles in neurotransmitter release. Neuron, 20:389-399.

Neher, E. (1999). personal communication.

Neher, E. and Augustine, G. J. (1992). Calcium gradients and buffers in bovine chromaffin cells. J. Physiol., 450:273-301.

Nicholson, C. and Phillips, J. M. (1981). Ion diffusion modified by tortuosity and volume fraction in the extracellular microenvironmet of the rat cerebellum. J. Physiol. (Lond.), 321:225-257.

Nusser, Z., Cull-Candy, S., and Farrant, M. (1997). Differences in synaptic GABA G $_{a}$ receptor number underlie variation in GABA mini amplitude. Neuron, 19:697-709. 
Oleskevich, S., Alvarez, F. J., and Walmsley, B. (1999). Glycinergic miniature synaptic currents and receptor cluster sizes differ between spinal cord interneurons. $J$. Neurophysiol., 82:312-319.

Otis, T. S., Kavanaugh, M. P., and Jahr, C. (1997). Postsynaptic glutamate transport at the climbing fiber-purkinje cell synapse. Science, 277:1515-1518.

Pavlidis, P. and Madison, D. V. (1999). Synaptic transmission in pair recordings from CA3 pyramidal cells in organotypic culture. J. Neurophysiol., 81:2787-2797.

Peters, A. and Kaiserman-Abramof, I. R. (1969). The small pyramidal neuron of the rat cerebral cortex: the synapses upon dendritic spines. Zeitsch. Zellforsch., 100:487-506.

Plesser, H. E. and Gerstner, W. (2000). Noise in integrate-and-fire neurons: From stochastic input to escape rates. Neural Comput., 12:367-384.

Press, W. P., Teukolsky, S. A., Vetterling, W. T., and Flannery, B. P., editors (1992). Numerical Recipes in C. The Art of Scientific Computing (2nd ed.). Cambridge University Press, Cambridge.

Quastel, D. M. (1997). The binomial model in flutuation analysis of quantal neurotransmitter release. Biophys. J., 72:728-753.

Rall, W. and Agmon-Snir, H. (1998). Cable Theory for Dendritic Neurons, pages 27-92. In (Koch and Segev, 1998).

Ravin, R., Parnas, H., Spira, M. E., Volfovsky, N., and Parnas, I. (1999). Simultaneous measurement of evoked release and $[\mathrm{Ca} 2+] \mathrm{i}$ in a crayfish release bouton reveals high affinity of release to Ca2+. J Neurophysiol, 81:634-642.

Rettig, J., Heinemann, C., Ashery, U., Sheng, Z. H., Yokoyama, C. T., Catterall, W. A., and Neher, E. (1997). Alteration of Ca2+ dependence of neurotransmitter release by disruption of Ca2+ channel/syntaxin interaction. J. Neurosci., 17:6647-6656.

Rice, M. E., Gerhardt, G. A., Hierl, P. M., Nagy, G., and Adams, R. N. (1985). Diffusion coefficients of neurotransmitters and their metabolites in brain extracellular fluid space. Neuroscience, 3:891-902.

Rieke, F., Warland, D., de Ruyter van Steveninick, R., and Bialek, W., editors (1997). Exploring the Neural Code. MIT Press, Cambridge, MA.

Rios, E. and Stern, M. D. (1997). Calcium in close quarters: microdomain feedback in excitation - contraction coupling and other cell biological phenomena. Ann. Rev. Biophys. Biomol. Struct., 26:47-82.

Roberts, W. M. (1994). Localization of calcium signals by a mobile calcium buffer in frog saccular hair cells. J. Neurosc., 14:3246-3262. 
Rosenmund, C., Clements, J. D., and Westbrook, G. L. (1993). Nonuniform probability of glutamate release at a hippocampal synapse. Science, 262:754-757.

Rosenmund, C. and Stevens, C. F. (1996). Definition of the readily releasable pool of vesicles at hippocampal synapses. Neuron, 16:1197-1207.

Rossi, D. J., Alford, S., Mugnaini, E., and Slater, N. T. (1995). Properties of transmission at a giant glutamatergic synapse in cerebellum: the mossy fiber-unipolar brush cell synapse. J. Neurophysiol., 74:24-72.

Rusakov, D. A. and Kullmann, D. M. (1998). Extrasynaptic glutamate diffusion in the hippocampus: ultrastructural constraints, uptake, and receptor activation. J. Neurosci., 18:3158-3170.

Sakmann, B. and Neher, E., editors (1995). Single-Channel Recording (2nd ed.). Plenum Press, New York.

Schikorski, T. and Stevens, C. F. (1997). Quantitative ultrastructural analysis of hippocampal excitatory synapses. J. Neurosc., 17:5858-5867.

Schneggenburger, R. (1999). unpublished.

Schneggenburger, R., Meyer, A. C., and Neher, E. (1999). Released fraction and total size of a pool of immediately available transmitter quanta at a calyx synapse. Neuron, 23:399-409.

Schneidman, E., Freedman, B., and Segev, I. (1998). Ion channel stochasticity may be critical in determining the reliability and precision of spike timing. Neural Comput., 10:1679-1703.

Shadlen, M. N. and Newsome, W. T. (1994). Noise, neural codes and cortical organization. Curr. Opin. Neurobiol., 4:569-579.

Sheng, Z., Retting, J., Cook, T., and Catterall, W. A. (1996). Calcium-dependent interaction of N-type calcium channels with the synaptic core complex. Nature, 379:451-454.

Sigworth, F. J. (1980). The variance of sodium current fluctuations at the node of ranvier. J. Physiol. (Lond.), 307:97-129.

Silver, R. A., Cull-Candy, S. G., and Takahashi, T. (1996). Non-NMDA glutamate receptor occupancy and open probability at a rat cerebellar synapse with single and multiple release sites. J. Physiol. (Lond.), 494:231-250.

Silver, R. A., Cull-Candy, S. G., and Takahashi, T. (1998). Locus of frequency-dependent depression identified with multiple-probability fluctuation analysis at rat climbing fibre-purkinje cell synapses. J. Physiol. (Lond.), 510:881-902. 
Simon, S. M. and Llinas, R. R. (1985). Compartmentalization of the submembrane calcium activity during clacium influx and its significance in transmitter release. Biophys. J., 48:485-498.

Sorra, K. E. and Harris, K. M. (1993). Occurrence and three-dimensional structure of multiple synapses between individual radiatum axons and their target pyramidal cells in hippocampal area CA1. J. Neurosc., 13:3736-3748.

Spruston, N., Jonas, P., and Sakmann, B. (1995). Dendritic glutamate receptor channels in rat hippocampal CA3 and CA1 pyramidal neurons. J. Physiol. (Lond.), 482:325352.

Stern, M. D. (1992). Buffering of calcium in the vicinity of a channel pore. Cell Calcium, 13:183-192.

Stevens, C. F. and Tsujimoto, T. (1995). Estimates for the pool size of releasable quanta at a single central synapse and for the time required to refill the pool. Proc. Natl. Acad. Sci. USA, 92:846-849.

Stevens, C. F. and Wesseling, J. F. (1998). Activity-dependent modulation of the rate at which synaptic vesicles become available to undergo exocytosis. Neuron, 20:12431253 .

Stiles, J. R., van Helden, D., Bartol, T. M., Salpeter, E. E., and Salpeter, M. M. (1996). Miniature endplate current rise time $<100 \mathrm{~ms}$ from improved dual recordings can be modeled with passive acetylcholine diffusion from a synaptic vesicle. Proc. Natl. Acad. Sci. USA, 93:5747-5752.

Suzuki, S. S. and Smith, G. K. (1985). Burst characteristics of hippocampal complex spike cells in the awake rat. Exp. Neurol., 89:90-95.

Takahashi, A., Camacho, P., Lechleiter, J. D., and Herman, B. (1999). Measurement of intracellular calcium. Physiol. Rev., 79:1089-125.

Takahashi, M., Sarantis, M., and Atwell, D. (1996). Postsynaptic glutamate uptake in rat cerebellar purkinje cells. J. Physiol. (Lond.), 497:525-530.

Tang, C. M., Margulis, M., Shi, Q. Y., and Fielding, A. (1994). Saturation of postsynaptic glutamate receptors after quantal release of transmitter. Neuron, 13:1385-1393.

Thomson, A. M. and Deuchars, J. (1994). Temporal and spatial properties of local circuits in neocortex. Trends Neurosci., 17:119-126.

Titz, S. and Keller, B. (1996). Functional diversity of excitatory synaptic currents mediated by long and short residence times of glutamate in the synaptic cleft. Biophys. J., 70:A 372 . 
Titz, S. and Keller, B. (1997). Rapidly deactivating AMPA receptors determine excitatory synaptic transmission to interneurons in the nucleus tractus solitarius from rat. $J$. Neurophysiol., 78:82-91.

Tong, G. and Jahr, C. E. (1994). Block of glutamate transporters potentiates postsynaptic excitation. Neuron, 13:1195-1203.

Trommershäuser, J., Marienhagen, J., and Zippelius, A. (1999). Stochastic model of central synapses: slow diffusion of transmitter interacting with spatially distributed receptors and transporters. J. Theor. Biol., 198:101-119.

Trommershäuser, J., Parthasarathy, H., Edwards, F. A., and Zippelius, A. (1997). Stochastic models of the synaptic transmission in the CNS: importance of the diffusion coefficient and the receptor distribution. Soc. of Neurosc. Abstr., 23:658.

Tsodyks, M. V. and Markram, H. (1997). The neural code between neocortical pyramidal neurons depends on neurotransmitter release probability. Proc. Natl. Acad. Sci. USA, 94:719-723.

Tsodyks, M. V., Pawelzik, K., and Markram, H. (1998). Neural networks with dynamic synapses. Neural Comput., 10:821-835.

Uteshev, V. V. and Pennefather, P. S. (1996a). A mathematical description of mpsc generation at CNS synapses. Biophys. J., 71:1256-1266.

Uteshev, V. V. and Pennefather, P. S. (1996b). Phasic inhibition and state-dependent inhibition: an explicit solution for a three-state ion channel system. J. Theor. Biol., 181:11-23

Uteshev, V. V. and Pennefather, P. S. (1997). Analytical description of the activation of multi-state receptors by continuous neurotransmitter signals at brain synapses. Biophys. J., 72:1127-1134.

Varela, J. A., Sen, K., Gibson, J., Fost, J., Abbott, L. F., and Nelson, S. B. (1997). A quantitative description of short-term plasticity at excitatory synapses in layer $2 / 3$ of rat primary visual cortex. J. Neurosci., 17:7926-7940.

von Gersdorff, H., Schneggenburger, R., Weis, S., and Neher, E. (1997). Presynaptic depression at a calyx synapse: the small contribution of metabotropic glutamate receptors. J. Neurosci., 17:8137-8146.

Wahl, L. M., Pouzat, C., and Stratford, K. J. (1996). Monte Carlo simulation of fast excitatory synaptic transmission at a hippocampal synapse. J. Neurophysiol., 75:597608.

Walmsley, B., Alvarez, F. J., and Fyffe, R. E. W. (1998). Diversity of structure and function at mammalian central synapses. Trends Neurosc., 21:81-88. 
Wang, G. J., Chung, H. J., Schnuer, J., Pratt, K., Zable, A. C., Kavanaugh, M. P., and Rosenberg, P. A. (1998). High affinity glutamate transport in rat cortical neurons in culture. Mol. Pharmacol., 53:88-96.

Wang, L. Y. and Kaczmarek, L. K. (1998). High-frequency firing helps replenish the readily releasable pool of synaptic vesicles. Nature, 394:384-388.

Weis, S., Schneggenburger, R., and Neher, E. (1999). Properties of a model of $\mathrm{Ca}^{++}$ dependent vesicle pool dynamics and short term synaptic depression. Biophys J., $77: 2418-2429$.

Wu, L. G. and Borst, J. G. (1999). The reduced release probability of releasable vesicles during recovery from short-term synaptic depression. Neuron, 23:821-832.

Wu, L. G., Westenbroek, R. E., Borst, J. G. G., Catterall, W. A., and Sakmann, B. (1999). Calcium channel types with distinct presynaptic localization couple differentially to transmitter release in single calyx-type synapses. J. Neurosc., 19:726-736.

Xu, T., Rammner, B., Margittai, M., Artalejo, A. R., Neher, E., and Jahn, R. (1999). Inhibition of snare complex assembly differentially affects kinetic components of exocytosis. Cell, 99:713-722.

Yamada, W. M. and Zucker, R. S. (1992). Time course of transmitter release calculated from simulations of a calcium diffusion model. Biophys. J, 73:532-545.

Zador, A. M. and Dobrunz, L. E. (1997). Dynamic synapses in the cortex. Neuron, 19:1-4.

Zucker, R. S. (1989). Short-term synaptic plasticity. Annu. Rev. Neurosci., 12:13-31.

Zucker, R. S. (1996). Exocytosis: A molecular and physiological prespective. Neuron, 17:1049-1055.

Zucker, R. S. (1999). Calcium- and activity-dependent synaptic plasticity. Curr. Opin. Neurobiol., 9:305-313. 


\section{Acknowledgement}

First of all, I wish to thank my advisor Prof. Annette Zippelius for letting me work on this subject and for her constant encouragement over the last years. Her clear and determined approach to open problems has always served as a shining example for me. Throughout the whole time of my thesis she has been supporting me in any possible way.

I am deeply indebted to Dr. Frances Edwards for introducing me to the big wide zoo of neuroscience, inviting me to London and dragging me all the way to New Orleans. She has been a great help in all sorts of situations, always passionately reassuring me that there indeed are questions to be addressed by theoreticians.

My thesis would not have been possible without experimental input. I was lucky enough to learn from experiments done in the group of Prof. Erwin Neher. I wish to thank him for giving me the opportunity to collaborate with him and his group and for reading parts of the manuscript. It has been a pleasure to work with Dr. Ralf Schneggenburger. I am most grateful for his immense patience concerning all my questions about the "real" physiology, for his energy to keep the project going, and for reading large parts of my manuscript. Also thanks to Dr. Jürgen Klingauf, Alexander Meyer, and Volker Scheuss for helpful discussions.

I wish to thank Dr. Bernhard Keller for providing a hot-line for all kinds of queries, giving me the chance to spend time in his lab and witnessing the transformation of a living animal into a brain-slice. I thank Bodo Vanselow for simultaneously coping with me and the experimental setup.

I am most grateful to Prof. George Hess for letting me stay in his lab at Cornell in 1997, giving me an idea about the world of chemistry, teaching me to keep things simple and for a wonderful time in Ithaca. I wish to thank Susan Coombs and all the other group members for giving me a warm welcome. A very special thanks to Dr. Heyi Li for introducing me to the Caenorhabditis elegans and the rules of American TV sports and to Dr. Vasanthi Jayaraman for an enjoyable collaboration, entertaining "candidate dinners", and for keeping in touch.

Within these last four years I have been extremely lucky with my "scientific home" here in the Villa. I have profited from many discussions with Prof. Reiner Kree. His inspiring enthusiasm and curiosity for biological topics has served as a constant source of motivation. I thank Dr. Kurt Broderix for helping me with several problems on stochastic processes and sitting through the worst movie ever. (Terribly missing you....) I wish to thank Dr. Peter Müller for his trust and attention, biking-trips in the rain, and for taking the lead. It has been a great pleasure to share my office with Timo Aspelmeier, Dr. Martin Huthmann, and Henning Löwe. Thanks to Martin for stories on nearly everything, to Timo for shaking the head about my ideas, but always helping to put them into practice, and to Henning for voluntarily entering our small world on the third floor. Also all the other group members have made these past years, in particular our joint time among monks, more than pleasant - not to forget Gisela Mesecke, whose open heart and caring 
attention has always substantially contributed to this very special surrounding.

I have profited a lot from discussions with Prof. David Colquhoun, Prof. John Guggenheimer, Prof. Julian Jack, Dr. Martin Stetter, and Klaus Stiefel.

I thank Prof. Theo Geisel for reviewing my thesis and Dr. Thomas Heimburg and Prof. Günther Schmahl for serving on my committee.

I wish to thank the Graduiertenkolleg Organisation und Dynamik neuronaler Netze for supporting my work with a scholarship and the DAAD for granting me a scholarship (Doktorandenstipendium HSP II/AUFE program) to finance my stay at Cornell.

I am most grateful to Dr. Hemai Parthasarathy for creating a joint poster, driving all the way to Philadelphia, and making me meet Tibby; Rufin VanRullen for the leash, a drawing, and being stuck in Venice; Jan-Alexander Heimel for his enciphered messages; Dr. Herve Isambert and Dr. Joachim Jacobson for trips on different continents; Dr. Badrinarayan Krishnamachari for the nightly visit of all my region's castles, Dr. Anoop Prasad for always providing more than a shelter in Brooklyn; Dr. Sunil Saxena and Dr. Manish Tandon for the hilarious six months under the same roof and a Salsa class with probably the highest number of Indian participants ever.

I thank Thilo Wagner for being the reliable and stubborn friend he is; Nicole Mamier for tons of cookies, exchange on family matters, and all kind of night-life; Dr. Uta Bongenberg for her openness and making me stand on my hands and in front of little girls again; Ursula Hübner for giving a home to my two ponies; Michaela Rössler for so much energy and curiosity; Parascevi Anagnostopoulou-Bock for her warmheartedness and fabulous Greek food; Frank Zander for lifting, listening, and the lottery-ticket; and the rest of the gang, Christoph Hannig, Dr. Wiebke Lohstroh, Helge Wenzel, and Arnd Zander for more than just company on Tuesday-evenings.

I thank Dr. Kamal Bhattacharya, not only for fighting his way through the manuscript, being a great help on numerous computational problems, and enduring repeated reports on stuck projects. He has made my world so much larger - not just eastwards and westwards - and has always been believing that I am going somewhere, even when I had lost track.

As the first physicist in my family I have always been enjoying the disbelieving astonishment about stories of my work. I wish to thank my parents Siegrid and Rolf Trommershäuser for their loving support and persistent efforts to remain part of my life. I would have been lost many times without my mother's pride and will and my father's integrity, humor and ability to face the inevitable. I thank my sister Tatjana Trommershäuser for her friendship, calling me a monkey in front of hundreds of people, and adding something very special to my life. I am most grateful to my grandmother Emma Krampitz for always being there for me. 


\section{Curriculum Vitae}

Born: 28.02.1971 in Frankfurt am Main, Germany

Parents: Rolf and Siegrid Trommershäuser

\section{Studies and Training}

$04 / 2000$

$10 / 1995$

$02 / 1995$

$09 / 1991$

$05 / 1989$

\section{Awards}

$01 / 1996-04 / 1999$

$03 / 1997-09 / 1997$

08/1999-09/1999
PhD in Physics

Georg-August University Göttingen

Vordiplom in Psychology

Georg-August University Göttingen.

Diplom in Physics

Georg-August University Göttingen.

Vordiplom in Physics

Justus-Liebig University Gießen.

Abitur

Gymnasium Philippinum, Weilburg.

Fellowship of the Graduiertenkolleg

"Organization and dynamics of neural networks"

Georg-August University Göttingen

DAAD-Doktorandenstipendium

Cornell University, Ithaca, NY, U.S.A.

EU-course in Computational Neuroscience

ICTP, Trieste, Italy 


\section{List of publications}

\section{J. TrommershäUser AND A. Zippelius}

Biophysical model of a single synaptic connection: transmission properties are determined by the cooperation of pre- and postsynaptic mechanisms

to appear in the Proc. of CNS 2000, 2000.

2. J. Trommershäuser, S. Titz, B. U. Keller And A. Zippelius

Variability of Excitatory Currents due to Single Channel Noise, Receptor Number and Morphological Heterogeneity

submitted.

3. J. Trommershäuser, J. Marienhagen And A. Zippelius

Stochastic model of central synapses: slow diffusion of transmitter interacting with spatially distributed receptors and transporters

J. Theor. Biol. 198, 101, 1999.

4. J. Trommershäuser, S. Titz, B.U. Keller And A. Zippelius

Monte Carlo simulations of excitatory synaptic signals in central neurons: EPSC fluctuations as a function of receptor number and residence time of glutamate in the synaptic cleft

Biophys. J. 76, A 53, 1999.

5. J. TrommershäUser AND A. Zippelius

Studying the effect of spatially distributed receptors for a limited number of receptors Europ. J. Neurosc. 10, 171, 1998.

6. J. TommershäUser And B. U. Keller And A. Zippelius

Monte Carlo simulations of excitatory synaptic currents in interneurons of the rat cerebellum and brain stem

In: New Neuroethology on the Move: proceedings of the 26th Göttingen Neurobiology Conference 1998

Eds.: N. Elsner and R. Wehner

Stuttgart; New York: Thieme; p. 600, 1998.

7. J. Trommershäuser, H. Parthasarathy, F. A. Edwards and A. Zippelius Stochastic models of the synaptic transmission in the CNS: importance of the diffusion coefficient and the receptor distribution

Soc. of Neurosc. Abstr. 23, 658, 1997.

8. J. Marienhagen, J. Tommershäuser And A. Zippelius

Monte Carlo simulation of a structural model for long-term potentiation

In: Brain and Evolution: proceedings of the 24th Göttingen Neurobiology Conference 1996

Eds.: N. Elsner and R. Wehner

Stuttgart; New York: Thieme; p. 577, 1996. 